Genetic fingerprints of microalgal culture strains: amplified fragment length polymorphism (AFLP)

for investigations below the species level

\author{
Dissertation \\ zur Erlangung des Doktorgrades \\ der Mathematisch-Naturwissenschaftlichen Fakultäten \\ der Georg-August-Universität zu Göttingen
}

vorgelegt von

Julia Müller

aus Northeim

Göttingen 2005 
D7

Referent: Prof. Dr. Thomas Friedl Korreferent: Prof. Dr. Ivo Feußner

Tag der mündlichen Prüfung: 28.06.2005 


\section{Contents}

Abbreviations of culture collections of algae ................................................................................. 1

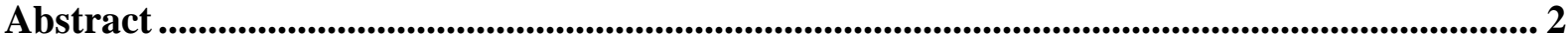

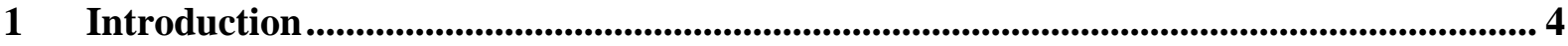

1.1 Importance of genetic analyses below the species level in microalgae......................4

1.2 Genetic analyses below the species level as an objective of service culture collections

1.3 Genetic analyses below the species level for the discrimination of multiple and duplicate strains

1.4 Genetic analyses below the species level to assess the genetic integrity of cryopreserved microalgae.

1.5 Amplified fragment length polymorphism ..................................................... 7

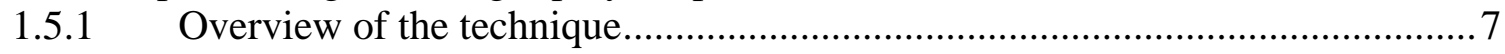

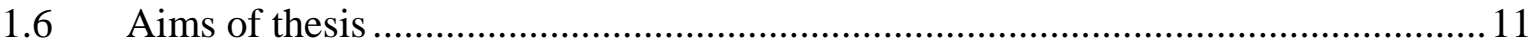

2 Distinction of isolates among multiple strains of Chlorella vulgaris and testing conspecificity with amplified fragment length polymorphism and ITS rDNA sequences..... 12

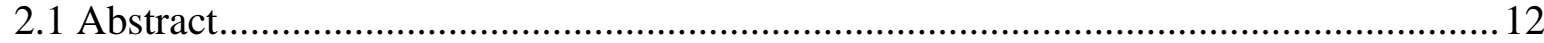

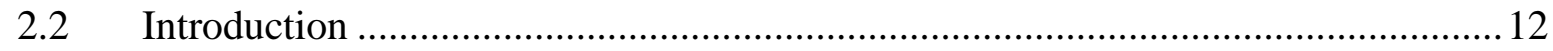

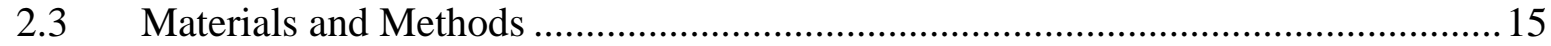

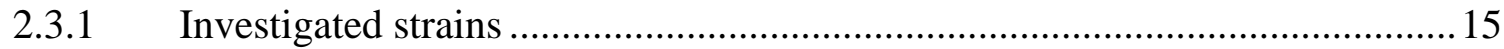

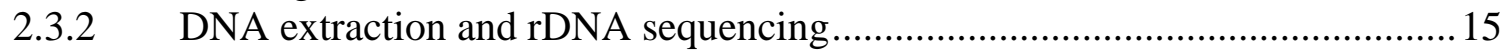

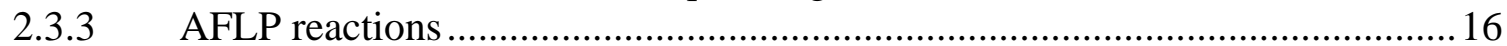

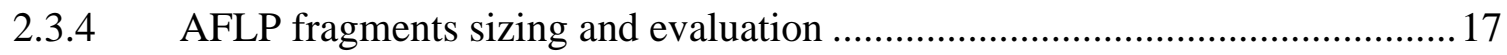

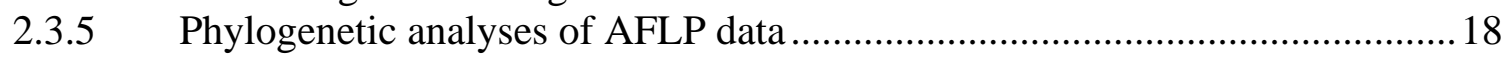

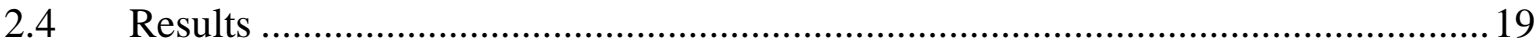

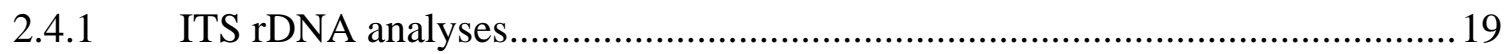

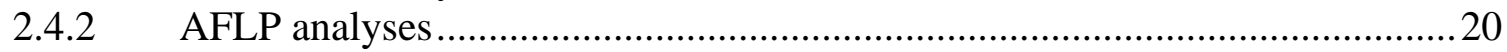

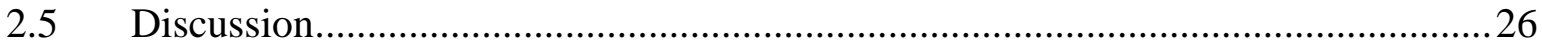

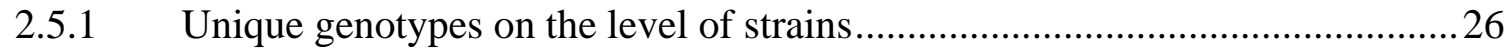

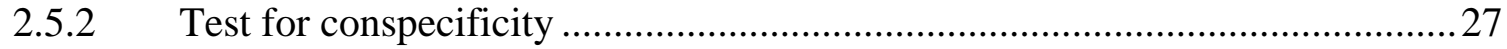

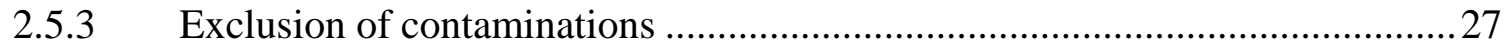

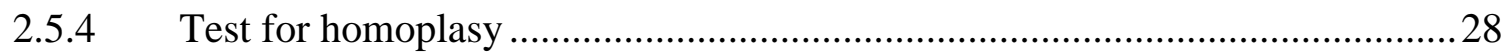

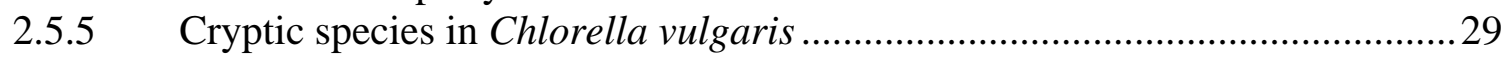

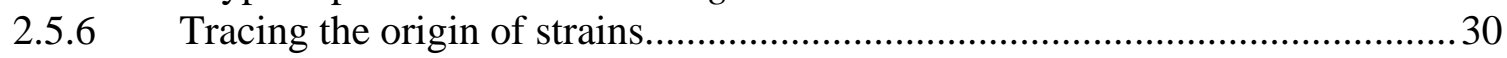

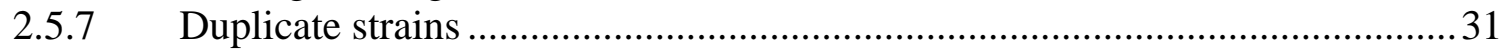

2.5.8 Evaluation and reproducibility of AFLP patterns........................................ 31

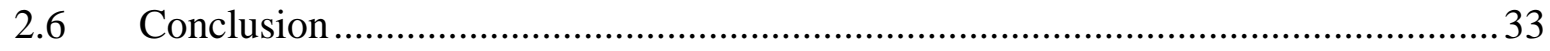

3 Detection of genetic alterations among pigment mutants of Parachlorella kessleri induced by different mutagenesis techniques (UV light, $x$-ray, radioisotope) based on amplified fragment length polymorphism (AFLP) ........................................................................... 34

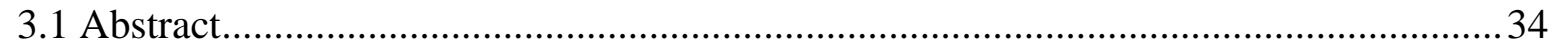

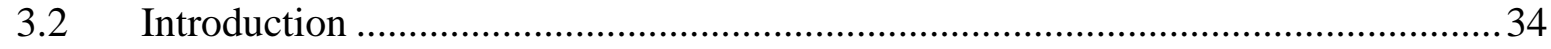

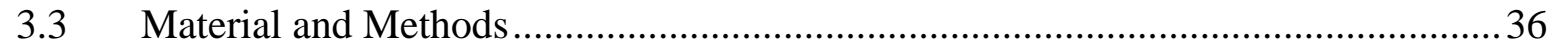

3.3.1 Investigated strains, DNA extraction, AFLP and 18S rDNA analyses ............36

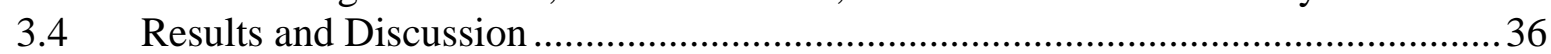

3.4.1 Genetic differences between wildtype and mutants of $P$. kessleri ....................36

3.4.2 Test for reproducibility of the banding patterns ............................................. 38 


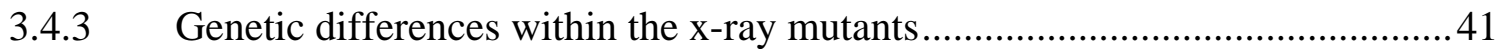

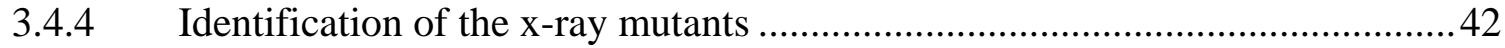

4 Applicability of amplified fragment length polymorphism for genetic characterization of non-axenic strains of microalgae: a case study using mutants of Dunaliella salina........... 44

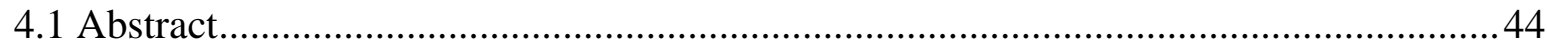

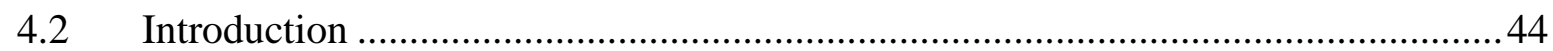

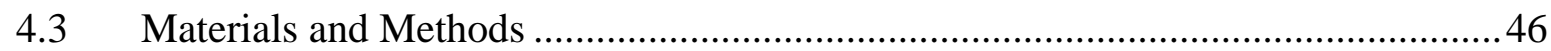

4.3.1 Investigated strains and sample preparation...............................................46

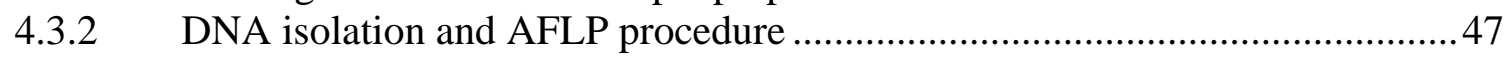

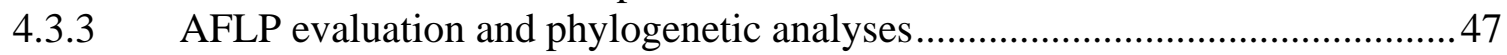

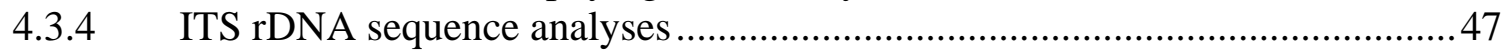

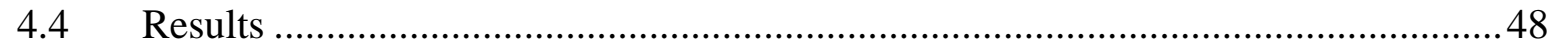

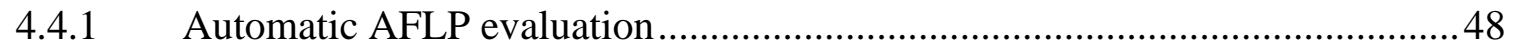

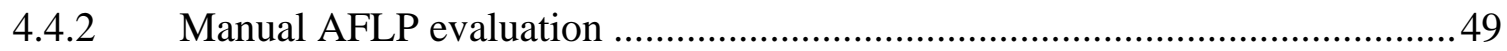

4.4.3 Comparison of banding patterns from washed and unwashed cultures.............49

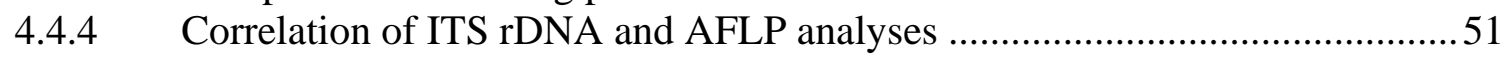

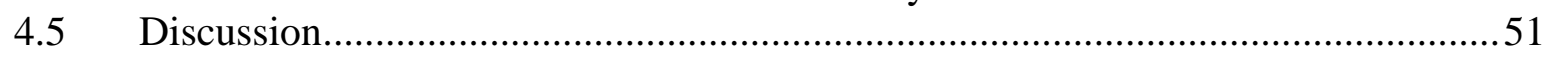

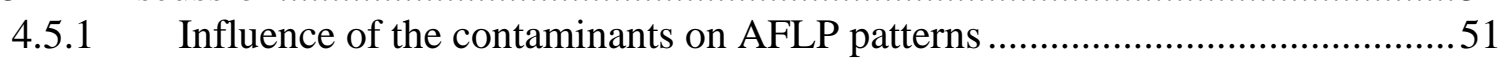

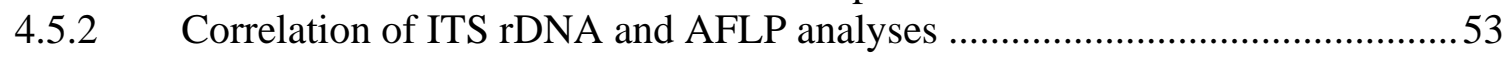

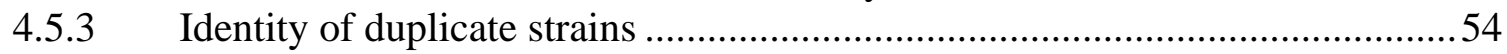

4.5.4 Comparison of the wildtype and the mutants ............................................... 55

4.5.5 Comparison of automatic and manual evaluation ...........................................56

5 Amplified fragment length polymorphism (AFLP) in genetic stability tests of cryopreserved microalgae in combination with reproducibility testing of AFLP banding

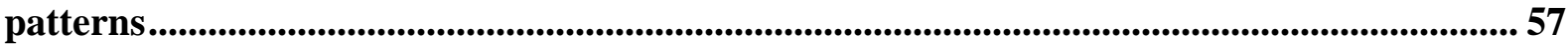

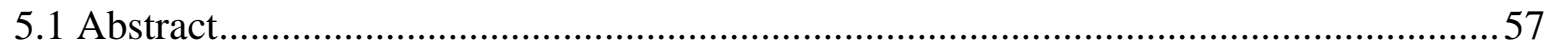

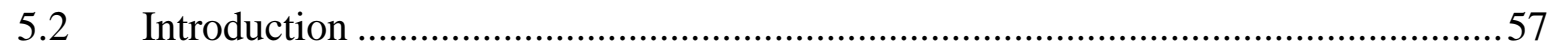

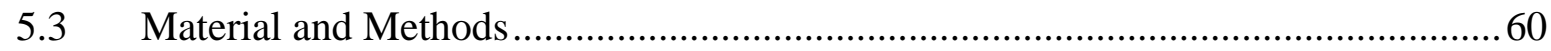

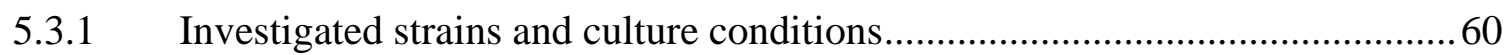

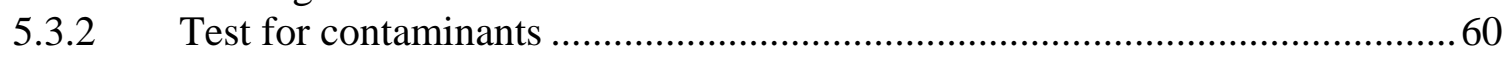

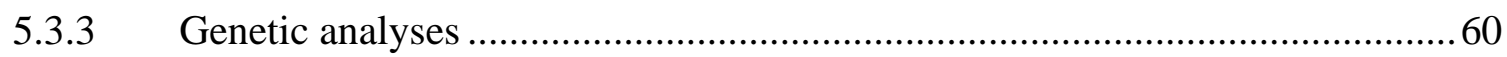

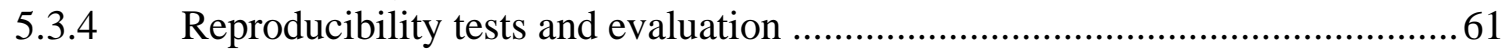

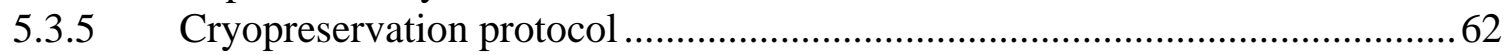

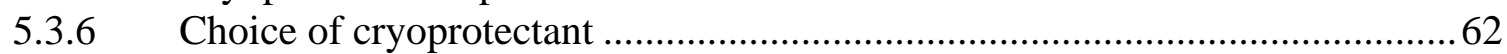

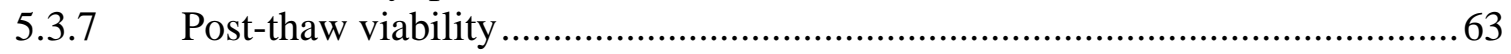

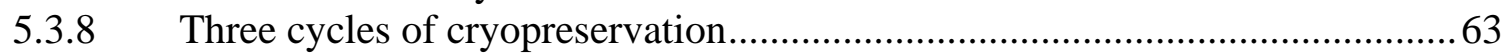

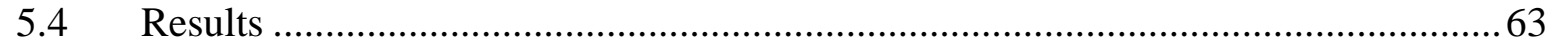

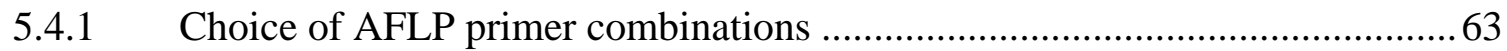

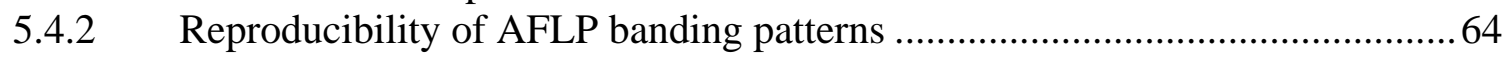

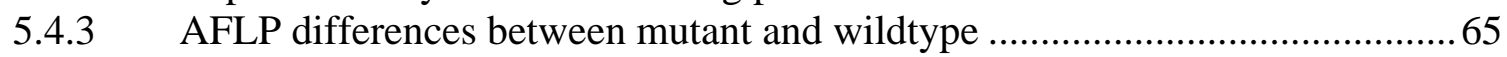

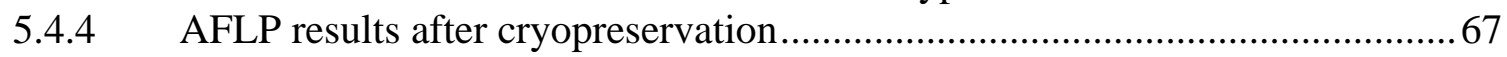

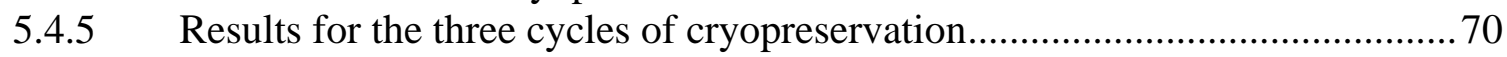

5.4.6 Comparison of genetic analyses and PTV levels .......................................... 70

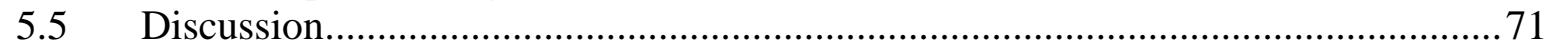

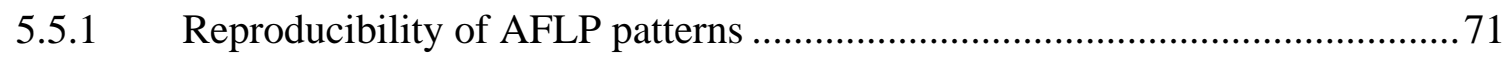

5.5.2 Reasons for non-reproducible fragments..................................................... 71

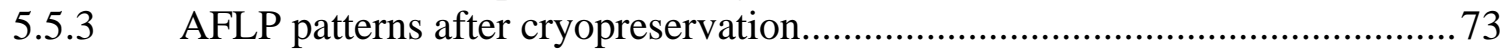

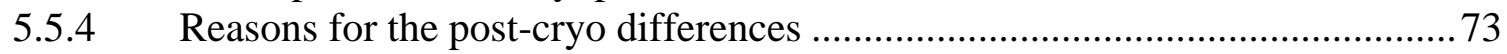

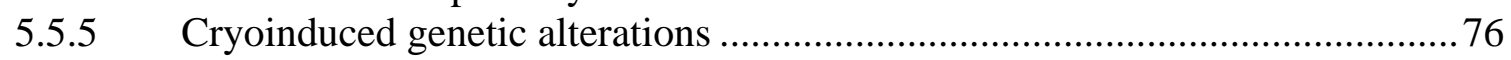


5.6 Conclusions with respect to the differences obtained after cryopreservation ...........77

5.7 AFLP reproducibility tests between European laboratories .....................................79

6 Detection of a viral infection in Chlorella and analysis of host ranges using amplified

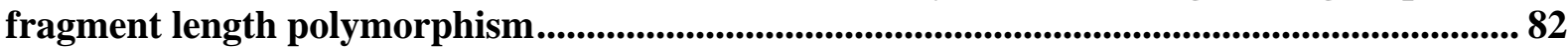

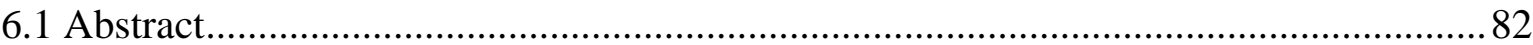

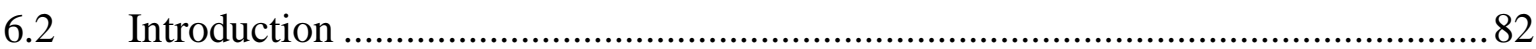

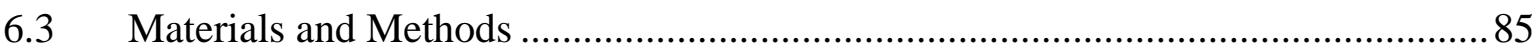

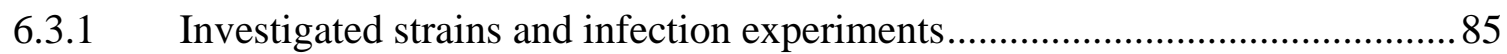

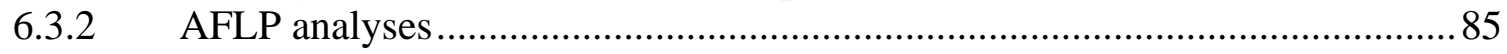

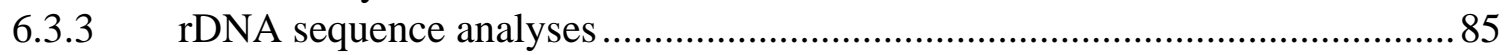

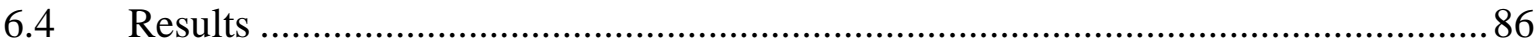

6.4.1 AFLP analyses of pure and infected Chlorella cultures..................................86

6.4.2 Results of rDNA- and AFLP analyses and the infection experiment with the

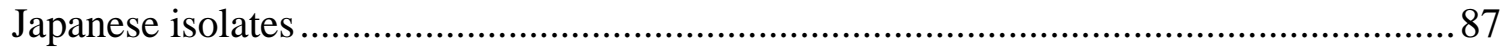

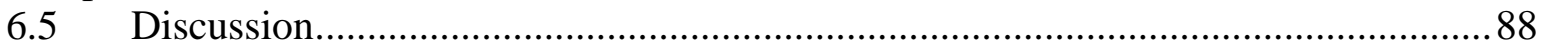

6.5.1 Detection of viral contaminations of algal strains using AFLP.......................8

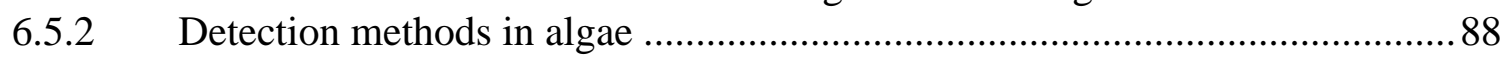

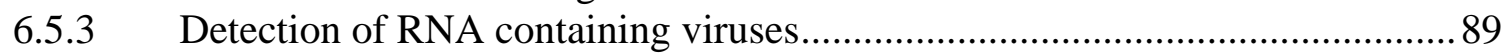

6.5.4 AFLP patterns show degradation of host DNA............................................... 89

6.5.5 Comparison of American and Japanese isolates to assess the host range of

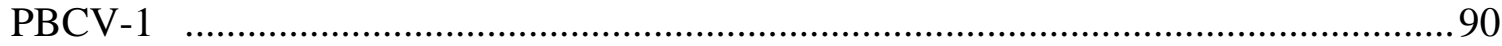

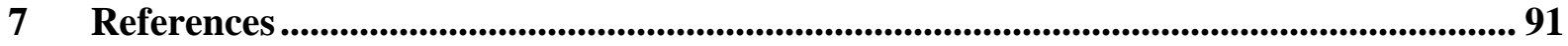

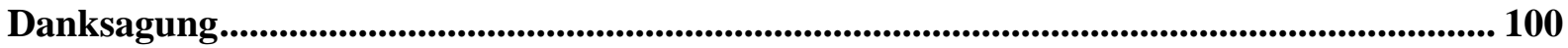

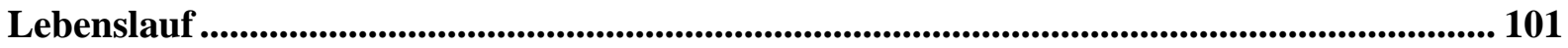




\section{Abbreviations of culture collections of algae}

SAG Culture Collection of Algae at Göttingen University, Göttingen, Germany

CCAP Culture Collection of Algae and Protozoa, Dunbeg, United Kingdom

CCALA Culture Collection of Algal Laboratory, Trebon, Czech Republic

UTEX

Culture Collection of Algae at the University of Texas, Austin, USA

ATCC American Type Culture Collection, Manassas, VA, USA

DCCBC

Dunaliella Culture Collection at Brooklyn College, New York, USA

NIES Microbial Culture Collection, National Institute for Environmental Studies, Tsukuba, Japan

IAM Institute of Molecular and Cellular Biosciences, University of Tokyo, Tokyo, Japan 


\begin{abstract}
The aim of the present thesis was to analyse microalgal culture strains at the level below species. This was important to improve the abilities of service culture collections of algae to preserve biodiversity more efficiently and to provide their user community with correctly identified and clean organisms. As examples commonly used microalgal strains of great value in applied research were investigated with genetic fngerprints provided by the AFLP method.
\end{abstract}

Genetic diversity among multiple strains of the same species was revealed for the green alga Chlorella vulgaris. AFLPs distinguished unique genotypes, revealed genetic signatures for each isolate and identified the presence of cryptic species. ITS rDNA sequences were required to check for conspecificity in comparison with authentic culture material. No genomic differences could be detected among duplicate strains that were maintained in different culture collections. Within the species, strains representing different isolates were genetically clearly different. This finding advocates the need to carefully record which strain has been used in any experiment, because the detected genetic differences may be correlated with phenotypic differences.

Further studies were performed to assess the discrimination power of AFLP. At an even finer level, AFLP discriminated pigment mutants of Parachlorella kessleri. Clear differences were found between and among strains mutated by UV light and radioisotope whereas no genetic differences were found between x-ray-induced mutants. The latter were identified as a different species.

In AFLP analyses of cultures that are contaminated it is uncertain whether a particular fragment is derived from the ogranism in study or the contamination. Many microalgae, e.g. Dunaliella salina, are difficult to purify. Reliable results were obtained for mutants of $D$. salina when AFLP patterns from cultures with different alga/contaminant ratios where compared among each other and with the pattern of the separated corresponding contamination. Viral infections that are otherwise difficult to detect were studied with AFLP in Chlorella sp.. In the studied lytic system, viral fragment patterns could be clearly distinguished from those of the algae. Even a distinction of isolates within the host range of the same virus was achieved with AFLP.

AFLP patterns were evaluated for phylogenetic analyses fully automated, but manual refinement was needed for comparisons of rather similar patterns. Replications of AFLPs (three times including two independent DNA extractions) minimized the amount of nonreproducible fragments. This was tested on a broad range of microalgae of different morphologies and taxonomic positions. 
Mislabeling of culture strains was identified as a major problem of traditional perpetual maintenance, i.e. it was found here whenever more than one strain of a certain species was investigated. Cryopreservation which minimizes handling errors as well as the risk of contaminations appears as an appropriate alternative. The genetic integrity of cryopreserved microalgae that differed in their sensitivity towards cryopreservation procedures was assessed with AFLP. Differences were obtained after cryopreservation for some samples of cyanobacteria and eukaryotic microalgae of different post-thaw viability levels. Possible reasons for these differences included e.g. changes in DNA methylation, cryoselection of subpopulations, and cryo-induced mutations. 


\section{Introduction}

\subsection{Importance of genetic analyses below the species level in microalgae}

The lack of morphological characters makes the delimitation of species difficult for most microalgae (e.g. Fig. 1.3a and b). To circumvent this disadvantage, biochemical and physiological characters have been used to help to unravel microalgal taxonomy. However, these characters reach their resolution limit at the species level. Molecular markers provided a new type of data that could be used to test hypotheses concerning the classification of microalgae based on morphological, physiological and biochemical characters. Today, molecular markers are standard techniques for microalgal taxonomy. By employing molecular markers, a number of polyphyletic genera have been identified (Chlorella, e.g. Huss et al. 1999, Chlamydomonas e.g. Pröschold et al. 2001 or Planophila Friedl and O’Kelly 2002), and the existence of genetically distinct lineages within single morpho-species has been revealed. The latter are often interpreted as cryptic phylogenetic species (e.g. Taylor et al. 2000, Lewis and Flechtner 2004). These findings demonstrate that considerable genetic diversity remains to be explored even at the lowest levels of microalgal taxonomy, namely below that of the species. Sensitive molecular methods are therefore required to unravel genomic variation within strains of a single species. However, for this purpose only a few molecular marker techniques are available and most of them have limitations. Either the molecular marker cannot be easily used for a wide range of microalgae without prior knowledge of the genome (microsatellites or simple sequence repeats, DeBruin et al., 2003; Iglesias-Rodriguez et al. 2002), or it is known to be non-reproducible between laboratories (random amplified polymorphic DNA, e.g. Penner et al. 1993). Sequence analyses of the internal transcribed spacer (ITS) region of the ribosomal DNA are commonly used for phylogenetic inference at the generic and intrageneric levels (Álvarez and Wendel 2003) and have been applied to a broad range of algae (e.g. Coleman and Mai 1997, Behnke et al. 2004). The usefulness of ITS rDNA analyses for investigations of microalgae below the species level is problematic, because this marker may reach its limits at the level of species (e.g. John et al. 2004) and may be not appropriate for cyanobacteria (e.g. Boyer et al. 2001).

One method that is not affected by the drawbacks described here for other molecular marker techniques is the fingerprinting technique Amplified Fragment Length Polymorphism (AFLP, Vos et al. 1995, Fig. 1.1). This method has been frequently used in higher plant 
taxonomy, often in combination with sequence analyses of marker molecules. In algae, AFLP has been much less used, i.e. to resolve genetic distances among geographic isolates and to delineate species of marine macroalgae (e.g. Schaeffer et al. 2002, Murphy and Schaffelke 2003, Erting et al. 2004), for marine microalgae (De Bruin et al. 2004, John et al. 2004) or charophytes (Mannschreck et al. 2002). In addition, AFLPs have proven to be reliable tools in bacterial taxonomy (Arias et al. 2004, Burke et al. 2004) both for the delineation of species (identification) and for the discrimination of strains ('typing', Savelkoul et al. 1999). However, AFLP has been applied to freshwater microalgae only once (Werner et al. 2001). The objective of the present study was to test the suitability of this technique for different applications on a wider range of microalgae. All of these applications draw directly from problems and questions related to culture strains of microalgae.

\subsection{Genetic analyses below the species level as an objective of service culture collections}

Cyanobacteria and eukaryotic microalgae are employed for a wide spectrum of uses, ranging from their application as model organisms for plant physiology and biochemistry to a number of biotechnological applications (Day et al. 1999). Microalgae play a role in numerous economically important products, e.g. health food, aquaculture feed, pigments, vitamins, antioxidants or antimicrobial agents. Such diverse applications require physiologically and genetically stable cultures as well as correctly identified organisms to guarantee reproducibility and reliability. One of the main objectives of service culture collections of microalgae is the fulfillment of these requirements. In addition culture collections provide authentic specimens for research, education, training, bioassay use or use as aquaculture starter cultures (Day et al. 1999). One of the main goals is therefore to obtain genetic signatures that allow the discrimination of various isolates of the same algal species. The latter is also important for culture collections when accessioning a new strain in order to avoid excessive replication in their holdings. 


\subsection{Genetic analyses below the species level for the discrimination of multiple and duplicate strains}

In public culture collections the same species of microscopic alga is often represented by different isolates that are referred to as 'multiple strains'. This constitutes a serious concern for the culture collections, because it could mean that a considerable proportion of their holdings of living algae may be identical replicates. For example, at the SAG culture collection there are 360 strains (16\% of its total holdings) that correspond to just 49 species of which each is represented by five or more strains. 'Multiple strains' may represent isolates of different origins, i.e. they were isolated from geographically distant localities and different habitats and, therefore, genomic diversity can be expected among these 'multiple strains'. The amount of genetic redundancy (i.e. identical replicates) among such multiple strains is not known. The question if these strains represent genetic diversity or redundancy is relevant for the users of the collection as well as for the collection itself. In addition, the same isolate may be maintained at different culture collections; such strains are referred to as 'duplicate strains' here. An example are the strains that have been isolated by E. G. Pringsheim (1881-1970) that are maintained in parallel at at least four different service culture collections since many decades (Day et al. 2004). Genetic diversity between 'duplicate strains' could have been introduced 'artificially', i.e. by different maintenance methods and culture regimes at different collections. Continuous subculturing of an algal strain over thousands of generations under the selective pressure of laboratory culture is likely to favor genetic changes due to selective pressure and/or genetic drift (Brand and Diller 2004). Also, over many decades human error may result in a higher likelihood of mislabeling or a 'mix up' on performing continuous subculturing (Lorenz et al. 2005). Strains of the same isolate may have been stored at an ultralow temperature (cryopreservation, Karlsson and Toner 1996) in one culture collection while they were also maintained in actively metabolizing state in another. This may lead to duplicates of the same clone at two culture collections being genetically different.

An investigation of multiple and duplicate strains at a high resolution is required to test for genetic differences. 


\subsection{Genetic analyses below the species level to assess the genetic integrity of cryopreserved microalgae}

Traditionally, microalgal cultures are maintained in an actively metabolizing state referred to as subculturing or serial transfer (Day and Brand 2005, Lorenz et al. 2005, Fig. 1.2a). Cryopreservation is an effective alternative because after the initial cryopreservation, no regular handling procedures are involved, minimizing the introduction of contaminations or the possibility to mix up cultures (Day and Brand 2005). Cryopreservation has come to be the method of choice for the long term conservation of microalgae because theoretically it should guarantee genetically stable cultures over decades (Benson 2004, Brand and Diller 2004). However, one could also argue that the various steps of the cryopreservation procedure (Fig. 1.2b and c) cause considerable stress to algal cells, e.g. toxicity of the cryoprotectant, osmotic shock, and intracellular ice formation (Day et al. 2000) that may lead to genomic alterations. Very sensitive molecular markers are needed to assess the genetic integrity of the same strain before and after cryopreservation. The SAG culture collection was partner in the European research project COBRA (the COnservation of a vital European scientific and Biotechnological Resource: microAlgae and cyanobacteria, QLRI-CT-2001-01645, November 2001 - March 2005). In the course of this project, cryopreservation facilities were established at the SAG culture collection and up to now more than 400 strains of microalgae and cyanobacteria have been successfully cryopreserved. An important goal of the present study was to check for the genetic integrity in selected cryopreserved culture strains.

\subsection{Amplified fragment length polymorphism}

\subsubsection{Overview of the technique}

AFLP permits the simultaneous analysis of many loci widely spread over the entire genome, without prior sequence knowledge of the organisms under study. The AFLP technique involves the restriction of genomic DNA with endonucleases, followed by ligation of adaptors (double-stranded oligonucleotides) complimentary to the restriction sites and selective PCR amplification of a subset of the adapted restriction fragments. The amplified fragments are separated by electrophoresis. Visualisation of fragments depends on the employed electrophoresis system. In the present study a capillary sequencer was used that works with fluorescence-labelled primers, and the AFLP banding patterns were displayed in electropherograms (Fig. 1.1). 


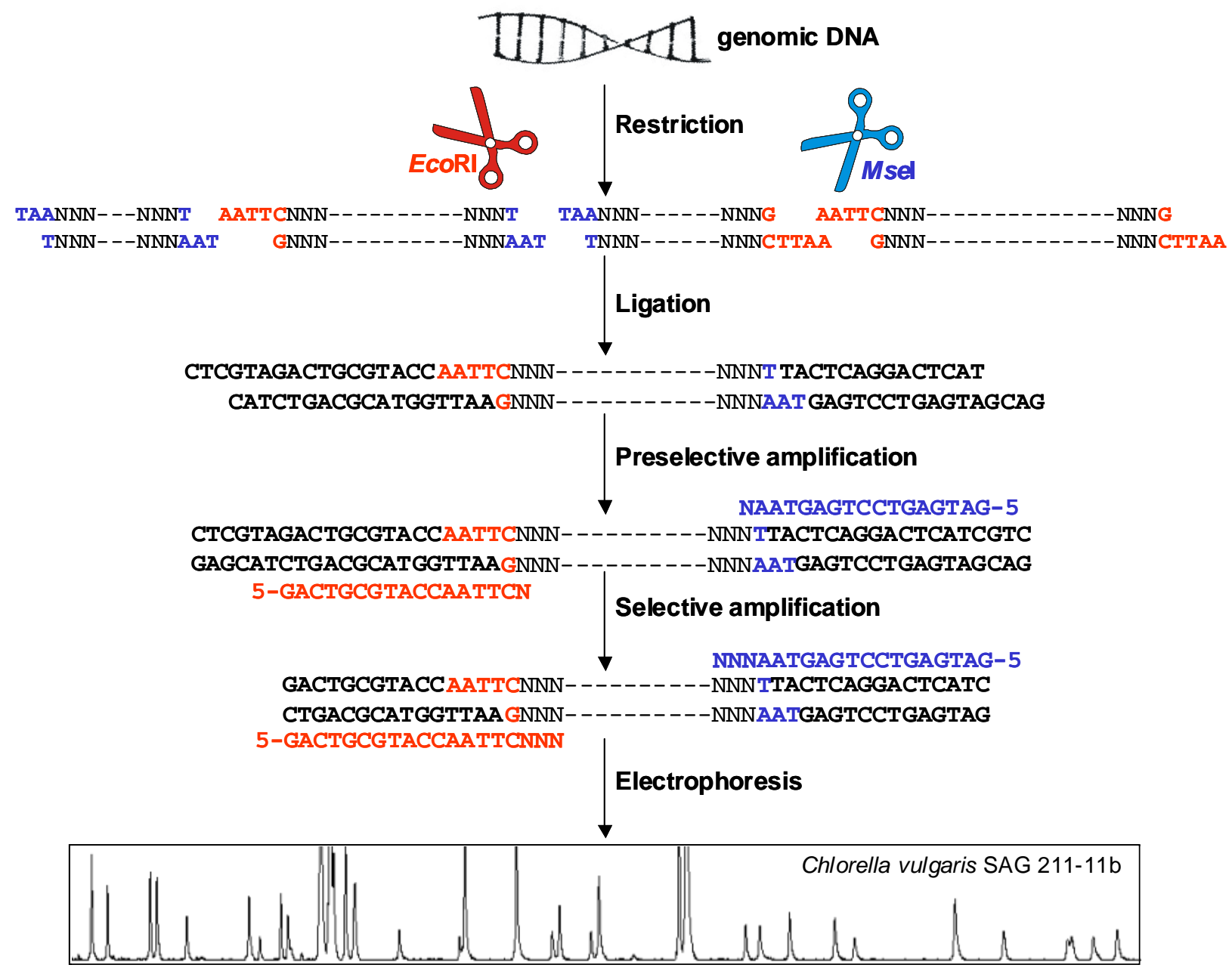

Fig. 1.1 Overview of the AFLP technique. Restriction: Genomic DNA is digested with two restriction enzymes, one "frequent cutter" and one "rare cutter" enzyme. The "frequent cutter" enzyme has a recognition sequence of four base pairs (e.g. Msel T'TAA, in blue) and the "rare cutter" of six base pairs (e.g. EcoRI, G'AATTC, in red). Ligation: Double-stranded adapters (in black) are ligated to the ends of the generated DNA fragments to create target sites for primer annealing in the following PCR step. Two different kinds of adapters are ligated, one with a complementary end to the fragments generated by the "rare cutter" and the other with a complementary end to the fragments generated by the "frequent cutter" enzyme. Preselective amplification: A subset of the restriction fragments is specifically amplified by using a selective nucleotide $(\mathrm{N})$ at the 3' ends of the AFLP primers. Two AFLP primers are used, one for the EcoRI- and one for the Msel-adapters. A four-fold reduction of amplified fragments is achieved with each selective nucleotide. Selective amplification: The PCR products of the preselective amplification are used as template for a second PCR using primers both having up to three selective nucleotides (NNN), to allow to adjust the amount of fragments that are amplified. This depends on the genome size of the organism under study. Electrophoresis: Fragments obtained after selective amplification are separated on a polyacrylamide gel or on a capillary sequencer and visualised using radioactively or fluorescently labelled primers, or are silver stained. An electropherogram for the strain Chlorella vulgaris SAG 211-11b produced by a capillary sequencer is shown here. 

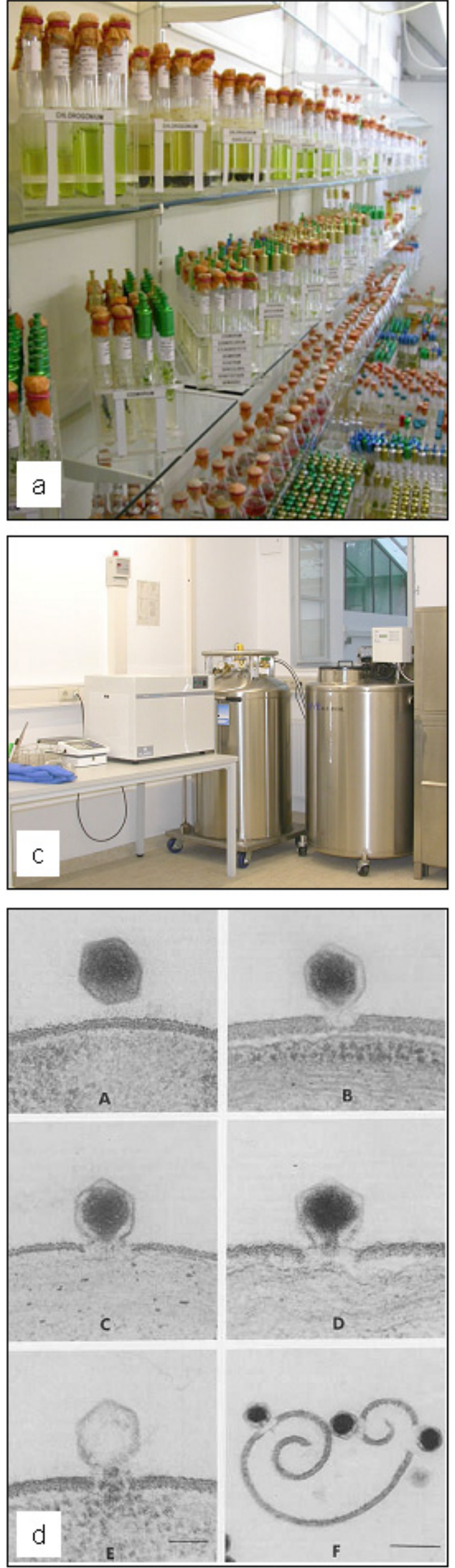

\section{1.) pre-cryo cultures:}

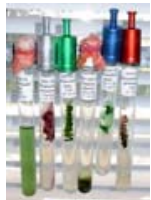

2.) +cryoprotectant in culture medium:

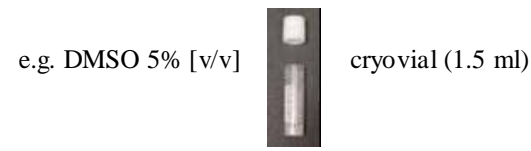

3.) two-step controlled cooling:

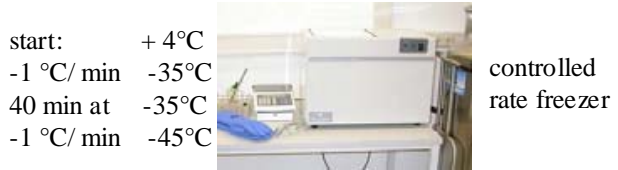

4.) storage in vapour phase of liquid nitrogen:

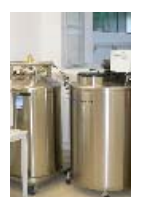

storage vessel

5.) thawing, water bath $45^{\circ} \mathrm{C} 3 \mathrm{~min}$

6.) dilution with fresh medium and recovery for $12 \mathrm{~h}$ in darkness

7.) normal culture conditions 2-3 weeks

8.) regrown post-cryo cultures:

b

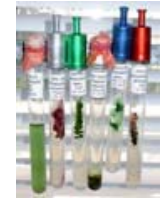

Fig. 1.2 Culture facilities for subculturing, overview of cryopreservation procedure and viral infection. (a) Subculture room at SAG culture collection. (b) Overview of cryopreservation protocol used in the present thesis (cf. Chapter 5). (c) Cryopreservation facilities at SAG culture collection. (d) Infection of Chlorella strain NC64A by PBCV-1 (cf. Chapter 6). (A) Viral particle in close proximity to the alga. ( $B$ and C) Attachment of PBCV-1 to the algal wall and digestion of the wall at the point of attachment. (D) Viral DNA beginning to enter the host. (E) An empty viral capsid remaining on the surface of the host. (F) PBCV-1 attachment and dissolution of a Chlorella cell wall fragment.

(d) = (c) 1999-2000 James L. Van Etten,

University of Nebraska, Lincoln,

http://plantpath.unl.edu/facilities/Virology/index.html 

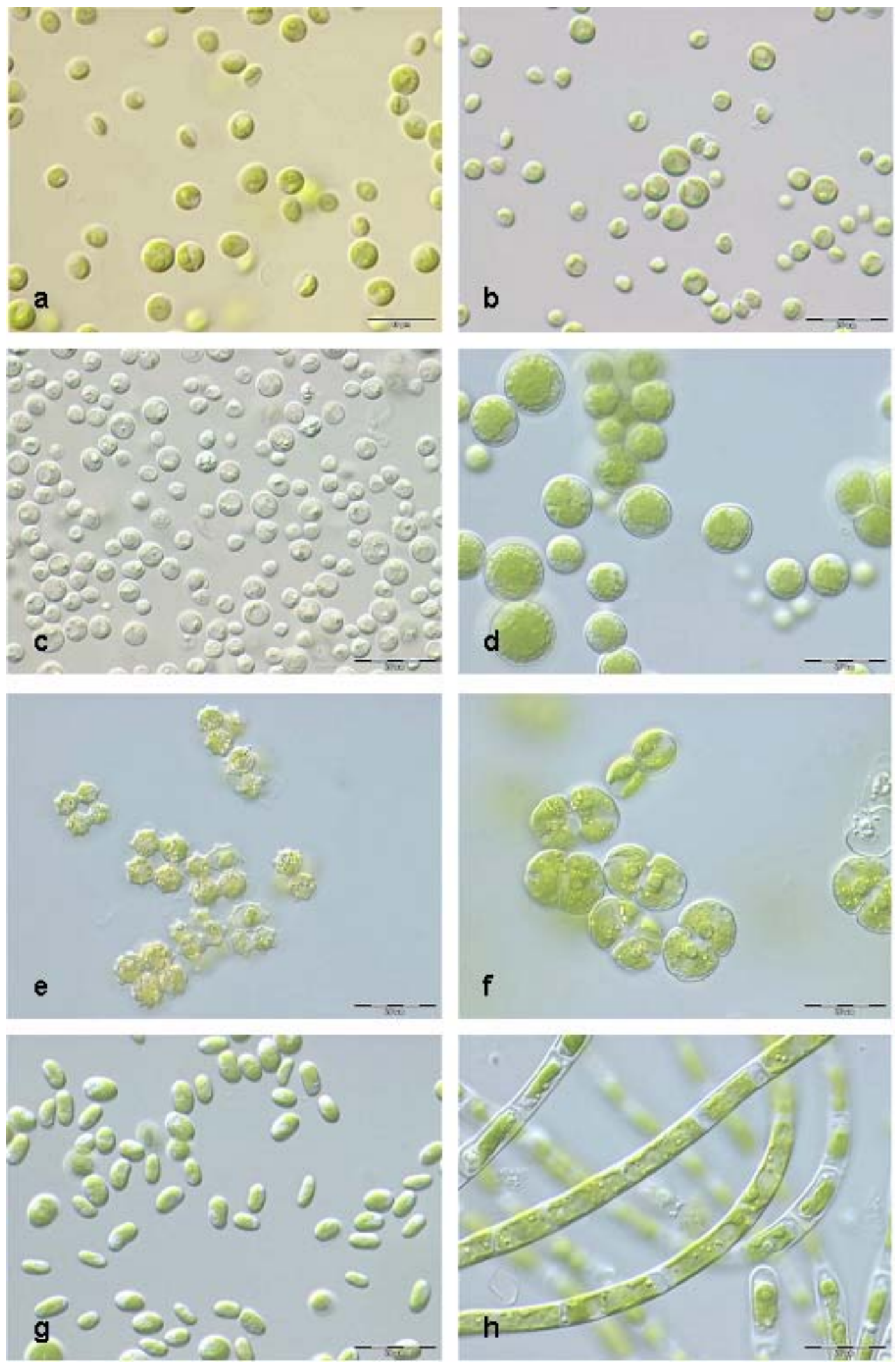

Fig. 1.3 Examples of microalgal strains investigated in the this study. (a) Chlorella vulgaris SAG 211-11b (Chapters 2 and 5). (b) Parachlorella kessleri SAG 211-11h, wildtype strain (Chapters 3 and 5). (c) Parachlorella kessleri SAG 211-11h/20, radioisotope-induced mutant (Chapters 3 and 5). (d) Trebouxia asymmetrica SAG 48.88 (Chapter 5). (e) Coelastrum morum Hg 1999-5 (Chapter 5). (f) Cosmarium cucumis SAG 612-10 (Chapter 5). (g) Coccomyxa arvernensis SAG 216-1 (Chapter 5). (h) Uronema belkae SAG 34.86 (Chapter 5). Scale bar: 20 $\mu \mathrm{m}$. 


\subsection{Aims of thesis}

The aim of the present thesis was to analyse microalgal culture strains below the species level. This was important to improve the abilities of service culture collections of algae to preserve biodiversity more efficiently and to provide their user community with correctly identified and clean organisms. As examples commonly used microalgal strains of great value in applied research were investigated with AFLP.

Multiple and duplicate strains of Chlorella vulgaris (Fig. 1.3a) were investigated with AFLP. For this first test example, the AFLP protocol had to be improved and a standard protocol was developed including three replications of AFLP patterns per strain. In addition, manual and automatic evaluation of banding patterns were compared (Chapter 2).

Discrimination at an even further level of resolution, namely between mutants of the same isolate, was investigated with AFLP for pigment mutants of Parachlorella kessleri (Fig. 1.3b and c). Genetic differences were found among mutants (induced by UV light, radioisotope treatment or $\mathrm{x}$-ray irradiation) that showed clear phenotypic differences in pigment composition and carotenoid biosynthesis (Chapter 3).

A genetic characterization of a wildtype strain and mutants of Dunaliella salina that were contaminated with bacteria and/or fungi was performed with AFLP. To achieve this, banding patterns generated from cultures with differing amounts of algae/contaminants were compared (Chapter 4).

The genetic integrity of cyropreserved microalgae was assessed with AFLP. Banding patterns of strains prior to and after the cryopreservation process as well as after several rounds of freezing/thawing were compared for algae that differed in their sensitivity towards the cryopreservation procedure. Prior to this, the reproducibility of AFLP was tested for an extended sample of microalgae of different morphology and taxonomy. Examples of strains are shown in Fig. 1.3 (Chapter 5).

The capability of AFLP to detect viral contaminations in microalgae was tested. Viral infections are difficult to detect in microalgal culture strains, but have been reported for a high number of taxa. AFLP banding patterns of a pure host algal culture, a pure culture of its virus and the infected algal culture were compared (Chapter 6, for lytic infection see Fig. 1.2d). 


\section{Distinction of isolates among multiple strains of Chlorella vulgaris and testing conspecificity with amplified fragment length polymorphism and ITS rDNA sequences*}

\subsection{Abstract}

Cultures of Chlorella vulgaris Beijerinck are of great value for applied research. Multiple strains per species are available from public culture collections, often with the same isolate being maintained in parallel at different collections under different culture regimes. To unravel genomic variation and to identify unique genotypes among such multiple strains two approaches were employed on a sample of 29 strains from five culture collections. Except for two strains, ITS rDNA sequence data substantiated conspecificity of the studied strains and only minor sequence differences were found with the authentic 'Beijerinck isolate'. Amplified fragment length polymorphism (AFLP) detected considerable genomic variation when rDNA sequences were invariant. Band detection and the construction of a binary matrix from AFLP patterns for phylogenetic analyses were fully automated, but comparison of similar patterns still required manual refinement. AFLPs distinguished eleven unique genotypes and provided robust support for the presence of five cryptic species. This finding advocates the need to carefully record which strain has been used in any experiment or applied research. The genomic variation may also correspond to differences in physiological/biochemical properties. No genomic differences could be detected among duplicate strains of the same isolate that were maintained by continuous subculturing over many decades, or stored at ultra-low temperatures.

\subsection{Introduction}

In public culture collections the same species of microscopic alga is often represented by multiple strains. This constitutes a serious concern for the culture collections, because it could mean that a considerable proportion of their holdings of living algae may be identical replicates. For example, 16\% (360 strains) of the total holdings of the SAG culture collection correspond to just 49 species of which each is represented by five or more strains (calculation taking into account mating pairs of species with sexual reproduction). Yet, the amount of 'redundant' genotypes (i.e. identical replicates) among these multiple strains is not known. In addition, strains of a certain species at one culture collection may also be present as duplicates in another, or even several other collections. For instance, from the extensive number of algal

*This Chapter has received a positive review (Müller et al. 2005, Journal of Phycology). 
species that E.G. Pringsheim, one of the early pioneers in algal culturing (Preisig and Andersen 2005), isolated, the SAG culture collection maintains 152 strains that are also kept at up to four other collections (Day et al. 2004). To improve the value of algal culture collections to the user community an important consideration is to assure conspecificity of the multiple strains per species and then to identify unique genotypes among them. The latter is most important for culture collections when accessioning a new strain in order to avoid excessive replication in their holdings.

Chlorella vulgaris Beijerinck (Fig. 1.3a) appears typical of an algal species for which quite a number of multiple and duplicate strains is available, and it is investigated here as an example. Obviously, C. vulgaris is of a great biotechnological potential for producing valuable substances for the feed, food, cosmetics and neutraceutical/pharmaceutical industries. It has been found particularly suitable for large-scale microalgal biomass production (Pulz 2001) and applications including: tests metal ion toxicity (e.g. Scragg and Bonnett 2002), waste water treatment (Tam et al. 1994, Mallick 2002, 2003, De-Bashan et al. 2004), the production of a new PVC-Chlorella composite material (Zhang et al. 2000) or as an additive in sustainable fuel (Scragg et al. 2003). Strains 211-11b and 211-12 from the SAG as well as their duplicates from the CCAP and UTEX culture collections are used in standard ecotoxicological algal growth inhibition toxicity tests (OECD 1984, Day et al. 1999). The first synchronous culture of a microalga was developed with C. vulgaris strain C-27 from IAM culture collection (Tamiya et al. 1953) and the complete chloroplast genome has been sequenced for the same strain (Wakasugi et al. 1997).

C. vulgaris was first described and isolated into pure (axenic) culture by the Dutch microbiologist M.W. Beijerinck in 1889 (Beijernick 1890). This particular isolate, which is still available from at least three major culture collections today (Table 2.1), forms an important reference (authentic material) for the definition of the species. At present, a total of 55 strains of $C$. vulgaris is available from six major public culture collections (ATCC, CCALA, CCAP, NIES, SAG and UTEX) and this ensures ready world-wide availability of $C$. vulgaris as a genetic resource. Most of these multiple strains are 'duplicates', i.e. clones of the same origin which have been maintained in parallel at separated culture collections over several decades. At the SAG culture collection 15 strains of $C$. vulgaris are available, of which 10 are also maintained as duplicates in other culture collections (Table 2.1). There are several reasons why genomic diversity can be expected among the multiple strains of $C$. vulgaris as many of them represent isolates of different origins, i.e. they were isolated from geographically distant localities and different habitats. Unfortunately, it is often impossible to 
trace their origins as many strains that have been held for decades often have poor data records - a situation that is paralleled by many other microalgal strains available from culture collections. Additional diversity could have been introduced 'artificially', i.e. by different maintenance methods and culture regimes at different collections. Algal strains are undoubtedly affected by selective pressures and/or genetic drift caused by continuous subculturing over thousands of generations under different culture regimes (Brand and Diller 2004, Day and Brand 2005), but this has not been examined yet. Also, over many decades human error may result in a higher likelihood of mislabeling or a "mix up" on performing a serial transfer (Lorenz et al. 2005). Furthermore, the same clone may have been stored at an ultra-low temperature (cryopreservation, Karlsson and Toner 1996) in one culture collection whilst it was also maintained in actively metabolizing state in another collection. This may lead duplicates of the same clone to at two culture collections to be genetically different. Because most $C$. vulgaris strains have been maintained for many decades they are ideal for studying such putative effects which may increase over time.

Sensitive molecular methods are required to unravel genomic variation within strains of a single species of microscopic alga. ITS rDNA sequence analyses are commonly used for phylogenetic inference at the generic and intrageneric levels (Álvarez and Wendel 2003) and have been applied to a broad range of algae (Coleman and Mai 1997, Kooistra et al. 2002, Behnke et al. 2004). Amplified fragment length polymorphism (AFLP, Vos et al. 1995) has proved to be well suited for provision of resolution where rDNA sequence data were not sensitive enough. The method has been increasingly employed for algae, i.e. to resolve genetic distances among geographic isolates and to delineate species of marine macroalgae (Schaeffer et al. 2002, Murphy and Schaffelke 2003, Erting et al. 2004), for marine microalgae (De Bruin et al. 2004, John et al. 2004) and charophytes (Mannschreck et al. 2002), but to our knowledge it has been applied to freshwater microalgae only once so far (Werner et al. 2001). AFLP has also successfully been used to evaluate genetic diversity and redundancy in a germplasm collection of cassava (Manihot esculenta Crantz) where AFLP markers were found very effective at selecting unique genotypes as well as for the identification of genomic repetitiveness (Chavarriaga-Aguirre et al. 1999).

To investigate the genomic variation among multiple strains of $C$. vulgaris in this study, AFLPs and/or ITS rDNA sequences were determined for 29 strains from five culture collections. For the fifteen strains studied from the SAG culture collection previous measurements of their GC-contents (Kessler 1976), DNA hybridization (Huss et al. 1989) and highly similar cell wall compositions (Takeda 1988, 1991, 1993) have already indicated their 
close relationship among each other. In addition, all of their duplicate strains from the CCAP, three from the CCALA and two from the UTEX culture collections were included in this study as well as strain CBS 15-2075 which has been used more frequently in biotechnological applications (e.g. Tam et al. 1998, Lam et al. 1999, Wong et al. 2000). Apart from two exceptions, conspecificity of the strains with $C$. vulgaris was substantiated by the rDNA sequence data, but considerable genomic variation was found with AFLPs where sequences of the ITS regions gave no further resolution.

\subsection{Materials and Methods}

\subsubsection{Investigated strains}

All 15 Chlorella vulgaris strains available from the SAG (www.epsag.unigoettingen.de) and strain 15-2075 from Carolina Biological Supply, Burlington, USA (CBS) had their ITS-1,2 rDNAs sequenced (Table 2.1) to check for any sequence differences with the authentic 'Beijerinck' isolate, i.e. strain SAG 211-11b. The corresponding duplicate strains from the CCAP (www.ccap.ac.uk), except for the CCAP strains 211/11P, 211/11Q, 211/11S, and 211/19, and three from CCALA (www.butbn.cas.cz/ccala/ccala.htm) were sequenced over their ITS regions (Table 2.1). To check for genetic differences at an even higher resolution, the 15 SAG strains of $C$. vulgaris, all duplicate strains from CCAP, as well as two additional corresponding duplicate strains from the UTEX (www.utex.org) (Table 2.1) were used for the AFLP studies. All cultures were checked for axenicity on media containing proteose peptone and saccharose (agarized Trebouxia Organic Medium, Ahmadjian 1967) and were maintained on the same medium under standard culture conditions (12/12h light/dark regime at $18^{\circ} \mathrm{C}$ ) prior to DNA extraction. For CBS 15-2075 and the three tested strains from CCALA contaminations by yeasts and/or bacteria were found, which excluded these strains from the AFLP studies.

\subsubsection{DNA extraction and rDNA sequencing}

Algal cells were mechanically disrupted by shaking in the presence of glass beads (Friedl 1995), or were frozen in liquid nitrogen and ground to powder. Genomic DNA was extracted using the "Invisorb Spin Plant Mini Kit (Invitek, Berlin, Germany) following the instructions given by the manufacturer. For the AFLP studies genomic DNA was extracted twice from each strain on two different days in order to detect variations in AFLP banding patterns due to differences in the extraction processes. For the AFLP fingerprints, DNA needs 
to be of optimal quality to allow complete digestion and this step is crucial for the production of high quality AFLP fingerprints. Only samples with high molecular DNA, i.e. those migrating at the same level with the $20 \mathrm{~kb}$-fragment of lambda DNA standard in agarose gel electrophoresis, were processed further. Amplification of ITS rDNA sequences was carried out as described in (Friedl 1996), and the Dye Terminator Cycle Sequencing Kit v2.0 (Applied Biosystems, Foster City, CA, USA) was used to separate the sequencing reactions on a ABI Prism 3100 (Applied Biosystems) automated sequencer. The Genbank accessions for the ITS rDNA sequences determined in this study are given in Table 2.1. These sequences were manually aligned using the program BioEdit v.6.0.7 (Hall 1999). To compare the ITS rDNA sequences for the deviating strains CCAP 211/11F and CBS 15-2075 (accession numbers AY591514 and AY948419) with available sequences BLASTn searches (Altschul et al. 1997) at the National Center for Biotechnology Information (NCBI, http://www.ncbi.nlm.nih.gov/) were performed. To assess the identity of strain CCAP 211/11F its 18S rDNA was amplified Friedl and O'Kelly (2002) and sequenced using standard sequencing primers (accession number AY591515) and subjected to BLASTn.

\subsubsection{AFLP reactions}

Restriction and ligation were carried out simultaneously in a single reaction (Mannschreck et al. 2002). Approximately 50-400 ng (5.5 $\mu \mathrm{L}$ ) of high molecular DNA were incubated with $5 \mathrm{U}$ EcoRI, $1 \mathrm{U}$ MseI and 1 Weiss Unit T4 DNA ligase (all New England Biolabs, Frankfurt/M., Germany) in T4 Ligase buffer with $55 \mathrm{mM} \mathrm{NaCl}, 0.55 \mu \mathrm{g}$ bovine serum albumin, EcoRI- and MseI-adapters (5 and 50 pmol, adapter sequence as in Vos et al. 1995) in a total volume of $11.0 \mu \mathrm{L}$ for 3 hours at $37^{\circ} \mathrm{C}$. The completeness of the digestion was checked on an agarose gel. Reactions were diluted five-fold with distilled water and then $4 \mu \mathrm{L}$ were used for the pre-selective amplification with primers MseI+0 and EcoRI+0 (2.5 pmol each, primer sequence as in Vos et al. 1995), Taq Polymerase (0.5 U, Silverstar, Eurogentec, Seraing, Belgium), the appropriate $10 \mathrm{x}$ reaction buffer (Eurogentec, Seraing, Belgium) with $1.5 \mathrm{mM} \mathrm{MgCl}_{2}$ and dNTPs (0.2 mM each, Hybaid, Heidelberg, Germany) in a total volume of $20 \mu \mathrm{L}$. The amplification profile was $3 \mathrm{~min}$ at $94^{\circ} \mathrm{C}$, followed by 20 cycles of $20 \mathrm{sec}$ at $94^{\circ} \mathrm{C}$, $30 \mathrm{sec}$ at $56^{\circ} \mathrm{C}$ and $120 \mathrm{sec}$ at $72^{\circ} \mathrm{C}$. Quality of the pre-selective amplification was checked on an agarose gel and were then diluted twenty-fold with water prior to the selective amplification step. Dilution was decreased down to five-fold if concentration of the amplified DNA was lower than in other samples. In this second amplification step $4 \mu \mathrm{L}$ of the diluted pre-selective amplification reaction were used in total reaction volume of $20 \mu \mathrm{L}$ as a template 
for three primer combinations, i.e. primers EcoRI+A (7 pmol), EcoRI+C (5 pmol) and EcoRI+G (5 pmol) were combined with $M s e \mathrm{I}+\mathrm{C}$, MseI+G or MseI+0 (all $10 \mathrm{pmol}$ ) primer. The different EcoRI-primers were either labeled with the 6-FAM, VIC, or NED fluorochromes (Applied Biosystems). For the selective amplification conditions were identical to those in the preselective amplification step. The profile was $3 \mathrm{~min}$ at $94^{\circ} \mathrm{C}, 10$ cycles of $20 \mathrm{sec}$ at $94^{\circ} \mathrm{C}, 30 \mathrm{sec}$ at $65^{\circ} \mathrm{C}$ with decreasing for $1^{\circ} \mathrm{C}$ after each cycle down to $56^{\circ} \mathrm{C}$ and $120 \mathrm{sec}$ at $72^{\circ} \mathrm{C}$, followed by 20 cycles of $20 \mathrm{sec}$ at $94^{\circ} \mathrm{C}, 30 \mathrm{sec}$ at $56^{\circ} \mathrm{C}$ and 120 sec at $72^{\circ} \mathrm{C}$. To check the reproducibility of the resulting banding patterns, the complete AFLP reaction was done twice with the first DNA extraction and third time with the second DNA extraction. The three replicate reactions were done for each strain and on different days.

\subsubsection{AFLP fragments sizing and evaluation}

The fluorescently labeled AFLP fragments were separated by capillary electrophoresis using an ABI Prism 3100 automatic sequencer (Applied Biosystems). Sizing of fragments with lengths of 35 to 500 base pairs was carried out using the GeneScan-500 [LIZ] standard (Applied Biosystems) and the program GeneScan (Applied Biosystems). An automated evaluation using the program GenScanner (D. Hepperle, unpubl.) was performed for the detection of fragments. This program was also used to construct a binary matrix for the subsequent reconstruction of phylogenies from the presence/absence of fragments. GenScanner uses for the automated evaluation a consensus of the banding patterns from three independent AFLP reactions per strain that was built by scoring only fragments present in at least two of the three replicates above a threshold of 50 relative fluorescent units (rfu). We also tested to adjust the threshold at different percent levels relative to the average fluorescence intensity of all fragments in a sample and to the peak height of the strongest fragment. The construction of binary matrices in GenScanner is based on optimal bin locations which are determined by identifying local maxima. The latter are detected from a spreadsheet divided into $0.1 \mathrm{bp}$ categories and where each fragment occupies the cell of its size plus/minus five 0.1 bp categories. Bins are constructed in 1.0 bp distances to each other and are defined to contain the highest possible number of fragment entries. For each determined bin its size value is calculated as a mean value over all fragments contributing to this bin. This procedure is carried out for parallel samples of the same strain ('bins') as well as for comparison of bins from different strains ('hyperbins'). The resulting binary matrix is exported in NEXUS format. Those strains that were grouped into a clade in phylogenetic analyses and, therefore, exhibited rather similar banding patterns, were further compared by 
eye. The three fragment patterns obtained for each strain were compared and only those fragments that could be detected as present in at least two replicates were counted; no threshold value of relative fluorescent units was used. For both evaluation methods fragments with sizes of 100 to 500 bp were analyzed resulting in an average of 150 fragments per sample for automated and 164 fragments per sample for visual comparison.

\subsubsection{Phylogenetic analyses of AFLP data}

The binary matrix obtained from the GenScanner program contained 490 columns that corresponded to 472 variable and 424 parsimony-informative sites. Analyses were carried out using PAUP* V4.0b10 (Swofford 2001). A pairwise distance matrix was constructed using the restriction-site distance of Nei \& Li (1979) and this was used in a neighbor-joining (NJ) analysis combined with a bootstrap analysis with 2000 bootstrap pseudo-replicates. For maximum parsimony (MP) analyses, the binary matrix was equally weighted and robustness of the tree was also inferred by bootstrap analysis including 2000 replicates. For MP the heuristic search method was used with starting trees built stepwise with 10 random additional replicates, using the tree bisection-reconnection branch-swapping algorithm to find the best tree. The best-scoring trees were held at each step. 


\section{$2.4 \quad$ Results}

\subsubsection{ITS rDNA analyses}

The ITS-1,2 rDNA sequences were determined for the fifteen strains from SAG, seven duplicate strains from CCAP and CCALA and strain CBS 15-2075 in order to test for conspecificity, i.e. close relationship to the authentic 'Beijerinck isolate' (Table 2.1). Except for two strains, CCAP 211/11F and CBS 15-207, the sequences were very similar to the 'Beijerinck isolate'. There were no more then ten sequence positions variable among the studied strains (Fig. 2.1). Lengths of their ITS1-5.8S-ITS2 regions were identical (291-159245 nucleotides) except for one position difference in ITS-1.

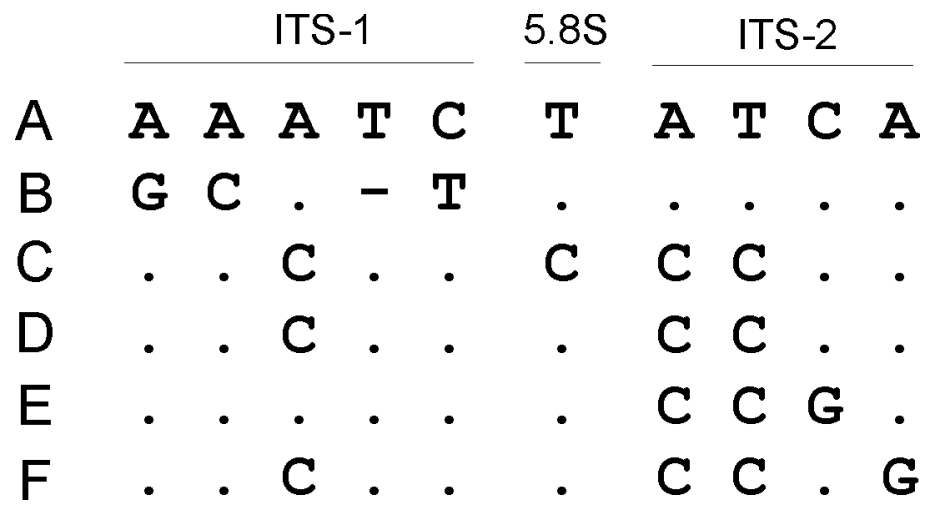

Fig. 2.1 Variable positions of ITS1-5.8S-ITS2 sequences from multiple strains of $C$. vulgaris that define six different ITS-variants (A-F). Differences at a certain sequence position with variant $A$ (the 'Beijerinck isolate', see text) are shown by a nucleotide, sequence identity by a dot, and a gap marks lack of a nucleotide.

The sequence differences grouped the strains into six ITS variants, A-F (Fig. 2.1). The majority of strains fell into two ITS variants, C and D, with only one sequence position difference between them. Closest to the 'Beijerinck isolate' were ITS variants D and E with just three sequence positions difference, whilst variant B was most distant to it with six sequence differences. No differences were found between the ITS sequences of the three duplicate strains representing the 'Beijerinck isolate', SAG 211-11b, CCAP 211/11B and CCALA 269. Also, each of the four other sequenced pairs of duplicate strains (SAG 2.80 and CCALA 268, SAG 211-1e and CCALA 262, SAG 211-12 and CCAP 211/12, SAG 211-11j and CCAP 211/11J) had no differences in their ITS regions. The ITS1-5.8S-ITS2 region of strain CCAP 211/11F was shorter than in the other C. vulgaris strains, i.e. 262-159-235 nucleotides. 
For the ITS-2 sequence of CCAP 211/11F the highest level of similarity was revealed with the corresponding sequence from C. sorokiniana AY323463 (strain CCALA Prag A14) in BLASTn searches, but the alignment was ambiguous because of considerable sequence differences. To further assess the phylogenetic position of strain CCAP 211/11F, its 18S rDNA sequence was determined. Phylogenetic analyses placed this strain within the "Chlorella-clade" of the Chlorellaceae sensu Krienitz et al. (2004), but its exact position within that clade was ambiguous (analyses not shown). Also in strain CBS 15-2075 the ITS15.8S-ITS2 region was shorter than in C. vulgaris, i.e. 243-159-246 nucleotides. The ITS-2 sequence of strain CBS 15-2075 was fully identical with the corresponding sequence from Parachlorella kessleri AY323477 (strain SAG 211-11g) which is phylogenetically more distant to C. vulgaris than C. sorokiniana (Krienitz et al. 2004).

\subsubsection{AFLP analyses}

The AFLP technique was employed to obtain a resolution enhanced over rDNA sequencing. The AFLP analyses included all C. vulgaris strains that were sequenced over their ITS-1,2 regions except for strains from CCALA and CBS 15-2075 which were not axenic, and additional duplicate strains from CCAP and UTEX (Table 2.1). From the nine tested primer combinations, four yielded satisfactory AFLP patterns with a reasonable number of fragments, i.e. EcoRI+A/MseI+C, EcoRI+A/MseI+G, EcoRI+C/MseI+C, and EcoRI+C/MseI+G. Multiplex PCR reactions where three EcoRI-primers were combined with one MseI primer in the same reaction showed the same results as when performed in separate reactions for each primer combination. By visual comparisons of the AFLP patterns five groups could be clearly distinguished (Fig. 2.2). Patterns within a group appeared more similar than patterns between the groups. In automated fragment evaluation no strain was identical with another one. This method assigned every single strain to a separate lineage in the phylogenetic analyses and the 24 strains of $C$. vulgaris were separated into five clades (Fig. 2.3A). The grouping by AFLP was largely congruent with the grouping by ITS variants, i.e. each clade in the AFLP-based phylogenies was defined by a certain ITS variant with two exceptions, strains SAG 9.88 and SAG 2.80 (Fig. 2.3C). Monophyly of each of the five clades was well supported with $100 \%$ in all bootstrap replicates. The relationships between the clades were well resolved, i.e. the sister-group relationship of clades 3 and 4 and their shared origin with clade 5 were well supported in bootstrap tests. However, the relative positions of clades 1 and 2 were ambiguous. The majority of the strains belonged to two clades, 3 and 4, and relationships within both clades were mostly unresolved (Fig. 2.3A). 
Table 2.1 The strains and isolates of Chlorella vulgaris used in ITS rDNA sequencing and AFLP analyses, available information about their origins, GenBank acccesion numbers, ITS variants and AFLP clades. For culture collection acronyms see text and list of abbreviations.

\begin{tabular}{|c|c|c|c|c|c|c|}
\hline Strain & Locality & Isolator & $\begin{array}{c}\text { Year } \\
\text { of isolation }\end{array}$ & $\begin{array}{c}\text { GenBank } \\
\text { accession } \\
\text { ITS } \\
\end{array}$ & $\begin{array}{c}\text { ITS } \\
\text { variant }\end{array}$ & $\begin{array}{l}\text { AFLP } \\
\text { clade }\end{array}$ \\
\hline SAG 211-11b & \multirow{3}{*}{$\begin{array}{l}\text { Delft, Netherlands, } \\
\text { authentic strain }\end{array}$} & \multirow{3}{*}{$\begin{array}{l}\text { M. W. } \\
\text { Beijernick }\end{array}$} & \multirow{3}{*}{1889} & AY591508 & A & 1 \\
\hline CCAP 211/11B & & & & AY591507 & A & 1 \\
\hline CCALA 269 & & & & AY591506 & A & n.d. \\
\hline SAG 211-11f & \multirow{2}{*}{ Dorking, UK } & \multirow{2}{*}{$\begin{array}{c}\text { E. G. } \\
\text { Pringsheim }\end{array}$} & \multirow{2}{*}{1939} & AY591511 & B & 2 \\
\hline CCAP 211/11F & & & & AY591514 & n.a. & n.a. \\
\hline SAG 211-1e & \multirow{2}{*}{$\begin{array}{c}\text { unknown, received } \\
\text { from H. Tamiya }\end{array}$} & \multirow{2}{*}{ H. Gaffron } & \multirow{2}{*}{1959} & AY591512 & B & 2 \\
\hline CCALA 262 & & & & AY591513 & B & n.d. \\
\hline SAG 30.80 & $\begin{array}{l}\text { unknown, received } \\
\text { from H. Senger as } \\
\text { "Tamiya-Strain" }\end{array}$ & unknown & $\begin{array}{c}\text { before } \\
1965\end{array}$ & AY591510 & B & 2 \\
\hline SAG 211-11j & \multirow{3}{*}{ unknown } & \multirow{3}{*}{ W. Rohde } & \multirow{3}{*}{ unknown } & AY591498 & $\mathrm{C}$ & 3 \\
\hline CCAP 211/11J & & & & AY591497 & $\mathrm{C}$ & 3 \\
\hline UTEX 265 & & & & n.d. & n.d. & 3 \\
\hline SAG 211-19 & \multirow{2}{*}{$\begin{array}{l}\text { Göttingen, } \\
\text { Germany }\end{array}$} & \multirow{2}{*}{ H. v. Witsch } & \multirow{2}{*}{1946} & AY591499 & C & 3 \\
\hline CCAP 211/19 & & & & n.d. & n.d. & 3 \\
\hline SAG 211-11t & $\begin{array}{l}\text { Werden, Germany, } \\
\text { received from } \\
\text { G.Ruschmann }\end{array}$ & H. Weiß & $\begin{array}{c}\text { before } \\
1956\end{array}$ & AY591493 & C & 3 \\
\hline SAG 211-8m & $\begin{array}{l}\text { unkown, received } \\
\text { from G. } \\
\text { Ruschmann } \\
\end{array}$ & O. Warburg & unknown & AY591494 & C & 3 \\
\hline SAG 211-11q & \multirow{2}{*}{ unknown } & \multirow{2}{*}{$\begin{array}{l}\text { V. Czurda } \\
\text { (?) }\end{array}$} & \multirow{2}{*}{$\begin{array}{l}\text { before } \\
1946\end{array}$} & AY591504 & $\mathrm{D}$ & 4 \\
\hline CCAP 211/11Q & & & & n.d. & n.d. & 4 \\
\hline SAG 211-11s & \multirow{2}{*}{$\begin{array}{l}\text { unknown, received } \\
\text { from A. Pirson }\end{array}$} & \multirow{2}{*}{ unknown } & \multirow{2}{*}{$\begin{array}{c}\text { before } \\
1952\end{array}$} & AY591509 & $\mathrm{D}$ & 4 \\
\hline CCAP 211/11S & & & & n.d. & n.d. & 4 \\
\hline SAG 211-12 & \multirow{3}{*}{$\begin{array}{l}\text { unknown, authentic } \\
\text { strain of } C \text {. } \\
\text { vulgaris var. viridis }\end{array}$} & \multirow{3}{*}{ R. Chodat } & \multirow{3}{*}{ unknown } & AY591503 & $\mathrm{D}$ & 4 \\
\hline CCAP 211/12 & & & & AY591502 & $\mathrm{D}$ & 4 \\
\hline UTEX 30 & & & & n.d. & n.d. & n.d. \\
\hline SAG 211-81 & unknown & R. Emerson & unknown & AY591501 & $\mathrm{D}$ & 4 \\
\hline SAG 2.80 & \multirow{2}{*}{ unknown } & \multirow{2}{*}{ H. Gaffron } & \multirow{2}{*}{ unknown } & AY591495 & $\mathrm{D}$ & 3 \\
\hline CCALA 268 & & & & AY591496 & $\mathrm{D}$ & n.d. \\
\hline SAG 9.88 & Madrid, Spain & I. Orus & $\begin{array}{c}\text { before } \\
1986\end{array}$ & AY591500 & E & 4 \\
\hline SAG 211-11p & \multirow{2}{*}{ Lund, Sweden } & \multirow{2}{*}{ S. Algeus } & \multirow{2}{*}{1942} & AY591505 & $\mathrm{F}$ & 5 \\
\hline CCAP 211/11P & & & & n.d. & n.d. & 5 \\
\hline CBS 15-2075 & $\begin{array}{c}\text { unknown, received } \\
\text { from R. Starr (?) }\end{array}$ & unknown & unknown & AY948419 & n.a. & n.d. \\
\hline
\end{tabular}

n.d., not determined.

n.a., not applicable. 


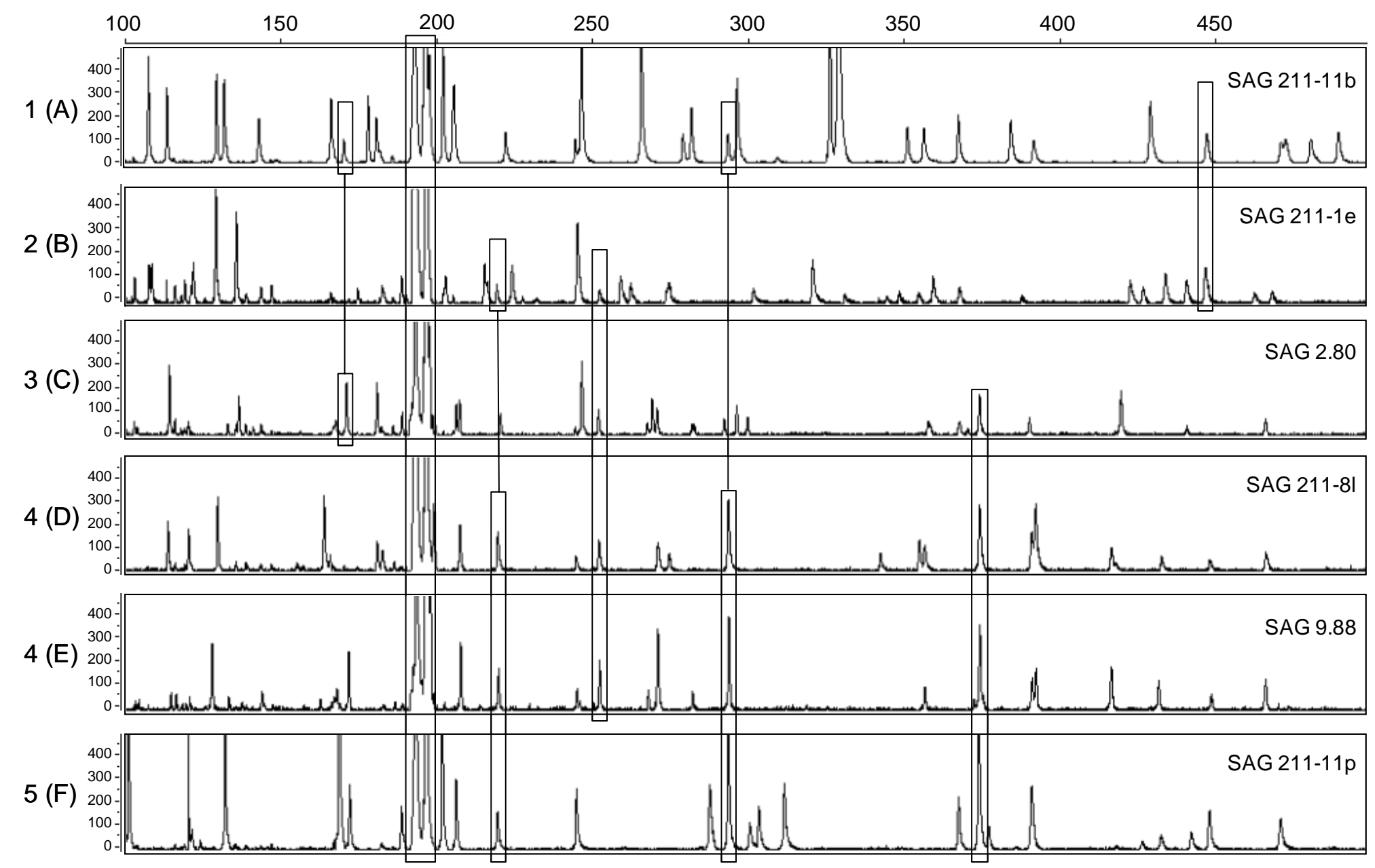

Fig. 2.2 Examples of six AFLP patterns from the five different groups that were distinguished by AFLPs among the studied strains of Chlorella vulgaris. Electropherograms from selective amplification with one primer combination, $E c 0 R I+C / M s e l+C$, and within a size range of fragments from 100 to 500 nucleotides are shown. Representative examples of homologous fragments shared among strains are within boxes. Numbers at left side indicate the corresponding clades into which the shown patterns belonged in the phylogenetic analyses (see Fig. 2.3), in brackets are corresponding ITS sequence variants. Vertical scales, relative fluorescent units; horizontal scales, size of fragment in nucleotides. 
To further investigate the relationships and genomic differences of strains within a clade, their banding patterns were compared by simple visual inspection. This drastically reduced the number of differences and eleven unique genotypes were distinguished after manual refinement (Fig. 2.3B). However, it is important to note that the manual evaluation revealed the same differences as automated evaluation for strains that were of different origins, but did not substantiate the differences that automated evaluation detected among strains of the same origin. Overestimation of differences in automatic evaluation was due to varying fragment intensities of corresponding fragments in the different samples (Fig. 2.4). Whether two strains are of the same origin was not easy to address given the poor data records available for most strains of $C$. vulgaris (Table 2.1). In addition, it is very likely that the same isolate may have been exchanged several times between early researchers of microalgal photosynthesis and metabolism and subsequently deposited in culture collections as separate strains.

Most strains for which independent origins are certain were found distributed on separated clades e.g., the 'Beijerinck isolate' in clade 1, SAG 211-11p in clade 5, and SAG 211-19 in clade 3 (Fig. 2.3A). Where differences were detected between strains of the same clade, these were (most likely to be) of independent origins, e.g. SAG strains 9.88 and 211-81 of clade 4 (Figs. 2.2, 2.3A). The three strains of clade 2 appeared to be identical after manual evaluation; they may represent the same isolate which has been accessioned by the SAG culture collection as separate strains (Table 2.1). Similarly, the pair of identical strains within clade 3 (i.e. SAG 211-11t and 211-8m) may represent the same isolate. No differences were found between the AFLP banding patterns of both duplicate strains representing the 'Beijerinck isolate' after visual inspection and, similarly, no differences were detected in all other pairs and groups of duplicate strains (Fig. 2.3B). Two groups of duplicate strains, both of uncertain origins, appeared intermixed with each other in the phylogenetic analyses (clade 4 of Fig. 2.3A), but no differences were detected after manual evaluation. To investigate the duplicate strains and others of most likely shared origins (i.e. strains of clade 3, SAG strains 211-11t and 211-8m) even more critically, selective amplifications with three additional primer combinations (EcoRI+A/MseI+0, EcoRI+C/MseI+0, EcoRI+G/MseI+0) were performed and this yielded about 150 additional fragments per strain. No differences were found with these additional fragment patterns by visual comparisons (with three replicate patterns per strain). 
A

B
SAG 211-11b

Fig. 2.3 (A) Phylogeny of the strains of Chlorella vulgaris studied based on AFLPs from automated evaluation (see text). Boxes mark pairs and groups of duplicate strains (see Table 2.1). Large numbers at internal nodes denote the five major clades resolved in the phylogenies, which are regarded as cryptic species within $C$. vulgaris (see text). A mid-point rooted maximum parsimony (MP) tree is shown. Thick lines mark internal nodes that were resolved in both MP and distance (NJ) analyses. Values above internal nodes are bootstrap values from 2000 replicates as calculated from MP (left) and NJ (right). Only values above $50 \%$ are recorded. (B) Relationships among strains within the five clades of the phylogeny from $(A)$ after manual refinement. Where identical AFLP patterns were revealed for two or more strains, the corresponding strains are given right to a triangle. (C) Distribution of ITS variants (see Fig. 2.1) among the phylogenetic lineages of the studied C. vulgaris strains. 

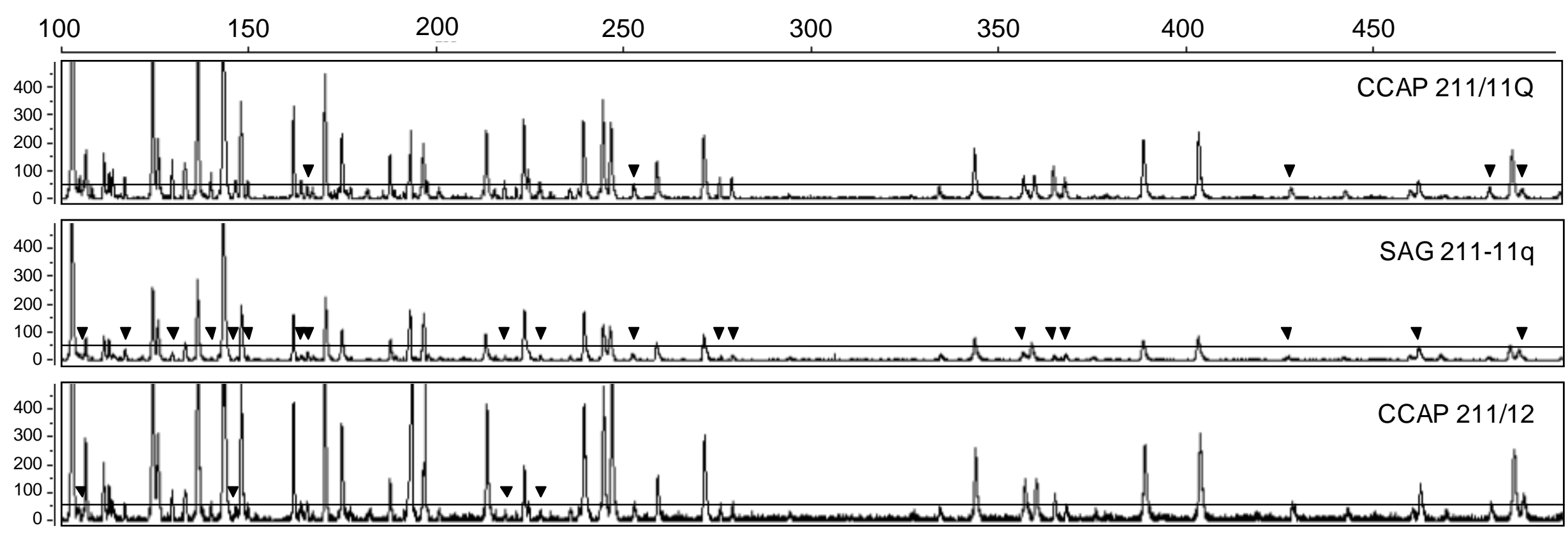

Fig. 2.4 AFLP patterns from three strains of $C$. vulgaris that are found identical after visual inspection, but intensities of fragments are different. Arrowheads denote those fragments that are shared among all three strains, but are below threshold in at least one strain. Fragments below threshold are not scored in automated band detection and, therefore, the patterns appear different in automated evaluation. Electropherograms from selective amplification with one primer combination, EcoRI+A / Msel+C, and within a size range of fragments from 100 to 500 nucleotides are shown. Vertical scales, relative fluorescent units; horizontal scales, size of fragment in nucleotides. 


\subsection{Discussion}

\subsubsection{Unique genotypes on the level of strains}

Using Chlorella vulgaris as an example, this study aimed at unravelling genomic variations among multiple strains of the same algal species as available from public culture collections. The objective was to obtain genetic signatures which allow to discriminate among various isolates of the same algal species. This is an important consideration for public culture collections of algae because they are entitled to provide the user community with pure and defined living organisms. A combination of AFLP fingerprinting with ITS rDNA sequencing, has been found very appropriate for $C$. vulgaris. We anticipate the same approaches to be successful also in other microalgae that are represented by multiple strains across several culture collections as well. Within the relatively small sample of $C$. vulgaris strains investigated here AFLPs revealed eleven unique genotypes. This impressively demonstrates that it is crucial to record which strain has been used in any experiment, or biotechnological application in order to achieve reproducibility. This is not a trivial issue and needs to be pointed out here, because many recent publications from applied research fields refer to $C$. vulgaris without providing strain numbers or identifying which culture collection it was obtained from. In a survey of the applied phycological literature from 1994 -2004, accessible through Biological Abstracts (Thomson BIOSIS, Philadelphia), more than 200 citations on C. vulgaris were obtained; however, less than 20 of these publications provided exact strain designations. Almost certainly, the number of published studies which actually used $C$. vulgaris was much higher because many investigations only referred to the genus "Chlorella" and not to a particular species. For $C$. vulgaris there may be considerable differences in quality and concentration of an exploitable valuable compound at the level of strains. Although the variation of physiological and biochemical properties of $C$. vulgaris is not known, it is likely that the genomic differences revealed by AFLPs and rDNA sequence data in this study are paralleled by phenotypic characters. Earlier studies, on a limited number of C. vulgaris strains indicated that physiological distinctions may be present within the species including excretion of different sugars, SAG 211-11b and SAG 211-1e (Kessler et al. 1991) and growth in the presence of heavy metals, SAG 211-11b and SAG 211-11f (Kessler 1986). 


\subsubsection{Test for conspecificity}

For strains CCAP 211/11F and CBS 15-2075 rDNA sequence data revealed their identities as C. sorokiniana and Parachlorella kessleri, respectively. It is probable that mislabeling, or other handling mistakes during the many years of continuous sub-culturing of these strains may have accounted for the situation. There is always the risk of human error and this is a clear disadvantage of the traditional maintenance of microalgal cultures in an actively metabolizing state (Day and Brand 2005, Lorenz et al. 2005). Cryopreservation, i.e. the storage of the cells at an ultra-low temperature (Karlsson and Toner 1996) is an effective alternative because after the initial cryopreservation, no regular handling procedures are involved, which minimizes the opportunity to mix up cultures (Day and Brand 2005). How to identify a strain as being truly $C$. vulgaris? A close relationship to the authentic strain of $C$. vulgaris, i.e. the 'Beijerinck isolate', or genetic identity with it, as revealed by ITS rDNA sequence comparisons may reliably assess the correct species identification. However, in this study no other isolate was found identical in ITS sequences to the 'Beijerinck isolate'. Strain SAG 9.88 (ITS variant E) exhibited the most similar sequence with only three positions different in ITS-2, other strains had up to six differing positions in both ITS regions (ITS variant B, Fig. 2.1). This amount of ITS sequence variation is regarded here as indicative of a 'close relationship', i.e. that the studied strains represent the same species: C. vulgaris. Considerable ITS sequence variation with the 'Beijerinck isolate' was found for species and strains that were recognized as being clearly distinct from C. vulgaris, e.g. C. sorokiniana and Parachlorella kessleri and ITS secondary structure models were needed to achieve significant alignments among the three species (Krienitz et al. 2004).

\subsubsection{Exclusion of contaminations}

AFLP is highly susceptible to contamination and foreign DNA could make two identical strains differ in a number of bands (Dyer and Leonard 2000). Could different banding patterns among $C$. vulgaris strains be obtained just because of contaminations? We can exclude contaminations by bacteria or fungi because no foreign organisms could be detected by careful microscopic examination and by using organically enriched culture medium. In addition, any precautions were taken during DNA extraction, restriction/ligation and PCR reactions to avoid contaminations (e.g. sterile conditions on a clean bench). Successive DNA extractions per strain were performed on different days with different stocks of chemicals, in most cases even from cultures of different transfers, and the number of unshared fragments between the replicates was extremely low. Independently from the AFLP 
method, there were also the ITS sequence data which revealed almost the same grouping of the strains. Viral infections may also influence the patterns and they are well known for many Chlorella species and strains (Van Etten et al. 1991). However, viral DNA should most likely introduce a considerable number of additional fragments in an AFLP pattern (cf. Chapter 6). The differences between any two banding patterns observed here cannot be explained just by the presence of additional fragments.

\subsubsection{Test for homoplasy}

There is evidence that a phylogeny inferred from a single gene does not necessarily reflect the organismal phylogeny (e.g. Lyons-Weiler and Milinkovitch 1997, Rosenberg 2002). The simultaneous analysis of many loci spread over the whole genome with AFLPs makes this method ideal for the construction of phylogenies; it has the potential to generate true species trees (Després et al. 2003). However, major concerns that have limited the use of AFLPs in phylogenetic analyses are the presence of non-homologous comigrating bands (Dowling et al. 1996) and that homoplasy obviously increases with divergence (Donaldson et al. 2000, John et al. 2004). If characters are used that evolved too fast, the amount of parallelism and reversals will reach a level at which all phylogenetic information is lost (Misof et al. 2001). Therefore, in this study we used ITS sequences to set the limits within which AFLPs can provide useful data. The AFLP pattern of one particular strain, CCAP 211/11F, had almost no shared fragments with other $C$. vulgaris strains, and the ITS sequence comparions identified this strain as phylogenetically distinct species, C. sorokiniana. We have tested the AFLP patterns from C. vulgaris for homoplasic fragments in an ongoing study. Bands of the same size which were shared among the five clades from the AFLP phylogenies have been excised and sequenced; only identical sequences have been found (Gäbler et al., in prep.). Therefore, we are confident that the AFLP-based phylogeny of $C$. vulgaris may be closer to the true organismal phylogeny than single or even multiple-gene phylogenies. 


\subsubsection{Cryptic species in Chlorella vulgaris}

Most the species of microscopic green algae described, incl. C. vulgaris, are morphological species recognized as distinct species by morphological features (Manhart and McCourt 1992, Lewis and Flechtner 2004). Molecular studies, however, revealed the existence of genetically distinct lineages within single morpho-species or among morphologically similar taxa and this is often interpreted as the presence of cryptic phylogenetic species (e.g. Taylor et al. 2000, Lewis and Flechtner 2004). Molecular differences present within a single morpho-species were found corresponding to differences in the habitat (e.g. in the green alga Scenedesmus, Lewis and Flechtner 2004) or geographical locations (e.g. in formaninifers, De Varagas et al. 1999) and then regarded as evidence for the presence of cryptic species. The molecular differences within C. vulgaris found by AFLPs in concordance with ITS sequence data may be of a similar significance for the distinction of phylogenetic cryptic species as 'multilocus sequence typing' where the concordance of multiple gene phylogenies enables to recognize species boundaries (Taylor and Fisher 2003). Therefore, we regard the five clades resolved by the AFLPs (Figs 2.2, 2.3) as five distinct cryptic phylogenetic species. This distinction is largely reflected by the various ITS variants present within C. vulgaris, i.e. each of the five major clades in the AFLP phylogeny was defined by a certain ITS variant (Fig. 2.3C). Clades 3 and 4, however, contained strains of two ITS variants. Strain SAG 9.88 (ITS variant E) in clade 4 differed in two sequence positions from variant D otherwise present in the same clade, strain SAG 2.80 (ITS variant D) in clade 3 in just one position from variant $\mathrm{C}$ of the same clade. PCR and/or sequencing errors (e.g. preferential amplification of a deviating copy from the multiple ITS gene copies) may account for these exceptions. Also, direct comparison of single gene sequences is certainly not as reliable as the multilocus-based phylogenies obtained from the AFLPs. The variable positions present in the ITS-2 regions of $C$. vulgaris strains were also mapped on the secondary structure model presented by Krienitz et al. (2004) in order to check for the presence of twosided compensating base changes (CBCs). These have been found as a measure for the distinction of biological species of algae at the molecular level (e.g. Coleman 2000). When two-sided CBCs occur between two strains of an alga capable of sexual reproduction, no successful interbreeding could be observed. In C. vulgaris sexual reproduction is not known. No two-sided CBCs were found among the studied strains of $C$. vulgaris (e.g. there was only a single one-sided CBC among the four variable positions of ITS-2) which may indicate that the genomic differences found are below the level of a biological species. 


\subsubsection{Tracing the origin of strains}

The AFLP method was found very appropriate to unequivocally identify a certain isolate of $C$. vulgaris and to trace the origins of strains with great resolution. Among $C$. vulgaris strains where only little ITS rDNA sequence variation was present, AFLPs provided a much higher resolution. Strains representing different isolates, i.e. those which where of different (independent) origins were well distinguished by a large number of AFLP fragments (Fig. 2.2) and were clearly resolved as distinct lineages in the AFLP-based phylogenies (Fig. 2.3). The suitability of AFLPs as sensitive tools to clearly discriminate among isolates and to trace the origin of strains of freshwater microalgal species has been further substantiated by preliminary results we obtained from a variety of green algae which includes Scenedesmus obliquus, Chlorogonium spp., Coccomyxa spp. and Klebsormidium flaccidum (J. Müller unpubl.). This is in agreement with previous studies where AFLPs have been found well suited to resolve genetic distances among specimens of macroalgae from geographically distant regions (e.g. Mannschreck et al. 2002, Erting et al. 2004). AFLPs have been proven reliable tools also in bacteria taxonomy (Arias et al. 2004, Burke et al. 2004) for the delineation of species (identification) and for the discrimination of strains ('typing', Savelkoul et al. 1999). Over many decades strains of $C$. vulgaris have served as a model organism for plant physiological and biochemical studies since the pioneering work of Warburg (1919) and Pirson (1937, 1994). Strains of C. vulgaris of various origins may have been used by these early workers which they and their successors may have also frequently exchanged and which then later were deposited as separate strains at different public culture collections. Both strains 211-11t and 211-8m represent the same isolate, which was used in the pioneering studies of O. Warburg and others, but which later was deposited at the SAG culture collection at two different times. Similarly, SAG 211-1e and SAG 30.80 represent the same isolate which was evidently used in two independent laboratories (H. Tamiya, Tokyo, and H. Senger, Marburg). The strains from both laboratories were later accessioned from the SAG culture collection at different times. The AFLP data strongly suggest that both strains represent the same, isolated by E.G. Pringsheim in 1939 (Table 2.1). Strains 211-11q and 211-12 from SAG as well as 211/11Q and 211/12 are among the oldest available strains of C. vulgaris and are almost certainly the same isolate, isolated by one of the pioneers in algal cultivation, E.G. Pringsheim, V. Czurda, or R. Chodat (Day et al. 2004, Mollenhauer 2004). The isolate was probably maintained in duplicate at two independent laboratories (Prague and Geneva) and it is still maintained in duplicate at three independent culture collections, CCAP, SAG and UTEX. 


\subsubsection{Duplicate strains}

It is remarkable that no genomic differences have been found in pairs and groups of duplicate strains. The investigated $C$. vulgaris strains from the SAG and UTEX culture collections have been continuously maintained without cryopreservation, whereas the strains from CCAP have been stored in a cryopreserved state at $-196^{\circ} \mathrm{C}$ since the 1970 's. Continuous sub-culturing of an algal strain over thousands of generations under the selective pressure of laboratory culture is likely to favor genetic change due to selective pressure and/or genetic drift (Brand and Diller 2004). This could lead to genetic differences between strains maintained at different culture collections. A re-isolation of clonal cultures from strains UTEX 30 and UTEX 265, which was performed recently because they were contaminated (J. Brand, pers. comm.) would have provided another possibility for genetic drift. Although cryopreservation provides stability against changes in the genetic composition of cultures over time, one could also argue that the various steps of the cryopreservation procedure causes considerable stress to algal cells, e.g. toxicity of the cryoprotectant, osmotic shock, and intracellular ice formation (Day et al. 2000) and this may potentially lead to genomic alterations. However, with AFLPs no genetic effects due to different maintenance methods could be detected here. Even careful visual inspection of additional AFLP patterns did not reveal any differences. However, it may well be that the genomic effects were too small in such a robust alga as $C$. vulgaris to be detected by AFLPs. In other algae with a higher susceptibility to various cryogenic treatments differences may be detected. This is currently being investigated within the European research project, COBRA (Day et al. 2003).

\subsubsection{Evaluation and reproducibility of AFLP patterns}

Variation in fragment intensities has been identified as a main source of errors that can lead to artificial differences in both, automated and manual AFLP pattern evaluation (Bonin et al. 2004). Intensities of a number of bands within a fragment pattern may not be reproducible for various reasons (Savelkoul et al. 1999, Donaldson et al. 2000). In this study we tried to compensate for this effect in automated evaluations by the construction of a consensus pattern from three replicate AFLP reactions. However, as visual examination demonstrated, this could not completely eliminate errors due to fragment intensities. Although automated and manual evaluation of the AFLP patterns were largely congruent, the automated evaluation appeared to have overestimated differences. This may be due to that automated fragment evaluation was affected by differences in the intensities of the fragments (peak heights) in the AFLP patterns. For example, the close visual inspection showed that the AFLP patterns (three 
replicates) from the two duplicate strains, SAG 211-11q and CCAP 211/11Q, were identical, but the intensity of fragments was generally lower in SAG 211-11q than in CCAP 211/11Q (Fig. 2.4). This resulted in a considerable number of fragments being below the threshold and, therefore, were not detected by automated evaluation. For another strain, CCAP 211/12, the AFLP pattern was found identical with that of CCAP 211/11Q and SAG 211-11q. However, there were differences in peak heights and this lead several fragments of CCAP 211/12 being not detected by automated evaluation. Generally, a fixed threshold value is defined to discriminate DNA fragments from oscillations of the background noise. This interpretation procedure can lead to erroneous peak detections especially if the overall signal is low and if a high number of fragments is shared by two samples. Therefore we tried to define variable threshold values as percentage values of the average peak intensity or of the highest peak intensity. This did not improve the quality of the interpretation. Visual comparison is not feasible with a larger number of samples as it is extremely time-consuming (De Haan et al. 2002) and has been found as a major source of errors in AFLP evaluation due to its subjective nature (Bonin et al. 2004). For the construction of phylogenies from AFLP patterns an automated evaluation is most accurate, but it needs manual correction when two patterns are rather similar.

In order to achieve high reproducibility of the AFLP technique it is essential to use high molecular and pure DNA and this was found the most critical factor in the present study. Therefore, DNA extractions were performed in two replicates here. Furthermore, in order to minimize possible effects from PCR amplifications, all AFLP reactions were done three times per strain. The reproducibility of AFLP was also tested for an extended sample of microalgae of different morphology and taxonomy in the present thesis (Chapter 5). Reactions were regarded as satisfactory when selective amplifications still yielded a reasonable number of fragments of high intensity above $300 \mathrm{bp}$. The number of fragments with larger peak heights that was not shared among the three replicate reactions was extremely low. The percentage of non-reproducible AFLP fragments in this study was found to be below 3\% which is comparable to other studies which used other organisms (e.g. Donaldson et al. 1998, Chavarriaga-Aguirre et al. 1999, Hauben et al. 1999, John et al. 2004). Mainly fragments with small peak heights close to background noise which were likely due to artifacts in the selective amplification, (Bonin et al. 2004) were found less reproducible. A high reproducibility (90-98\%) of AFLP fragments has been demonstrated between different laboratories (Jones et al. 1997), but it has been criticized that this study used the same original DNA sample in the replicates (Donaldson et al. 2000). Therefore a comparison of AFLP 
reactions performed by different laboratories starting with DNA extractions using the same strain is needed to provide more sound data on the reproducibility of the AFLP method.

\subsection{Conclusion}

To conclude, C. vulgaris was found a proper example for an alga where considerable genomic variation among the multiple strains available from public culture collections is present. This is normally obscured by morphological species recognition. We hope to have demonstrated here that a combined approach, AFLP genotyping and ITS rDNA sequence comparisons, are most appropriate to unravel genomic diversity below the level of species. AFLPs allow the clear distinction of unique genotypes, i.e. to reveal genetic signatures by fingerprinting, and to trace the origin of isolates. ITS sequences are required to check for conspecificity in comparisons with authentic culture material and to set the limits within which the usage of the highly sensitive AFLPs is meaningful. The approach shown here may help service culture collections to preserve biodiversity more efficiently, that is to avoid unnecessary duplication and to select unique genotypes. 
3 Detection of genetic alterations among pigment mutants of Parachlorella kessleri induced by different mutagenesis techniques (UV light, $x$-ray, radioisotope) based on amplified fragment length polymorphism (AFLP)

\begin{abstract}
3.1 Abstract
AFLP is a DNA fingerprinting method that has previously been shown to be capable of distinguishing between different isolates of the same species. Here it was tested if this technique is also sensitive enough to discriminate at the next level of resolution, namely between mutants of the same isolate. Pigment mutants of the strain Parachlorella kessleri SAG 211-11h investigated in this study were induced by UV light, radioisotope treatment or x-ray irradiation in the 1950s and 1960s. The amount of mutations induced by these mutagenesis techniques is not known, but all strains exhibit different phenotypic properties, especially obvious in their pigment composition or differences in carotenoid biosynthesis. AFLP analyses revealed that the three $\mathrm{x}$-ray mutants were mislabeled. Subsequent sequence analyses of the 18S rDNA showed that they do not present members of the genus Parachlorella, but rather exhibit high similarity to Chlorella mirabilis. In AFLP analyses, no genetic differences were found among them, whereas clear differences were obtained for the UV light and radioisotope mutants. Of the 285 fragment positions studied, between two and eight positions were found to be variable. Replication of banding patterns was found to be an adequate tool to identify non-reproducible fragments and improved the accuracy and reliability of the AFLP analyses. It was apparent, that three mutations were present in the majority of all mutants. It is therefore possible that these mutations were generated from a locus in the genome that is correlated with carotenoid biosynthesis.
\end{abstract}

\title{
3.2 Introduction
}

It has been previously demonstrated that AFLP was capable of discriminating isolates of the same species (Chapter 2). The aim of the present study was to investigate if the AFLP technique is also sensitive enough to discriminate at the next level of resolution, namely between mutants of the same isolate. Twelve pigment mutants of the wildtype Parachlorella kessleri SAG 211-11h (Chlorophyta, Trebouxiophyceae, Fig. 1.3b) from the SAG culture collection were investigated. These mutants were induced by UV light (Fig. 1.3c), radioisotopes or x-ray irradiation in the 1950s and 1960s (e.g. Claes 1954, Schwarze and Frandsen 1960), and it is not known how many parts of the mutant genomes were influenced by mutagenesis. AFLP banding patterns of the x-ray mutants were found to be significantly 
different from the wildtype and the other mutants. 18S rDNA sequence analyses confirmed that these mutants are not members of the genus Parachlorella and were mislabeled; results of their AFLP analyses are therefore discussed separately.

The wildtype strain SAG 211-11h was formerly referred to as Chlorella vulgaris but was later reassigned into Chlorella kessleri (Fott and Nováková 1969). Today this species is referred to as Parachlorella kessleri (Krienitz et al. 2004). While some publications are available about investigations of x-ray and radioisotope mutants, none could be found about UV light mutants.

The SAG culture collection obtained the three $\mathrm{x}$-ray mutants from $\mathrm{H}$. Claes from Tübingen in 1975 as pigment mutants of SAG 211-11h. The strain SAG 211-11h was sent from SAG to H. Claes in 1953. These three mutants exhibit blocks in the synthetic pathway of the carotenoids and a more or less completely blocked chlorophyll synthesis (Claes 1954). A series of publications on the biosynthesis of carotenoids of one of these mutants with the number 5/520, which corresponds to the SAG number 16.80, appeared in the journal Zeitschrift für Naturforschung in the 1950s (Claes 1956, 1957, 1958a, 1959). Further publications dealt with the interactions of chlorophyll and carotenes and other biochemical analyses of this mutant (Claes and Nakayama 1959, Claes 1960, 1961, 1966). Only two publications are available where all three mutants are compared (Claes 1958b, 1962). These publications also report on differences in the carotenoid synthesis of these three mutants. No further information is available about the amount of genetic differences in comparison to the wildtype strain.

Less information is available for the other mutants. The four radioisotope mutants were obtained from P. Schwarze from Köln-Vogelsang as mutants of SAG 211-11h (SAG 211-11h/20 and SAG 211-11h/9 were obtained in 1962 and SAG 9.80 and SAG 8.80 in 1971). Their mutagenesis and some phenotypic attributes (e.g. colour, chlorophyll content) are described in Schwarze and Frandsen (1960), but this publication does not refer to any strain numbers and thus reassignment of these mutants to SAG strain numbers was not possible. The ability to three of these four mutants, SAG 9.80, SAG 8.80 and SAG 211-11h/9, to excrete formic acid was further investigated and small differences were found (Schwarze 1965b). A third publication dealt with the chlorophyll accumulation in correlation with amino acids of the mutant 113 (Schwarze 1965a). This strain number can also not be reassigned either to one of the four radioisotope mutants or any of the five UV light mutants. The UV light mutants were obtained from P. Schwarz in 1971 and 1978. All available information on these mutants is listed in Table 3.1, no further details or publications about them are available. 
With respect to the observable phenotypic differences between all mutants and the small differences found between some of them by H. Claes and P. Schwarz, it is likely that genetic alterations are present in the genomes of the mutants. The AFLP technique was therefore employed here to try if these mutations can be detected and if there are any differences in the amount of mutations in correlation to the technique of mutagenesis.

\subsection{Material and Methods}

\subsubsection{Investigated strains, DNA extraction, AFLP and 18S rDNA analyses}

All strains were obtained from the SAG culture collection and are listed in Table 3.1 and Table 3.3. DNA extraction and AFLP reactions were carried out as described for Chlorella vulgaris in Chapter 2 and the following three primer combinations were used in selective amplification: $\quad E c o \mathrm{RI}+\mathrm{A} / M s e \mathrm{I}+\mathrm{C}, \quad E c o \mathrm{RI}+\mathrm{C} / M s e \mathrm{I}+\mathrm{C}$ and $E c o \mathrm{RI}+\mathrm{A} / M s e \mathrm{I}+\mathrm{G}$. Reproducibility tests of banding patterns were performed by four independent replications of the complete AFLP technique per strain. Three replications were conducted using a first DNA extraction and the fourth replication using a second. All AFLP banding patterns were manually evaluated using the software GenScan from 100 to 500 bp. Differences in patterns between two samples were only counted if they were reproducible, i.e. if the differing fragment was present or absent in all four replications (cf. Chapter 5.3.4). The resulting differences were translated into a binary matrix, with zero representing an absent fragment and one a present fragment. A binary matrix of twelve positions was obtained and a UPGMA (Unweighted Pair Group Method with Arithmetic Mean, Sneath and Sokal 1973) cluster analysis with Nei-Li distance settings was performed using the software PAUP* V4.0b10 (Swofford 2001). Due to the small size of only twelve characters it was not deemed necessary to perform further cladistic analyses, e.g., as had been conducted for the 490 characters obtained for Chlorella vulgaris (Chapter 2.3.5). The 18S region of the rDNA was amplified, sequenced, evaluated and blasted as described in Chapter 2 for Chlorella vulgaris.

\subsection{Results and Discussion}

\subsubsection{Genetic differences between wildtype and mutants of $P$. kessleri}

Manual comparisons of all banding patterns of the wildtype, the UV light- and radioisotope mutants (Table 3.1) revealed 285 fragment positions and twelve positions were found to be variable (polymorphic). One of these polymorphic positions is denoted with asterisks in Fig. 3.1. Comparison of these twelve differing fragments in a binary matrix 
showed that not all mutations were randomly distributed over the genome (Table 3.2). In the binary matrix a zero denotes a fragment absent at a certain position and a one a fragment that is present. Three fragment positions were obtained where seven or eight of the nine mutants lack a fragment that was present in the wildtype (Table 3.2, fragment positions two, three and nine). These three differing fragment positions were present in all UV light mutants as well as in three or two of the radioisotope mutants. It is therefore possible that these fragments were absent in the wildtype due to mutations in a locus of the genome that is, for example, correlated with carotenoid biosynthesis. A correlation with a locus in the genome responsible for pigments or photosynthesis can also be expected for the fragment positions four, eleven and twelve where six, five or four of the nine mutants lack a fragment present in the wildtype. However, there were, as it can be expected for non-specifically introduced mutations, also unique polymorphisms for certain mutants that were not present in any of the other mutants, e.g. fragment position one for SAG 211-11h/9 or positions seven for SAG 14.80 (Table 3.2). In addition, an attempt was made to excise and sequence two of the three fragments from the wildtype that were not present in the majority of the mutants (fragment positions two of 190 bp and three of $225 \mathrm{bp}$ ), but the sequencing reaction failed for unknown reasons (pers. comm., S. Gäbler). Due to time constraints this experiment could so far not be replicated, but it would be an excellent possibility to obtain the sequence information from this locus of the genome. Few microalgal genome sequences are available in public databases, but if this locus is correlated with pigment biosynthesis there might be sufficient sequences available from higher plants to enable reasonable comparisons.

\section{Correlation of genetic differences at the phenotypic level}

The similarities or dissimilarities between mutants and the wildtype were displayed in a UPGMA dendrogram constructed on the basis of the twelve positions of the binary matrix (Fig. 3.2). In this dendrogram, the mutant SAG 9.80 was most distant from all other samples, while the UV light mutants were found to be more similar to each other than to the other mutants and formed a separated clade. In general it seemed that UV light had a more uniform influence on the algal cells than radioisotopes. Banding patterns of the wildtype were similar to the mutants SAG 211-11h/20 and SAG 8.80, which in turn were identical in their banding patterns. The genetic differences obtained from AFLP analyses could be correlated with the phenotypic differences in the radioisotope mutants SAG 9.80, SAG 8.80 and SAG 211-11h/9

found by P. Schwarz (colour and excretion of formic acid, cf. Chapter 3.2). The UV light mutants SAG 12.80, 13.80 and 14.80 showed identical phenotypic attributes (Table 3.1). This 
could be corroborated for SAG 12.80 and 13.80 by the AFLP analyses where these two strains were most similar to each other, while SAG 14.80 was more similar to SAG 11.80 (Fig. 3.2). Unfortunately, no further information was available about phenotypic properties of these UV light mutants to correlate them with the results obtained here at the molecular level.

The differences found in this study for the pigment mutants of $P$. kessleri were higher than the differences found between mutants of Dunaliella salina induced by the mutagenic chemical EMS (Chapter 4). Despite analysis of 800 AFLP fragments in Dunaliella, no differences were found, whereas here, a total of twelve differing fragment positions were obtained in less than 300 investigated AFLP fragments. These findings can be explained by the different strength of mutagenic effect of the different techniques of mutagenesis. EMS, for instance, is known to lead to fewer genomic mutations than UV light (Lawrence 2002 and cf. Chapter 4.5.4).

\subsubsection{Test for reproducibility of the banding patterns}

A fragment position was only counted as polymorphic if the presence or absence of a fragment was reproducible in all four replicated AFLP banding patterns per strain. Arrowheads in Fig. 3.1 denote a non-reproducible fragment that was present in all four replicates of the wildtype but only in three replicates of the mutant. This position was therefore not counted as a difference between both strains. It is evident from this example that replicates of the AFLP banding patterns are necessary to avoid the evaluation of nonreproducible fragments. These non-reproducible fragments likely represent artefacts rather than genetic alterations (cf. Chapter 5.5.2). The usefulness of these replications was for example also shown in a reproducibility test of AFLP banding patterns for 23 different species of microalgae (Chapter 5). It is obvious that false differences would be evaluated if only one AFLP banding pattern per strain is compared. One can minimize the influence of this error source by comparing replicates per strain. In this way non-reproducible fragments are identified and it is more likely that only 'true' and reproducible fragments are evaluated. An example for a 'true' (reproducible) polymorphic position is denoted in Fig. 3.1 with an asterisk. 
Table 3.1 Overview of the mutants and the corresponding wildtype strain used in AFLP analyses, technique of mutagenesis, available information about the phenotype and author of mutagenesis.

\begin{tabular}{|c|c|c|c|c|}
\hline Strain & $\begin{array}{c}\text { Wildtype / } \\
\text { mutant }\end{array}$ & & Phenotype & Author \\
\hline SAG 211-11h & wildtype & green & wildtype & \\
\hline SAG 10.80 & UV light & white & - & P. Schwarz \\
\hline SAG 11.80 & UV light & orange/brown & - & P. Schwarz \\
\hline SAG 12.80 & UV light & yellow & $\begin{array}{l}\text { produces no chlorophyll } \\
\text { in darkness with sugar }\end{array}$ & P. Schwarz \\
\hline SAG 13.80 & UV light & yellow & $\begin{array}{l}\text { produces no chlorophyll } \\
\text { in darkness with sugar }\end{array}$ & P. Schwarz \\
\hline SAG 14.80 & UV light & yellow & $\begin{array}{l}\text { produces no chlorophyll } \\
\text { in darkness with sugar }\end{array}$ & P. Schwarz \\
\hline SAG 211-11h/20 & $\begin{array}{l}\text { radioisotopes } \\
\mathrm{P}^{32} \mathrm{~S}^{35}\end{array}$ & yellow & $\begin{array}{c}\text { produces only lutein and } \\
\text { antheraxanthin }\end{array}$ & P. Schwarz \\
\hline SAG 211-11h/9 & $\begin{array}{l}\text { radioisotopes } \\
\mathrm{P}^{32} \mathrm{~S}^{35}\end{array}$ & yellow & - & P. Schwarz \\
\hline SAG 8.80 & $\begin{array}{l}\text { radioisotopes } \\
\mathrm{P}^{32} \mathrm{~S}^{35}\end{array}$ & orange/brown & $\begin{array}{l}\text { produces only } \\
\text { xanthophylls }\end{array}$ & P. Schwarz \\
\hline SAG 9.80 & $\begin{array}{l}\text { radioisotopes } \\
\mathrm{P}^{32} \mathrm{~S}^{35}\end{array}$ & $\begin{array}{l}\text { white, old } \\
\text { culture } \\
\text { reddish }\end{array}$ & $\begin{array}{c}\text { produces only chlorophyll } \\
\text { a and only a bit of } \\
\text { chlorophyll b }\end{array}$ & P. Schwarz \\
\hline
\end{tabular}

Table 3.2 Binary matrix of AFLP evaluation. Only polymorphic positions are shown. Grey background denotes positions that are different (diff.) from the wildtype strain (WT).

\begin{tabular}{|c|c|c|c|c|c|c|c|c|c|c|c|c|c|c|}
\hline \multirow{2}{*}{\multicolumn{2}{|c|}{ Wildtype/mutant SAG no. }} & \multicolumn{12}{|c|}{ fragment position } & \multirow[t]{2}{*}{ diff. to WT } \\
\hline & & $1^{\mathrm{a}}$ & $2^{\mathrm{a}}$ & $3^{a}$ & $4^{\mathrm{a}}$ & $5^{a}$ & $6^{\mathrm{b}}$ & $7^{b}$ & $8^{\mathrm{b}}$ & $9^{\mathrm{b}}$ & $10^{\mathrm{b}}$ & $11^{\mathrm{b}}$ & $12^{b}$ & \\
\hline WT & $11 \mathrm{~h}$ & 1 & 1 & 1 & 1 & 0 & 1 & 0 & 1 & 1 & 1 & 1 & 1 & \\
\hline \multirow{5}{*}{ UV-light } & 10.80 & 1 & 0 & 0 & 0 & 1 & 1 & 0 & 1 & 0 & 1 & 1 & 1 & 5 \\
\hline & 11.80 & 1 & 0 & 0 & 0 & 0 & 1 & 0 & 1 & 0 & 0 & 0 & 1 & 6 \\
\hline & 12.80 & 1 & 0 & 0 & 0 & 0 & 1 & 0 & 1 & 0 & 1 & 0 & 0 & 6 \\
\hline & 13.80 & 1 & 0 & 0 & 1 & 0 & 1 & 0 & 1 & 0 & 1 & 0 & 0 & 5 \\
\hline & 14.80 & 1 & 0 & 0 & 0 & 0 & 1 & 1 & 1 & 0 & 1 & 0 & 1 & 6 \\
\hline \multirow{4}{*}{ radioisotopes } & 8.80 & 1 & 0 & 0 & 1 & 0 & 1 & 0 & 1 & 1 & 1 & 1 & 1 & 2 \\
\hline & $11 \mathrm{~h} / 20$ & 1 & 0 & 0 & 1 & 0 & 1 & 0 & 1 & 1 & 1 & 1 & 1 & 2 \\
\hline & 9.80 & 1 & 0 & 0 & 0 & 0 & 0 & 0 & 0 & 0 & 1 & 0 & 0 & 8 \\
\hline & $11 \mathrm{~h} / 9$ & 0 & 1 & 1 & 0 & 0 & 1 & 0 & 1 & 0 & 1 & 1 & 0 & 4 \\
\hline $\begin{array}{l}\text { No. of mutants } \\
\text { diff. to the WT }\end{array}$ & & 1 & 8 & 8 & 6 & 1 & 1 & 1 & 1 & 7 & 1 & J & 4 & \\
\hline
\end{tabular}

${ }^{\mathrm{a}}$ Fragment position obtained with EcoRI+C/MseI+C (1 refers to 175 bp, 2 to 190 bp, 3 to 225 bp, 4 to 325 bp and 5 to $480 \mathrm{bp})$.

${ }^{\mathrm{b}}$ Fragment positions obtained with EcoRI+G/MseI+C (6 refers to $180 \mathrm{bp}, 7$ to $200 \mathrm{bp}, 8$ to 215 bp, 9 to $340 \mathrm{bp}, 10$ to $370 \mathrm{bp}, 11$ to $419 \mathrm{bp}$ and 12 to $420 \mathrm{bp}$ ). 
A 120

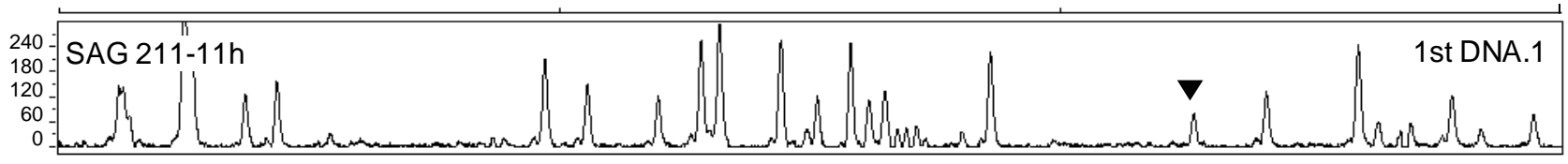

B

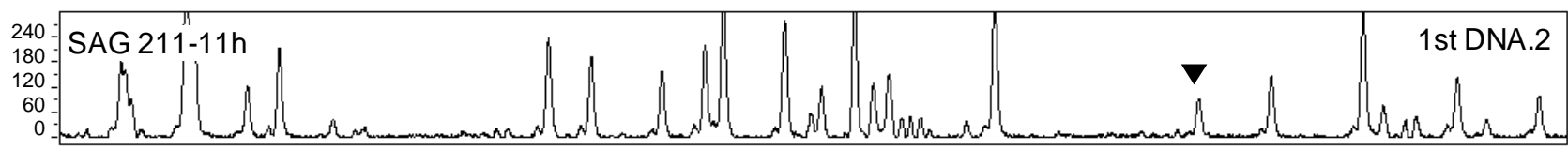

C 120

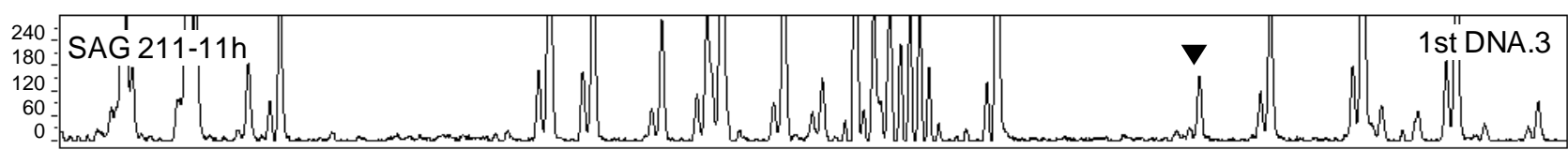

D 12

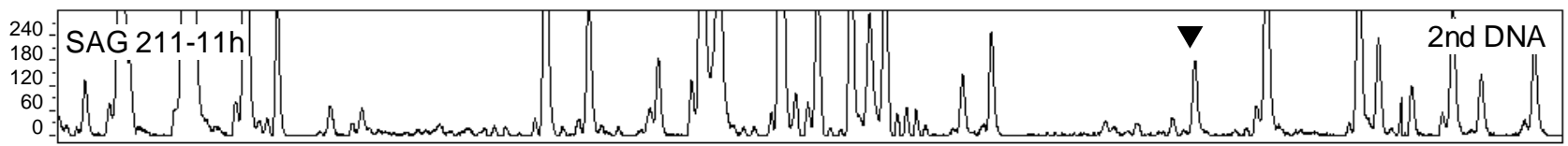

E

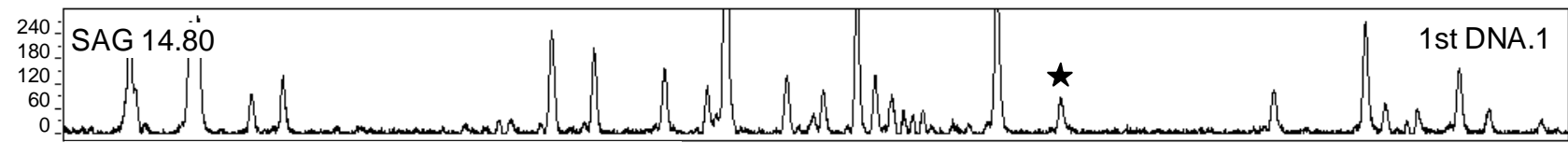

$\mathrm{F}$
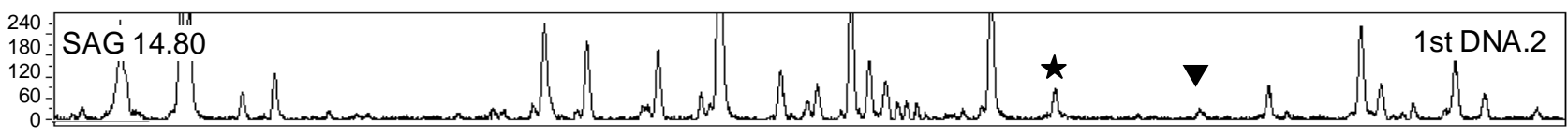

G 120

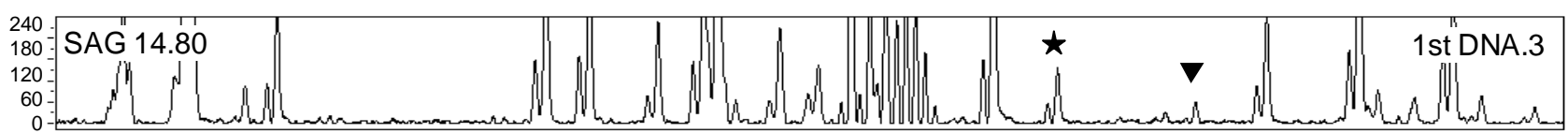

240
180

H $\quad 120$

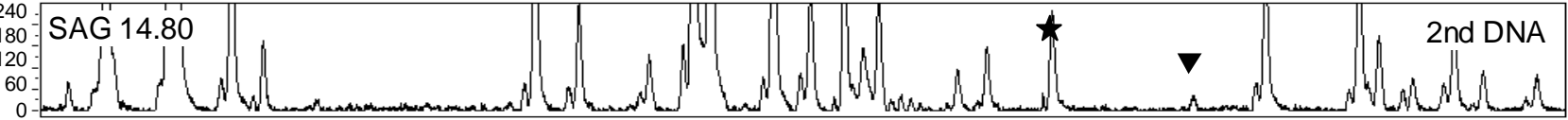

Fig. 3.1 Comparison of wildtype strain and UV light mutant SAG 14.80 and test for reproducibility. Examples for AFLP patterns of the wildtype SAG 211-11h

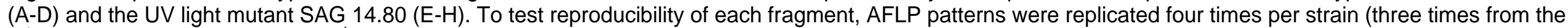

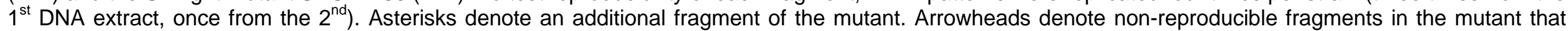
were not counted as a difference in comparison to the wildtype. Electropherograms of primer combination EcoRI+G/Msel+C. Vertical scales, relative fluorescent units; horizontal scales, size of fragment in nucleotides. 


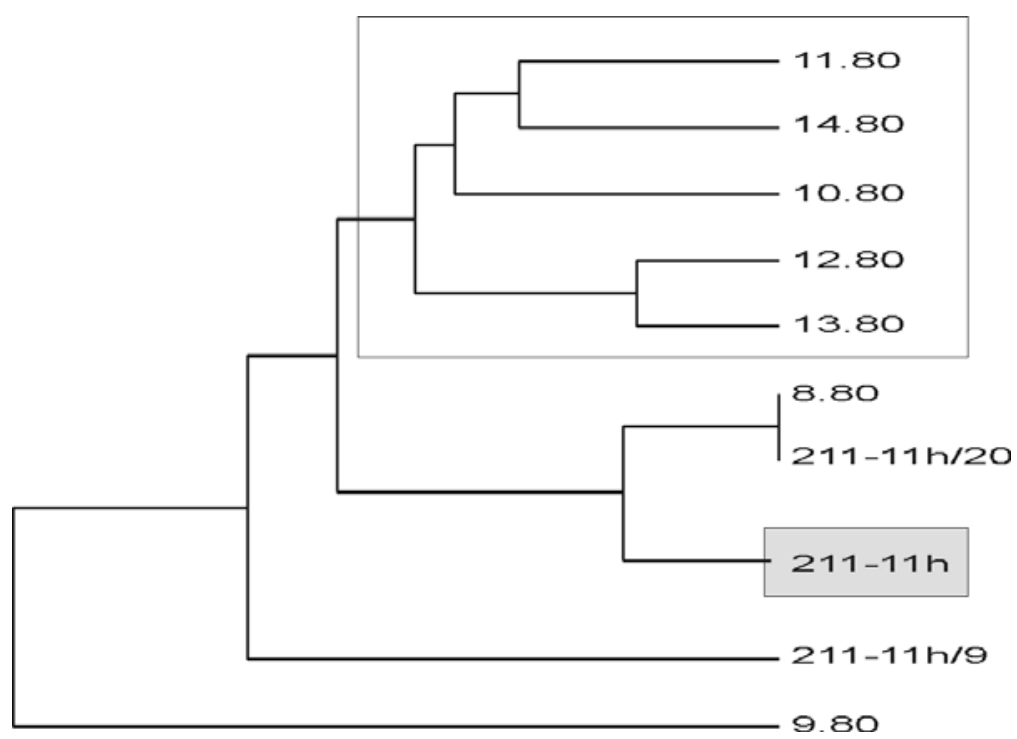

Fig. 3.2 UPGMA dendrogram (Nei-Li distance metric) generated from the binary matrix derived from AFLP analyses of the wildtype strain (within the grey box), the UV light mutants (inboxed) and the radioisotope mutants. Labels display the SAG number.

\subsubsection{Genetic differences within the x-ray mutants}

Since AFLP patterns of different genera are not comparable (cf. homoplasy, Chapter 2.5.4) the x-ray mutants (Table 3.3) were excluded from comparisons with the other mutants and were only compared among each other.

Table 3.3 Overview of the x-ray mutants, phenotypic attributes and author of mutagenesis.

\begin{tabular}{ccccc}
\hline \hline Strain & $\begin{array}{c}\text { Wildtype } / \\
\text { mutant }\end{array}$ & Phenotype & Author \\
\hline SAG 15.80 & x-ray & $\begin{array}{c}\text { colourless in } \\
\text { darkness }\end{array}$ & produces no xanthophylls & H. Claes \\
\hline SAG 16.80 & x-ray & $\begin{array}{c}\text { colourless in } \\
\text { darkness }\end{array}$ & $\begin{array}{c}\text { no xanthophylls, almost no } \\
\text { chlorophyll }\end{array}$ & H. Claes \\
\hline SAG 17.80 & x-ray & $\begin{array}{c}\text { colourless in } \\
\text { darkness }\end{array}$ & $\begin{array}{c}\text { no carotine and xanthophylls, } \\
\text { almost no chlorophyll }\end{array}$ & H. Claes \\
\hline
\end{tabular}

Manual comparison of their AFLP banding patterns revealed 261 fragment positions and no differences were found. Part of the identical banding patterns obtained for these three mutants is shown in Fig. 3.1. The differences in carotenoid biosynthesis between these mutants (cf. Chapter 3.2) could therefore not be correlated with differences on the molecular level using AFLP. Extensive AFLP analyses using additional primer- and maybe also enzyme combinations could be conducted to further search for genetic alterations. With regard to the phenotypic differences and the technique by which the mutants were induced, it is likely that genetic alterations are present. But one must keep in mind that these genetic alterations can represent very small mutations or even point mutations, which only have a small chance of 
being detected using fingerprinting methods. It is however also not known when these strains were mislabeled and if they really represent the cultures used in the experiments that were published by H. Claes.

\subsubsection{Identification of the x-ray mutants}

AFLP banding patterns of the three x-ray mutants were found to be uniformly and considerably different from the wildtype strain (Fig. 3.3).

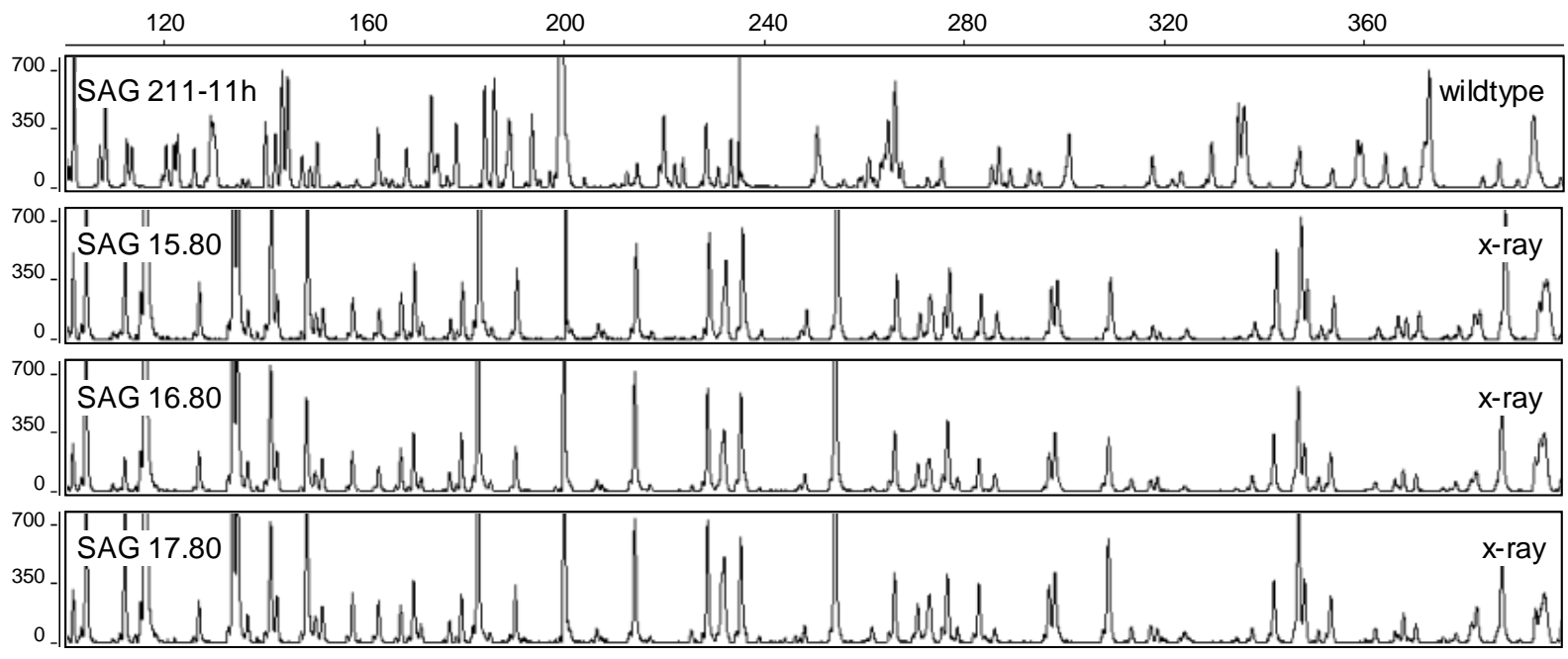

Fig. 3.3 Example of AFLP banding patterns for the wildtype strain Parachlorella kessleri SAG 211-11h and the x-ray mutants SAG 15.80, SAG 16.80 and SAG 17.80. Electropherograms of primer combination $\mathrm{EcoRI}+\mathrm{C} / \mathrm{Msel}+\mathrm{C}$. Vertical scales, relative fluorescent units; horizontal scales, size of fragment in nucleotides.

18S rDNA was therefore sequenced from one of these x-ray mutants (SAG 16.80) to test whether these mutants really represent Parachlorella kessleri. Sequencing reactions resulted in a partial sequence of 1666 nucleotides. Manual comparison of this sequence with the 18S rDNA sequence of the authentic type strain of Parachlorella kessleri SAG 211-11g (accession number X56105) revealed 76 different positions (4.5\%). The 18S rDNA region is a molecular marker molecule to assess phylogenetic relationships at and above the genus level (Huss and Sogin 1990, Krienitz et al. 2004). For strains of the same species no differences among 18S rDNA sequences are therefore expected. The 76 sequence differences found between the mutant SAG 16.80 and SAG 211-11g revealed that SAG 16.80 cannot belong to P. kessleri. For identification of this strain, the $18 \mathrm{~S}$ rDNA from SAG 16.80 was used to query the sequence database on the NCBI BLAST page (www.ncbi.nlm.nih.gov/BLAST). The BLAST search results showed Chlorella mirabilis Andreya 748-I (99\% identical, accession number X74000), Chlorella sphaerica SAG 11.88 (97\% identical, accession number AJ416105) and Stichococcus sp. MBIC10465 (97\% identical, accession number AB183601) 
as the most similar organisms. All of these strains belong the "Prasiola-clade" of the Trebouxiophyceae (Karsten et al. 2005). It was accordingly evident that the x-ray mutants do not represent mutants of SAG 211-11h and do not belong to the Chlorellaceae sensu Krienitz et al. (2004) to which P. kessleri belongs; these mutants were mislabeled. There are some cases known where microalgae were mislabeled which might be explained by the fact that microalgae lack unique morphological characters for an easy identification in a culture test tube (cf. Chapter 4.5.3). It is possible that the wildtype of these mutants is the strain Chlorella mirabilis SAG 211-11r which was also used in physiological studies in the 1950s (e.g. Kessler 1953) and obviously has a very similar strain number to SAG 211-11h. 


\title{
4 Applicability of amplified fragment length polymorphism for genetic characterization of non-axenic strains of microalgae: a case study using mutants of Dunaliella salina
}

\begin{abstract}
4.1 Abstract
The fingerprinting technique AFLP is a recently developed approach tailored to genetic investigations below the species level. In the present study it was used for the genetic characterization of non-axenic mutants of the microalga Dunaliella salina. D. salina produces large amounts of carotenoids and is thus a valuable organism for a wide variety of biotechnological applications. Normally, AFLP analyses are not reliable for contaminated organisms, because the resulting fragments cannot be assigned with certainty to the organism in study or to the contamination. However, it was demonstrated here that it is actually possible to use AFLP to investigate non-axenic cultures. This was achieved by comparing banding patterns generated from cultures with differing ratios of Dunaliella and contaminating bacteria and/or fungi. Out of the 865 fragment positions obtained, 22 polymorphic positions were identified as contaminant-specific fragments and, therefore, excluded from further phylogenetic analyses. This was made possible by the fact that the same contamination was present in all strains. Application of this procedure revealed no differences between EMSinduced mutants (VD 6, VD 72 and VD 107) and the wildtype D. salina CCAP 19/30. In addition, the axenic duplicate of the wildtype (DCCBC 15) and another strain of $D$. salina (DCCBC 2) were analysed. In combination with ITS rDNA analyses it was concluded that DCCBC 15 and CCAP 19/30 are not duplicate strains and that DCCBC 2 is more similar to CCAP 19/30.
\end{abstract}

\subsection{Introduction}

Members of the genus Dunaliella (Chlorophyta, Chlorophyceae) are recognized as being the only eukaryotic photosynthetic organisms able to grow in an extremely wide range of salt concentrations. Dunaliella cells lack a rigid cell wall, instead, the cell is enclosed by a thin elastic plasma membrane. The alga is a model organism to study biochemical processes and represents one of the physiologically best studied unicellular green algae (González et al. 2001). Another important attribute is that these algae can accumulate carotenoids that are valuable substances for the health food and pharmaceutical industry. The generic type species D. salina (Dunal) Teodoresco is for example cultivated as a natural source of ß-carotene (Gómez and González 2004) or zeaxanthin (Jin and Melis 2003). To enhance the quantity of accumulated carotenoids, mutations are randomly introduced into cultures of Dunaliella. In this study three mutants of the wildtype strain D. salina CCAP 19/30 were investigated. These 
mutants were induced with the mutagenic chemical ethylmethane sulfonate (EMS, e.g. Jin et al. 2002). Although it is not possible to determine how many regions of the entire genome were mutated by this procedure, two mutants, VD 72 and VD 107, exhibit extreme production of carotenoids in comparison to their wildtype strain, whereas the mutant VD 6 accumulates lower amounts of this substance. A genetic comparison of the mutants and the wildtype is desirable to find out if the phenotypic differences can be correlated with differences on the molecular level. In addition, a genetic signature of the mutants can be helpful for their identification.

For this purpose the fingerprinting technique amplified fragment length polymorphism (AFLP, Vos et al. 1995) was chosen, since it was found to be suitable for distinguishing closely related organisms below the level of species and has been applied to a broad range of organisms (Mueller and LaReesa 1999, Savelkoul et al. 1999), including macro- and microalgae from marine and freshwater environments (e.g. Werner 2001, Mannschreck et al. 2002, John et al. 2004, Murphy and Schaffelke 2003, Erting et al. 2004). Using AFLP, different isolates of the same species (Chlorella vulgaris, Chapter 2) or UV light and radioisotope-induced mutants of the same isolate (Parachlorella kessleri, Chapter 3) could be distinguished.

A major advantage of the AFLP technique is that it generates a large number of polymorphic markers widely distributed across the genome of the studied species and that no prior knowledge of the genome is required. One limitation is that within the resulting AFLP banding patterns one cannot distinguish whether a fragment was generated by the DNA of the organism under study or by an additional, overlooked organism (contaminant). These contaminants can comprise bacteria, fungi and viruses as well as other algae. AFLP analyses of algal strains are thus reasonable only if axenic and unialgal cultures are investigated. Dunaliella is an example of a microalga for which purification is not easy to achieve. Microscopic inspection of D. salina CCAP 19/30 and its three mutants showed the presence of bacteria and possibly also fungi. Sequence analyses of an excised AFLP fragment of CCAP 19/30 revealed for example the presence of the marine proteobacterium Idiomarina loihiensis (S. Gäbler, pers. comm., accession number AE017340). However, it was likely that the same contaminants were present in all samples since the three mutant strains were isolated from the same wildtype culture. Therefore the AFLP technique was applied to them under the assumption that the same contaminant-specific fragments would be present in all samples and would not be evaluated as differences between a mutant and the wildtype. However, for the 
AFLP evaluation it had to be kept in mind, that polymorphic fragment positions could also represent genetic alterations of bacterial/fungal origin induced by the EMS treatment.

In addition, the axenic strain DCCBC 15 was included in the analyses. According to database records of the CCAP culture collection, this strain represents the same isolate as CCAP 19/30, but is free of contaminants. A comparison of AFLP banding patterns of the contaminated strain CCAP 19/30 and the axenic strain DCCBC 15 should therefore help to identify the amount of contaminant-specific fragments. DCCBC 15 and CCAP 19/30 were referred to as D. bardawil until 2001 when ITS rDNA analyses revealed this isolate to be more similar to D. salina (González et al. 2001). In the CCAP culture collection the strain CCAP 19/30 was therefore renamed D. salina, whereas the DCCBC culture collection still uses the old name D. bardawil (cf. Table 4.1). Based on ITS rDNA sequence analyses it was shown in this study that the database records were wrong and that these two strains are not duplicates. Detection of contaminant-specific fragments was thus, instead of comparing with patterns of DCCBC 15, achieved by comparing banding patterns generated from cultures with differing ratios of Dunaliella and contaminating bacteria and/or fungi. The differing ratios were achieved by following a washing procedure for each culture prior to DNA extraction. For further comparisons a second axenic strain of $D$. salina (DCCBC 2) was included in the analyses; this strain represents a different isolate than CCAP 19/30.

\subsection{Materials and Methods}

\subsubsection{Investigated strains and sample preparation}

Wildtype strain CCAP 19/30 and the three mutants VD 6, VD 72 and VD 107 were obtained from Prof. João Varela, Centro de Cincias do Mar (CCMar), University of Algarve (Faro, Portugal). Strains D. bardawil DCCBC 15 and D. salina DCCBC 2 were obtained from the DCCBC culture collection. DCCBC strains were axenic, while CCMar strains were contaminated with bacteria and perhaps also fungi. To minimize the effects of DNA from bacterial/fungal origins on the AFLP banding patterns, the cultures were washed 10 times. To test if this washing procedure really reduced the amount of fragments generated by DNA of the contaminating organisms, DNA of the wildtype was extracted in addition from the unwashed culture and the supernatant after the first centrifugation. Mutants were only investigated after the tenth wash step. Putative contaminant-specific fragments in the algae samples were identified as having a corresponding fragment in the banding pattern of the supernatant and the unwashed culture, and were excluded from the analyses. Washing procedure was performed as follows: all cultures were pelleted by centrifugation, the 
supernatant discarded and the pellet resuspended in axenic culture medium. This procedure was repeated 10 times. Each wash significantly reduced the amount of visible contaminants, but could not eliminate the contaminating organisms completely.

\subsubsection{DNA isolation and AFLP procedure}

DNA extraction and AFLP reactions were carried out as described for Chlorella vulgaris in Chapter 2, but instead of three replicates, only one DNA extraction and one AFLP reaction were performed per sample. The following eight primer combinations were used for selective amplification: EcoRI+AAG/MseI+C, EcoRI+AAG/MseI+G, EcoRI+AAG/MseI+T, EcoRI+AAG/MseI+A, EcoRI+ACG/MseI+C, EcoRI+ACG/MseI+G, EcoRI+ACG/MseI+T and EcoRI+ACG/MseI+A. Primers EcoRI+AAG and EcoRI+ACG were labelled with the fluorochromes 6-FAM and NED, respectively. Multiplex PCR with two EcoRI-primers gave the same results as PCR with only one EcoRI-primer. The fluorescently labelled, amplified fragments were separated on an ABI Prism 3100 (Applied Biosystems) capillary sequencer. Fragments up to 500 bp were sized with GeneScan-500 [LIZ] standard (Applied Biosystems).

\subsubsection{AFLP evaluation and phylogenetic analyses}

AFLP banding patterns were evaluated in two ways, i.e., automatically, to obtain a fast and objective cladistic analyses, and manually to control the automatic analysis and to identify putative contaminant-specific fragments. Automatic evaluation and phylogenetic analyses were conducted as described in Chapter 2.

\subsubsection{ITS rDNA sequence analyses}

The $18 \mathrm{~S}$ region of the rDNA of CCAP 19/30 was amplified, sequenced, evaluated and blasted as described in Chapter 2 for Chlorella vulgaris. Sequences of D. salina DCCBC 2 and D. bardawil DCCBC 15 (accession numbers ITS-1 and -2 AF313430 and AF313431) were obtained from Jürgen Polle, DCCBC culture collection. D. bardawil DCCBC 15 is the putative duplicate strain from CCAP 19/30. This strain is the authentic strain of D. bardawil Ben-Amotz et Avron isolated in Israel in 1976 and was renamed to D. salina at the CCAP culture collection (Table 4.1). DCCBC 2 represents a different isolate of $D$. salina, isolated on the western coastal side of South Korea. 


\subsection{Results}

Table 4.1 List of putative duplicate strains of the authentic isolate of $D$. bardawil Ben-Amotz et Avron that was isolated from a salt pond near Bardawil Lagoon (Israel) in 1976.

\begin{tabular}{lccc}
\hline \hline Strain number & Strain name & Origin & Axenic \\
\hline ATCC 30861 & D. bardawil & received from A. Ben-Amotz and M. Avron in 1976 & yes \\
\hline UTEX 2538 & D. bardawil & received from R. Adams in 1980 as ATCC 30861 & yes \\
\hline DCCBC 15 & D. bardawil & received from UTEX in 2002 & yes \\
\hline SAG 42.88 & D. salina & $\begin{array}{r}\text { received from M. Avron via Prof. Thomson in 1988 } \\
\text { as D. bardawil. It was renamed in 2001 }\end{array}$ & no \\
\hline CCAP 19/30 & D. salina & received from SAG in 1996 & no \\
\hline
\end{tabular}

\subsubsection{Automatic AFLP evaluation}

Automatic evaluation of AFLP banding patterns from the wildtype, the three mutant strains (all 10-fold washed) and both DCCBC strains was performed using the software GenScanner and resulted in a binary matrix of 258 characters. Phylogenetic analysis of this data set revealed that the wildtype strain and the mutants were clearly different from the DCCBC strains (Fig. 4.1). Relationships between the wildtype strain and the mutants revealed the three mutants to be more similar to each other. Within this group 27 (10.5\%) polymorphic fragment positions were found. Both DCCBC strains were more similar to each other than to the CCAP strain which was well supported in bootstrap analyses (Fig. 4.1).

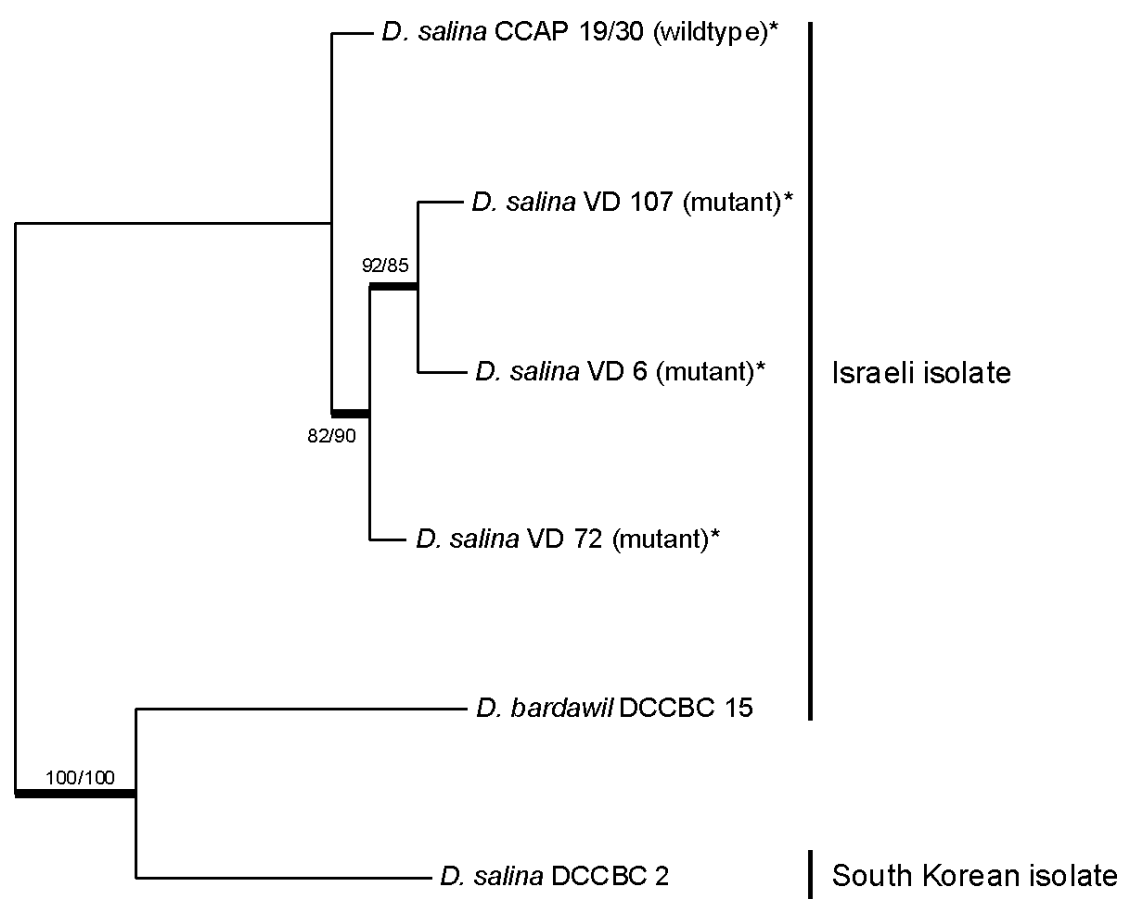

Fig. 4.1 Phylogeny of the studied Dunaliella strains based on AFLP automated evaluation. A mid-point rooted neighbor-joining $(\mathrm{NJ})$ tree is shown. Thick lines mark internal nodes that were resolved in both distance analyses (NJ) and maximum parsimony (MP). Asterisks denote samples washed 10-fold before AFLP analyses. Values above internal nodes are bootstrap values from 2000 replicates as calculated from NJ (left) and MP (right). Only values above 50\% are shown. 


\subsubsection{Manual AFLP evaluation}

Since it was known that the automatic evaluation may overestimate differences among strains with very similar banding patterns (Chapter 2), an additional comparison of all AFLP banding patterns was performed manually. Results of this manual comparison were in agreement with the findings of the automatic evaluation. The amount of differences among the wildtype and the mutants was, however, not found to be as high as revealed by automatic evaluation. Manual comparison resulted in 865 fragment positions and 22 were polymorphic between the wildtype CCAP 19/30 and the three mutants. These 22 polymorphic fragments represented $2.6 \%$ of all fragment positions, whereas in the automatic evaluation $10.5 \%$ of the positions obtained were polymorphic. In comparison to the automatic evaluation, almost three times as many fragments were manually evaluated because in the automatic evaluation only a subset of all fragments could be evaluated due to the use of a fragment intensity threshold above which a fragment was counted as present (cf. Chapter 2). Furthermore AFLP patterns of $D$. salina were found to be very reliable and of sufficient quality for evaluation. Therefore no further replicates were conducted as was usually done for AFLP investigations (cf. Chapter 2 and 3). Manual comparison of AFLP banding patterns revealed ca. 35\% differences between the CCAP strain and DCCBC 15, ca. 30\% differences between CCAP and DCCBC 2, and ca. 20\% differences between both DCCBC strains (not shown).

\subsubsection{Comparison of banding patterns from washed and unwashed cultures}

AFLP patterns from strain CCAP 19/30 prior to and after washing were considerably different (compare B and D in Fig. 4.2). Numerous fragments were present in patterns from the washed cultures, but were absent in the unwashed culture (asterisks in Fig. 4.2). All of the 22 polymorphic fragment positions found by manual evaluation had a corresponding fragment in the patterns generated from the unwashed sample or from the supernatant (e.g. arrows in Fig. 4.2). Some fragments were present in patterns of the supernatant, the unwashed and the 1-fold washed culture, but absent in the 10-fold washed cultures (arrowheads in Fig. 4.2). A number of fragments was present in all banding patterns (dots in Fig. 4.2). A last category of fragments was only absent in the patterns of the supernatant and therefore likely to be algaspecific (squares in Fig. 4.3). 


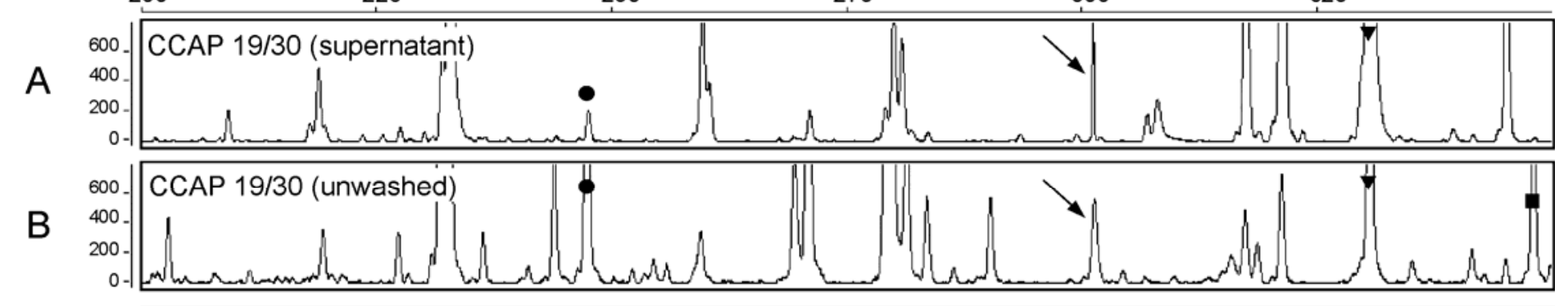

600 CCAP 19/30 (1-fold)

C

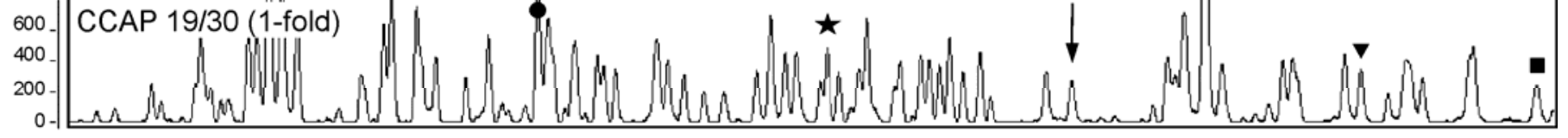

D

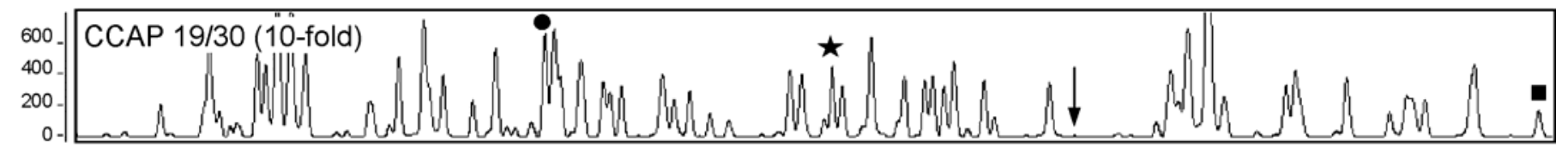

E

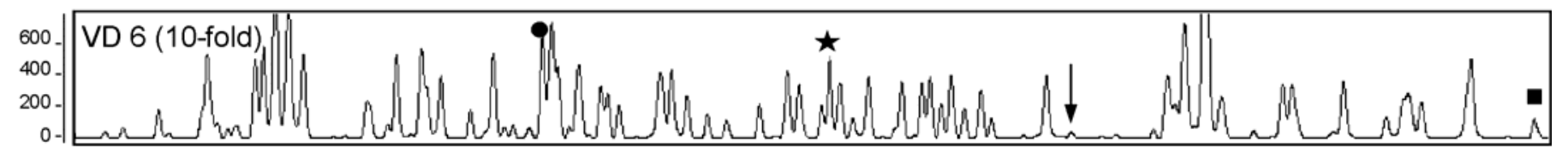

F $\quad \begin{array}{r}600 \\ 200 \\ 0\end{array}$
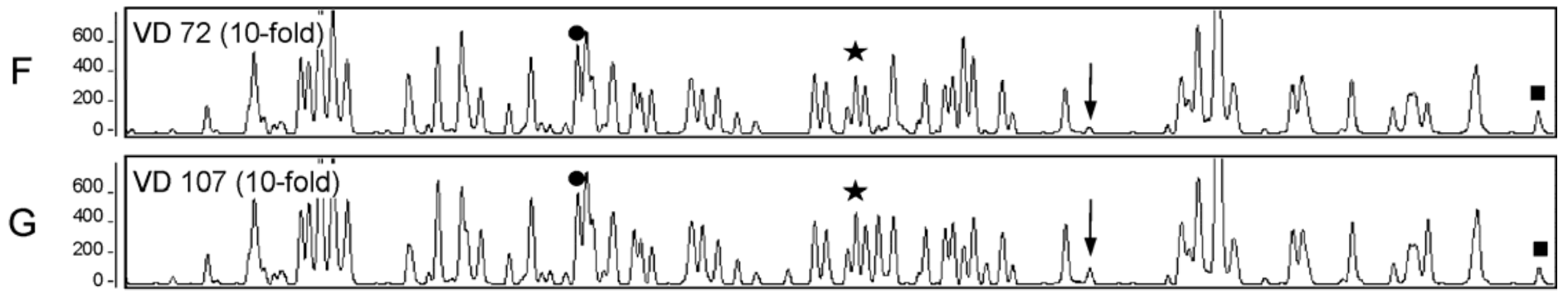

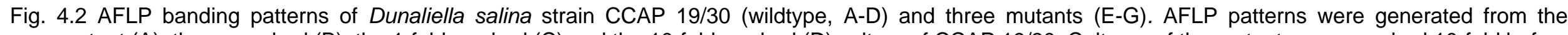

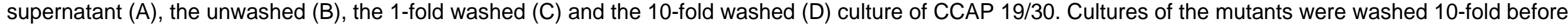

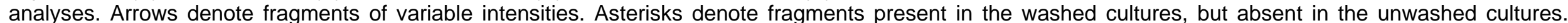

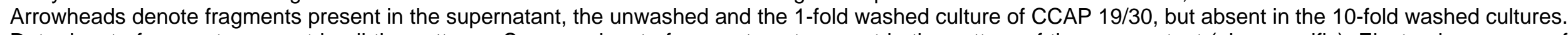

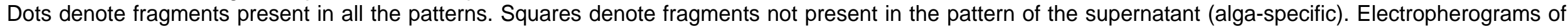
EcoRI+AAG/Msel+C. Vertical scales, relative fluorescent units; horizontal scales, size of fragment in nucleotides. 


\subsubsection{Correlation of ITS rDNA and AFLP analyses}

Results of the ITS rDNA sequence analysis of the wildtype and the two DCCBC strains differed from the findings of the AFLP evaluation. Specifically, identical sequences were obtained for CCAP 19/30 and DCCBC 2 which had six different positions in comparison to DCCBC 15, the putative duplicate strain of CCAP 19/30 (Fig. 4.3). Strain DCCBC 15 was therefore not the duplicate of CCAP 19/30 and could not be used for further AFLP evaluations to identify putative contaminant-specific fragments.

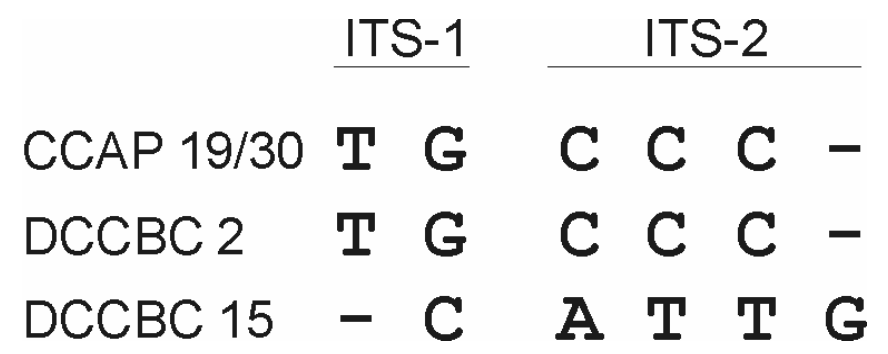

Fig. 4.3 Variable positions of ITS1-ITS2 sequences from Dunaliella salina CCAP 19/30 and DCCBC 2 and Dunaliella bardawil DCCDBC 15 (duplicate strain of CCAP 19/30). The six variable sequence positions are shown.

\subsection{Discussion}

\subsubsection{Influence of the contaminants on AFLP patterns}

Polymorphic fragments were contaminant-specific

All of the 22 polymorphic fragment positions found by manual evaluation had a corresponding fragment in the patterns generated from the unwashed sample or from the supernatant (cf. arrows in Fig. 4.2). Intensities (peak heights) of these polymorphic fragments were rather variable among the studied strains, with the highest intensities in the unwashed wildtype strain. It was thus likely that the detected polymorphisms were contaminant-specific and caused by variable amounts of contaminating DNA still present in the cultures rather than representing polymorphisms linked to mutations in $D$. salina. The variable concentrations of contaminating DNA could also represent genetic alterations of the bacteria or fungi that were induced by the mutagenesis procedure. However, both cases support the assumption that the polymorphic fragments were contaminant-specific and did not represent differences between the wildtype and the mutants.

Washing procedure reduced the amount of contaminant-specific fragments

The fact that the banding patterns of the supernatant and the unwashed culture were clearly different from the washed cultures made it evident that the patterns of the supernatant 
and the unwashed cultures mainly comprised contaminant-specific fragments. However, the efficiency of the washing steps in reducing the amount of contaminant-specific fragments in AFLP patterns was shown for fragments that were present in patterns of the supernatant, the unwashed and the 1-fold washed culture, but absent in the 10-fold washed cultures (arrowheads in Fig. 4.2).

\section{Contaminants led to atypical banding patterns for the 10-fold washed samples}

There is evidence that the patterns of the washed samples do not present typical patterns that would have been obtained of an axenic culture. Even for the 10-fold washed samples it can be expected that additional contaminant-specific fragments are present and that alga-specific fragments are missing due to a suppression in amplification. A combination of both cases led to atypical patterns for CCAP 19/30 and the mutants and are discussed below.

AFLP patterns from strain CCAP 19/30 prior to and after washing were considerably different (compare B and D in Fig. 4.2). Numerous fragments were present in patterns from the washed cultures, but were absent in the unwashed culture (asterisks in Fig. 4.2). This was probably due to a suppression of alga-specific fragments by DNA of contaminating organisms during DNA amplification. A suppression in amplification of AFLP fragments has not yet been reported, but is known for random amplified polymorphic DNA analyses (RAPD, Dyer and Leonard 2000). For AFLP, as for any other PCR-based method, such a suppression can be expected if different kinds of DNA are present and was also shown in this study for Chloromonas rosae (Chapter 5).

It is obvious that the AFLP technique is not selective for specific types of DNA and that the presence of additional organisms can lead to false banding patterns. Dyer \& Leonard (2000) demonstrated the influence on a fungal culture that was contaminated with undetected bacteria. This contaminant led to an extra genotype in their investigations and was only identified because this additional genotype had unique additional fragments compared to its most similar genotype. Missing fragments were not observed. The amount of putative contaminant-specific fragments found here for the D. salina strains was about $2.6 \%$ (22 of 865 fragments). This was therefore much lower than the amount of $14.6 \%$ obtained for a nonaxenic culture of Chloromonas rosae (Chapter 5.5.4) or 39 additional AFLP fragments in the study of Dyer \& Leonard (2000). In both latter cases, the contaminants were not easy to detect, e.g. not by visual inspection of the culture. This was also true for the 10-fold washed cultures of $D$. salina comprising the wildtype CCAP 19/30 and the three mutants, where the contaminants were only found after very careful microscopic investigation. It could therefore 
be expected that the influence of the contaminants on the banding patterns of the non-axenic D. salina samples might be higher than it was found here.

This assumption is supported by the presence of homologous fragments that were present in all patterns (supernatant, unwashed and washed cultures) and are denoted with dots in Fig. 4.2. For these fragments it was more likely that they were contaminant-specific than that they represented an alga-specific fragment of the same size. Comparison with the patterns obtained for DCCBC 2 supported the view that these fragments were more likely contaminant-specific, because most of them were not present in the patterns of the DCCBC 2 strain (not shown).

\subsubsection{Correlation of ITS rDNA and AFLP analyses}

Further evidence for the presence of unidentified contaminant-specific fragments in the patterns of the 10-fold washed cultures was provided by comparison of the AFLP and ITS rDNA sequence analysis that was performed for the wildtype strain CCAP 19/30 and the two DCCBC strains. DCCBC 15 represented the putative duplicate of CCAP 19/30 (Table 4.1, isolate from Israel) and DCCBC 2 was a different isolate of $D$. salina from South Korea. This comparison revealed ca. 35\% different AFLP fragments between CCAP 19/30 and DCCBC 15. The ITS sequences between both strains had six different positions. However, a lower amount of differences of ca. 30\% was found between CCAP 19/30 and DCCBC 2 that had identical ITS rDNA sequences. Furthermore, both DCCBC strains differed in only ca. 20\% of AFLP fragments even if they had six differing positions in ITS rDNA. It is assumed that a small amount of differences between ITS rDNA sequences is correlated with a much higher amount of differences between AFLP banding patterns. The AFLP banding patterns of CCAP 19/30 and DCCBC 2 with identical ITS rDNA sequences should therefore be more similar to each other than those of CCAP 19/30 and DCCBC 15, which had six different positions among the ITS rDNA region. Both DCCBC strains with six different positions in ITS rDNA had 20\% differences. CCAP and DCCBC 15 should therefore also have around 20\% differences because they likewise have six differing positions. Furthermore, CCAP and DCCBC 2 should have less than 20\% differences with their identical ITS rDNA sequences. The only explanation for this discrepancy is that even after a 10-fold washing procedure the amount of the contaminants was still high enough to influence the banding patterns of CCAP 19/30 and resulted in an atypical banding pattern for this strain and its three mutants. This atypical banding pattern led to the differences and especially high differences in comparison to DCCBC 2. 
In comparison to differences found within AFLP banding patterns of different isolates of Chlorella vulgaris (Chapter 2) that had identical ITS rDNA sequences, the differences found in the present study were clearly lower. The isolates of $C$. vulgaris showed between 20 and 40\% differences in AFLP analyses (data not shown). The amount of differences found for Dunaliella between both DCCBC strains was much lower, with $20 \%$ in AFLP but six different positions in ITS rDNA. It is possible that this is attributable to the fact that D. salina and $C$. vulgaris are quite different organisms and therefore show also differences on the genomic level. However, a correlation between ITS rDNA and AFLP as found for C. vulgaris was found for a number of organisms including algae (Pelser et al. 2003, Erting et al. 2004, John et al. 2004) and therefore might also be expected for Dunaliella.

\subsubsection{Identity of duplicate strains}

ITS rDNA analyses revealed that CCAP 19/30 and DCCBC 15 had six different positions among their ITS rDNA regions. This level of differences is sufficiently high that any possibility of the cultures originating from the same isolate can be ruled out. This can be explained by the frequent exchange of strains between different culture collections and that these exchanges were only incompletely recorded in the collection's databases (Table 4.1). For example, the DCCBC collection obtained the strain DCCBC 15, which should present the duplicate of CCAP 19/30, from the UTEX culture collection as ATCC 30861. According to database entries of the different collections, this isolate was isolated in Israel in 1976 and was sent to the ATCC culture collection (Table 4.1). The SAG culture collection obtained a strain of this isolate in 1988 from M. Avron via Prof. Thomson. The CCAP culture collection reinstated it from the SAG culture collection in 1996 and later sent it to João Varela at CCMar, who induced the mutants VD 6, VD 72 and VD 107. Therefore it is possible that, due to mislabeling, CCAP 19/30 and DCCBC 15 do not represent duplicate strains. This is known for a couple of strains and was quite often found in culture collections, e.g., Chlorella vulgaris (Chapter 2), Parachlorella kessleri (Chapter 3), Chlamydomonas (T. Pröschold, pers. comm.) or cyanobacteria such as Anabaena variabilis (Sauer 2004). A different origin of the SAG and the CCAP strain is also supported by the assumption of João Varela that D. salina CCAP 19/30 depends on bacteria and cannot survive purification (J. Varela, pers. comm.). This finding is in contrast to the axenity of the putative duplicate strains and support the hypothesis of their different origins. 


\subsubsection{Comparison of the wildtype and the mutants}

All 22 polymorphic fragments were found to be more likely contaminant-specific than to present a mutation between the wildtype and the mutants. For their identification as contaminant-specific, it was necessary that the same type of contaminant was present in all samples and that banding patterns generated from cultures with differing ratios of algae and contaminants were compared. Using this procedure it was shown that AFLP is also suitable to investigate non-axenic cultures. This is an important alternative for strains such as $D$. salina that are not as easy to maintain in an axenic status.

No differences could therefore be found between the wildtype and the EMS-induced mutants among the 865 manually evaluated AFLP fragment positions. The mutations present in D. salina are smaller than those derived from UV light- or radioisotope-induced mutants of Parachlorella kessleri, where up to eight of 285 fragment positions were found to differ in comparison to the wildtype (Chapter 3). This result could be expected because it is known that UV light produces a greater range of substitutions than EMS (Lawrence 2002). UV lightinduced mutations mostly occur in runs of pyrimidines, particularly T-T pairs, and include both transitions and transversions. UV also induces a siginificant frequency of frameshift mutations. The widely used agent EMS is alkylating and highly specific in its activity; it almost exclusively produces transitions at G-C sites to A-T (Lawrence 2002). The restriction sites of the enzymes MseI and EcoRI used here for AFLP analyses are T'TAA and G'AATTC, respectively, and are thus optimal to detect mutations induced by EMS. The rate of induced mutations was obviously below the discrimination level of the AFLP technique with the enzyme- and primer combinations used here. Another example in which the extent of mutations present in $D$. salina was estimated is given by investigations between different mating types of Chlamydomonas reinhardtii. Sixteen polymorphic fragments were obtained with seven EcoRI/MseI primer combinations, eight of them linked to the mating type locus (Werner et al. 2001). In D. salina, eight EcoRI/MseI primer combinations were used which might indicate that the investigated mutants show less variation than the mating types of $C$. reinhardtii. On the other hand one must keep in mind that with AFLP any given individual mutation may have only been found by chance, and that further analyses could be conducted using additional enzyme- and/or primer combinations to search for mutations in these $D$. salina strains. An additional aspect is that these two examples are members of the Trebouxiophyceae whereas $D$. salina is a member of the Chlorophyceae; it is not known to what extent members of these groups are comparable. Notwithstanding, it is concluded that mutations in $D$. salina are smaller than in the other examples. 


\subsubsection{Comparison of automatic and manual evaluation}

Phylogenetic analysis of the automatically evaluated AFLP banding patterns was found to be an adequate method to distinguish between the 10 -fold washed samples of $D$. salina and both DCCBC strains. In all, 10.5\% differences were found by automatic evaluation within the 10 -fold washed samples, whereas manual evaluation revealed that only $2.6 \%$ of these differences were true differences. This overestimation of differences produced by automatic evaluation was also obtained for Chlorella vulgaris and is due to varying fragment intensities between samples. Varying fragment intensities are problematic for the softwareassisted evaluation (cf. Chapter 2, Fig. 2.4). For Chlorella vulgaris it was demonstrated for the first time and here for the second time that automatic evaluation is accurate and reliable for a rough and objective analysis that can be performed within minutes. These are clear advantages over manual evaluation, which is time-consuming and subjective (cf. Chapter 2.5.8). A manual comparison only has to be performed for patterns that were found to be very similar (clustered together) during automatic evaluation. 


\section{Amplified fragment length polymorphism (AFLP) in genetic stability tests of cryopreserved microalgae in combination with reproducibility testing of AFLP banding patterns}

\subsection{Abstract}

Cryopreservation has become the method of choice for the long-term preservation of microalgae as it should theoretically guarantee genetically stable cultures over several decades. However, the genetic integrity of microalgae after cryopreservation has not been investigated at the molecular level to date. In this study 29 strains of microalgae were examined using the fingerprinting method amplified fragment length polymorphism. The strains investigated were mostly Chlorophyta with diverse morphological and physiological properties, and included strains known to survive cryopreservation with high viability levels, as well as cryo-recalcitrant strains. Initially, the reproducibility of the AFLP technique was tested for all strains by comparing three independently replicated banding patterns per strain. This resulted in a mean error rate of $0.9 \%$ non-reproducible fragments from the technique. All strains were cryopreserved using a twostep cooling procedure. After cryopreservation, mean differences of $1.3 \%$ were observed in 15 strains, independent of the determined error rate. Reasons for the differences observed may potentially be due to changes in DNA methylation, cryo-selection of subpopulations, or cryo-induced mutations. Contaminants could be excluded as sources of differing fragments. For seven strains post-cryo differences observed were in the same range as found between pigment mutants of Parachlorella kessleri and their wildtype.

\subsection{Introduction}

In recent years, cryopreservation has become the method of choice for long-term preservation of eukaryotic microalgae and cyanobacteria (Benson 2004, Brand and Diller 2004). However, cryogenic storage of algal cultures has been performed since the 1960s (Holm-Hansen 1963) and a number of 'robust' protocols are available for a range of algal taxa (Morris 1978, Bodas et al. 1995, Day 1998). For review on the extensive literature see Taylor \& Fletcher (1999), or for overview of techniques Day and Brand (2005). One of the main advantages of cryopreservation compared to other techniques (e. g. traditional serial transfer) is that it is assumed to prevent genetic changes due to environmental selective pressures and/or genetic drift, thus ensuring genetic stability of preserved organisms (Day and Brand 2005). At $-196^{\circ} \mathrm{C}$ cell survival is independent of the period of storage and biological systems 
are genetically stable. However, the cryopreservation process may itself induce enormous stresses on the organisms. These stresses include a number of factors that can cause cryoinjury, e.g. intracellular ice formation, cryoprotectant toxicity, osmotic shock, freeze-fracture events and gross mechanical damage (Fleck 1998). The effects of cryo-injury upon the genome of an organism are mostly unknown (Harding 2004). Although it is, for example, known that they can lead to the metabolic response of production of highly toxic hydroxyl radicals that can damage the DNA (Fleck et al. 2000). This DNA damage mediated by free radicals has important consequences for genetic stability of cryopreserved materials (Benson and Bremner 2004). All these critical factors upon the genome make the assessment of genetic integrity after cryogenic storage desirable and can be investigated on the phenotypic, histological, cytological, biochemical and molecular levels (Harding 2004). On the molecular level, analyses of genomic DNA sequences before and after cryopreservation have been performed for higher plants (e.g., potatoes, strawberry, apple) or using hybridization and a range of PCR techniques; several of these investigations report evidence of stability after cryopreservation (for review see Harding 2004). However, the genetic integrity of cryopreserved microalgae has not been investigated to date.

The amplified fragment length polymorphism fingerprinting technique (AFLP, Vos et al. 1995) was chosen to investigate 29 strains of microalgae before and after cryopreservation. This technique has already been used in several studies on higher plants to investigate genetic stability after cryopreservation, without finding any differences (Hao et al. 2001, Turner 2001, Wilkinson et al. 2003, Liu et al. 2004). In other studies minor differences of less than $0.3 \%$ of all investigated AFLP fragments were observed, which were assumed to be likely due to cryogenic induced changes in DNA methylation (Hao et al. 2002, Helliot et al. 2002).

AFLP is known to be a reliable and reproducible fingerprinting technique to resolve differences between isolates of the same species and has been used for a broad range of taxa including bacteria, animals and plants (Mueller and LaReesa 1999, Savelkoul et al. 1999). It has been shown that this technique was also appropriate to distinguish different isolates of the same species of the green microalgae Chlorella vulgaris with good reproducibility (Chapter 2). However, this technique has only been employed with three microalgae: Chlamydomonas reinhardtii (Werner et al. 2001), Asterionella formosa (De Bruin et al. 2004) and Alexandrium tamarense (John et al. 2004).

The present paper is concerned with two separate but interconnected research questions. First, the reproducibility of the AFLP technique was examined; and secondly, the genetic stability of these microalgae after cryopreservation was investigated. The strains 
studied comprised mainly members of the Chlorophyta, which is the best studied group of microalgae in the context of cryopreservation (e.g. Morris 1978, Beaty and Parker 1992). Included were taxa known to survive cryopreservation with high viability levels (e.g. Chlorella, Nostoc) as well as cryo-recalcitrant organisms (e.g. Pseudendocloniopsis, Chlamydocapsa). In addition, multiple species from the same genus (Chlamydomonas and Trebouxia, see Fig. 1.3d for T. asymmetrica) as well as strains representing the same isolate but maintained separately in different culture collections (Coccomyxa arvernensis, Fig. 1.3g) were studied. Furthermore, strains with strongly differing morphological and physiological properties were included, as for instance unicellular Chlorella vulgaris (Fig. 1.3a) with rigid cell walls, Uronema belkae which has the ability to form filaments (Fig. 1.3h), gelatinous cultures of Coccomyxa arvernensis, motile strains such as Chlamydomonas or non-motile and colony-forming strains such as Coelastrum morum (Fig. 1.3e) or of complex and manifold cell morphology such as Cosmarium cucumis (Fig. 1.3f). Also included were two strains known to be difficult to cryopreserve, namely Chlamydomonas reinhardtii (Crutchfield et al. 1999, Brand and Diller 2004) and Euglena gracilis (Morris and Canning 1978, Day et al. 1997, Day 1998, Day et al. 2000). Members of the genus Euglena have contractile vacuoles and this is one reason why cryopreservation of strains of this genus is problematic. AFLP analyses were performed on all cultures directly after thawing and subsequently on the regrown cultures. In an extended cryopreservation experiment, six of the 29 strains were cryopreserved for three successive cycles and analysed with AFLP after each procedure to investigate whether any kind of cryo-selection occurred and the influence of this continuous stress on the organisms.

Although AFLP has in general been found to be a reliable and reproducible technique, it is known from previous reports that its reproducibility is dependent on the quality of the DNA in investigation (Savelkoul et al. 1999, Donaldson et al. 2000). The influence of DNA quality was assessed by investigation of strains with strongly differing morphology that might influence the success of DNA extraction. In addition, a strain for which DNA extraction is known to be difficult, Amphikrikos sp. was included (Hepperle et al. 2000). Furthermore, the influence of bacterial contaminants on the AFLP patterns was determined by the inclusion of a strain of Chloromonas rosae that was not axenic after cryopreservation.

The aim of the AFLP analyses before and after cryopreservation was to find putative cryoinduced mutations. Therefore two pigment mutants and their wildtype strain were investigated, to find out whether AFLP is capable of detecting these mutations, and if so, how great their influence was on the banding patterns. The mutations were induced by UV light or 
radioisotope treatment of a wildtype strain of Parachlorella kessleri and exhibit clear differences in their phenotypes, e.g. colour and carotenoid biosynthesis (cf. Chapter 2).

\subsection{Material and Methods}

\subsubsection{Investigated strains and culture conditions}

The strains investigated (Table 5.1) were obtained from Culture Collection of Algae at Göttingen University (SAG, Göttingen, Germany), the Pasteur Culture Collection of Cyanobacterial Strains (PCC, Paris, France) or the Culture Collection of Algae and Protozoa (CCAP, Dunbeg, UK), and were cultured and cryopreserved in their appropriate medium. Media recipes are available from the SAG culture catalogue (http://www.epsag.unigoettingen.de). Strains were maintained in test tubes in liquid, or on agar slopes, under identical culture regimes: $20^{\circ} \mathrm{C}, 50 \mu \mathrm{mol} \mathrm{m} \mathrm{s}^{-2}$ (14/10h light/dark).

\subsubsection{Test for contaminants}

Cultures were checked for axenity before and after cryopreservation on agarized medium containing proteose peptone and saccharose (Trebouxia Organic Medium, Ahmadjian 1967). Parallel tubes were incubated for three days in darkness at $37^{\circ} \mathrm{C}$ and in light at $20^{\circ} \mathrm{C}$, and were checked for contamination both macroscopically and under an inverted microscope.

\subsubsection{Genetic analyses}

DNA extraction and AFLP. DNA extraction, restriction, ligation, preselective and selective amplification were carried out as described in Chapter 2 for Chlorella vulgaris, using $\quad E c o R I+A / M s e I+C, \quad E c o R I+G / M s e I+C$ and $E c o R I+C / M s e I+C$ for selective amplification. For Chlamydomonas reinhardtii primer combinations EcoRI+A/MseI+TG, EcoRI+C/MseI+TG and EcoRI+G/MseI+TG and for Cosmarium cucumis, Euglena gracilis and Phaeodactylum tricornutum EcoRI+AAG/MseI+C and EcoRI+ACT/MseI+C were used. For Macrochloris radiosa, Uronema belkae, Trebouxia sp. and Chloromonas rosae primer combinations EcoRI+A/MseI+CG, EcoRI+C/MseI+CG and EcoRI+G/MseI+CG were used. Fragment sizing. The fluorescently labelled amplified fragments were analysed by capillary electrophoresis using an ABI Prism 3100 (Applied Biosystems Foster City, CA, USA). The inclusion of the internal size standard GeneScan-500 [LIZ] (Applied Biosystems) enabled the 
ABI GeneScan software (Applied Biosystems) to assign the appropriate size to all fragments in the range of 35 to $500 \mathrm{bp}$.

\subsubsection{Reproducibility tests and evaluation}

AFLP analyses were performed a total of five times per sample, three times before and twice after cryopreservation. To assess the level of reproducibility, genomic DNA was extracted twice per sample before cryopreservation (pre-cryo) and the complete AFLP procedure (beginning with the restriction/ligation reaction) was performed twice for the first DNA extraction and once for the second. The number of non-reproducible fragments within these three pre-cryo replications was counted by comparing the three banding patterns obtained displayed in the software GenScan (Applied Biosystems). A fragment was counted as non-reproducible if it was present only in one or two of the three replicates. The amount of non-reproducible fragments was calculated as a percentage of all the fragment positions and provided the error rate of the technique. After cryopreservation (post-cryo), the DNA of the culture was investigated directly after thawing, dilution with fresh medium and $12 \mathrm{~h}$ recovery in darkness. In addition, the DNA of the regrown culture (two to three weeks after thawing) was also studied. Banding patterns were manually compared with the three pre-cryo patterns and additional or missing fragments were counted and expressed as a percentage. An additional or missing fragment was only counted if its position was reproducible, i.e. a postcryo fragment was counted as additional if no homologous fragment was present in any of the three pre-cryo replications. If a fragment was present only in one or two of the pre-cryo patterns, then it was a considered to be a non-reproducible fragment position and not counted after cryopreservation. Using this approach, the error rate of the technique that was calculated in reproducibility tests before cryopreservation was subtracted from all differences found in the post-cryo patterns. The amount of differing fragments observed post-cryo was expressed as a percentage of all reproducible fragment positions. 


\subsubsection{Cryopreservation protocol}

For outline of the cryopreservation protocol see Fig. 1.2b. Samples were adjusted to a

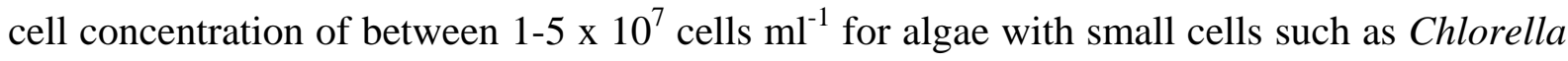
or Chlamydomonas. For algae with larger cells such as Euglena or Cosmarium equivalent cell density was used. The use of centrifugation to concentrate the amount of cells per ml was avoided and the use of a slightly different cell density was preferred over the additional stress of centrifugation on the organisms.

All strains were cryopreserved using the same two-step controlled rate cooling protocol. The algae were pre-treated with cryoprotectant (see below) in cryovials $(1.5 \mathrm{ml}$, Corning Incorporated, Europe) for $10 \mathrm{~min}$ on ice prior to transfer to a controlled-rate cooler (Planer, UK), precooled to $4^{\circ} \mathrm{C}$. Cultures were cooled with a cooling rate of $-1^{\circ} \mathrm{C} \min ^{-1}$ to an intermediate holding temperature of $-35^{\circ} \mathrm{C}$ and held at that temperature for $40 \mathrm{~min}$. An additional cooling step of $-1^{\circ} \mathrm{C} \min ^{-1}$ to $-45^{\circ} \mathrm{C}$ was used to guarantee that the samples held a temperature below $-35^{\circ} \mathrm{C}$ during the transfer from the controlled rate cooler to the cryostore vessel where the samples were directly placed into the vapour phase of liquid nitrogen ($176^{\circ} \mathrm{C}$ ). To minimize the risk of contaminants, plunging directly in liquid nitrogen was avoided. Samples were stored at least $24 \mathrm{~h}$ in the cryostore vessel before thawing. Samples were thawed by direct immersion of the cryovials in a water bath of $45^{\circ} \mathrm{C}$, until the ice had melted (2-3 min). To circumvent the additional stress of a centrifugation step and to prevent toxicity of the cryoprotectant, the samples were diluted 20-fold with fresh medium reducing the concentration to $0.25 \%$ cryoprotectant in the post-cryo culture. This concentration has previously been found not to cause any inhibition of the algal growth (Fleck, 1998). Diluted samples were transferred in parallel also onto agarized medium. Cultures were kept in darkness for $12 \mathrm{~h}$ and then under the same culture regimes as before the cryopreservation procedure $\left(20^{\circ} \mathrm{C}, 50 \mu \mathrm{mol} \mathrm{m} \mathrm{s}^{-2}, 14 / 10 \mathrm{~h}\right.$ light/dark).

\subsubsection{Choice of cryoprotectant}

With exception of two strains, 5\% [v/v] dimethylsulfoxide (DMSO) was used for all strains, because it is the most frequently applied and successful cryoprotectant in microalgae (Day and McLellan 1995, Taylor and Fletcher 1999). However, for Chlamydomonas reinhardtii and Euglena gracilis, methanol (5 and 10\%[v/v], respectively) was used following the methods of Day et al. (2000) and Brand and Diller (2004). 


\subsubsection{Post-thaw viability}

Post-thaw viabilities of the regrown cultures in liquid and agarized medium were determined by visual inspection fourteen days after thawing. Culture growth behaviour was assigned to five categories ranging from no regrowth $(-)$ to very good regrowth $(++++)$, with intermediates for very poor $(+)$, poor $(++)$ and good regrowth $(+++)$.

\subsubsection{Three cycles of cryopreservation}

In an extended experiment, six strains were cryopreserved for three successive cycles. These strains are denoted with an asterisk in Table 5.1. As soon as the culture was regrown after cryopreservation it was cryopreserved for a second or third time. An identical cryopreservation procedure was used for each cryopreservation cycle. In this experiment the AFLP analyses were performed only for the regrown cultures and not on the cultures directly after thawing.

\subsection{Results}

\subsubsection{Choice of AFLP primer combinations}

The AFLP technique was successfully applied to all 29 strains in this study. An identical AFLP protocol could be used for all strains. However, for some strains the number of selective nucleotides (two, three or four) in the selective amplification was adapted in order to increase or decrease the amount of amplified fragments. All samples were investigated using the primer combinations $E c o R I+A / M s e I+C, E c o R I+C / M s e I+C$ and $E c o R I+G / M s e I+C$, which had proved to be suitable for Chlorella vulgaris, Scenedesmus obliquus or Coccomyxa in preliminary tests. For logistical reasons, the AFLP analyses were performed on groups of five to ten strains at a time. Only for samples of the last groups of strains additional primer combinations were used with three or four selective nucleotides (EcoRI+A/MseI+CG, $\mathrm{EcoRI}+\mathrm{C} / \mathrm{Mse} \mathrm{I}+\mathrm{CG}$ and EcoRI+G/MseI+CG or EcoRI+AAG/MseI+C and EcoRI+ACT/MseI+C) (Table 5.1). These were randomly chosen as primers frequently used in AFLP studies of higher plants. For six strains these primer combinations led to an improved number of fragments in comparison to selective amplification with two selective nucleotides. The number of fragments was improved with an increase in clearly separated fragments evenly distributed from 100 to 500 base pairs. For Chlamydomonas reinhardtii the primer combinations $E c o \mathrm{RI}+\mathrm{A} / M s e \mathrm{I}+\mathrm{TG}, E c o \mathrm{RI}+\mathrm{C} / M s e \mathrm{I}+\mathrm{TG}$ and $E c o \mathrm{RI}+\mathrm{G} / \mathrm{Mse} \mathrm{I}+\mathrm{TG}$ were tested and evaluated, because these had been previously found to be suitable for this species by 
Werner et al. (2001). With these primer combinations, banding patterns representing well separated fragments were obtained and between 113 and 371 fragments were evaluated per strain (Table 5.1).

\subsubsection{Reproducibility of AFLP banding patterns}

Reproducibility of AFLP banding patterns for each of the 29 strains was assessed by comparing three replicated patterns per strain before cryopreservation. These three replications comprised two AFLP patterns that were independently obtained using a first DNA extraction of the culture, and the third pattern was obtained using a second DNA extraction of the same culture. In this way the influence of different DNA extractions on the patterns could be estimated, as well as the influence of two independent AFLP reactions on the same DNA extraction. The three banding patterns were manually compared and the nonreproducible fragments counted and expressed as a percentage of all fragment positions (number of reproducible plus non-reproducible fragments). A fragment was counted as nonreproducible if it was not present in one or two of the three replicates. Reproducibility within the three pre-cryo replications was on average $99.1 \%$ and ranged from 0 to $8.5 \%$ of nonreproducible fragments (Table 5.1). Non-reproducible fragments are denoted with arrowheads in Fig. 5.1 for Macrochloris radiosa, in Fig. 5.2 for Chloromonas rosae and in Fig. 5.3 for Nostoc sp. Parts of the banding patterns of these three strains are shown here as examples of all strains showing typical characters within these banding patterns. The majority of the nonreproducible fragments were found between banding patterns of the two independent DNA extractions, but some were also observed between replicates of the same DNA extraction. In most cases the independently replicated banding patterns of the same DNA extraction were more similar to each other than to the banding pattern of the different DNA extraction. This is shown for Macrochloris radiosa in Fig. 5.1. Here, banding patterns of the first DNA extraction (electropherogram A and B) are more similar to each other in fragment intensity and shape than to the pattern of the second DNA extraction (electropherogram C). However, this was not the situation for all samples. Patterns obtained for Chloromonas rosae (Fig. 5.2) or Nostoc sp. (Fig. 5.3) had the same amount of dissimilarities and similarities between all three pre-cryo replicates. It was noticeable that, compared to the peak intensities obtained for the whole banding pattern, some of the non-reproducible fragments had comparatively low peak intensities, close to background levels. Such fragments represented an average of $0.3 \%$ of all non-reproducible fragments and included both non-reproducible fragments shown for 
Macrochloris radiosa in Fig. 5.1, one of the four non-reproducible fragments of Chloromonas rosae shown in Fig. 5.2. However, none of the non-reproducible fragments of Nostoc sp. could be assigned to this fragment type (Fig. 5.3). The high levels of non-reproducible fragments of $8.5 \%$ for Amphikrikos sp. was considered to be a result of non-reliable AFLP reactions due to poor DNA quality. Accordingly, data from Amphikrikos sp. were excluded from the average reproducibility calculations.

\subsubsection{AFLP differences between mutant and wildtype}

Differences between the wildtype strain of Parachlorella kessleri and the two corresponding mutants were found to represent $2.8 \%$ for the UV light-induced mutant SAG 11.80 and $1.8 \%$ for the radioisotope-induced mutant SAG 211-11h/9. These differences were only counted for reproducible fragment positions by comparing the three pre-cryo banding patterns per strain. An example of banding patterns of the wildtype and the UV light mutant SAG 14.80, which was not investigated in this study, are shown in Chapter 3 where additional information on these pigment mutants are given.

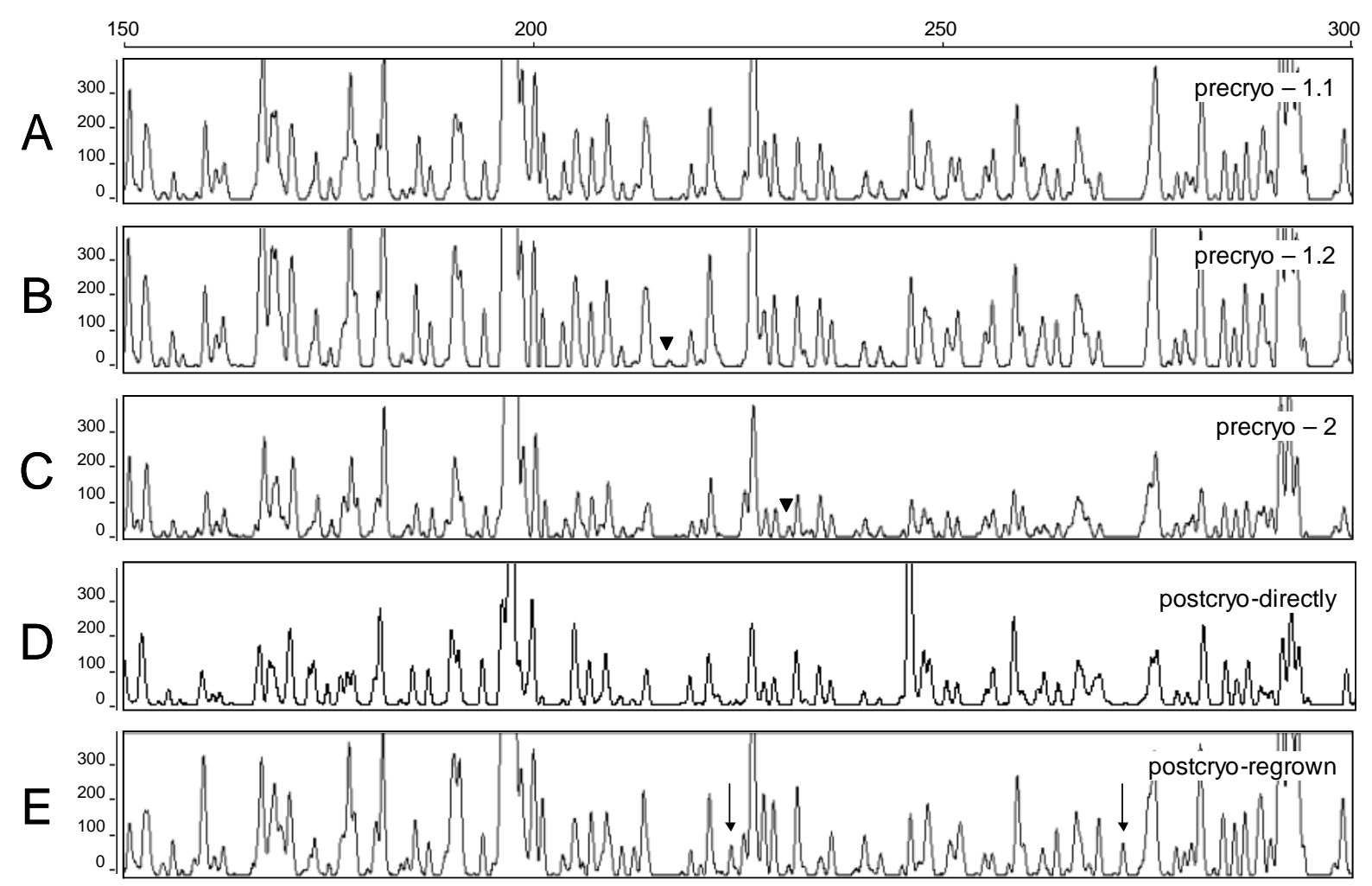

Fig 5.1 AFLP patterns of Macrochloris radiosa SAG 213-2a before (A-C) and after cryopreservation (D and $E$ ). Arrowheads denote non-reproducible fragments within the three pre-cryo banding patterns (A$C)$. Arrows denote additional fragments in the post-cryo banding pattern (E) of the regrown culture. Electropherograms from selective amplification with primer combination, EcoRI+C / Msel+TG. Vertical scales, relative fluorescent units; horizontal scales, size of fragment in nucleotides. 
Table 5.1 Cryopreserved strains, post-thaw viability levels and AFLP results: amount of nonreproducible (non-repr.) fragments before cryopreservation, amount of AFLP differences (diff.) after cryopreservation from a DNA extraction directly after thawing or from the regrown culture. Taxa represent members of the Trebouxiophyceae $(T)$, Chlorophyceae $(C h)$, Zygnematophyceae $(Z)$, Euglenophyceae (E), Bacillariophyceae (B) and Cyanobacteria (Cy). Four groups of strains are distinguished according to post-cryo differences between the regrown cultures.

\begin{tabular}{|c|c|c|c|c|c|c|c|}
\hline Strain number & Genus and species & Taxon & $\begin{array}{l}\text { Post-thaw } \\
\text { viability }^{\text {a }}\end{array}$ & $\begin{array}{c}\text { AFLP } \\
\text { fragments }\end{array}$ & $\begin{array}{l}\text { Pre-cryo } \\
\text { non-repr. }\end{array}$ & $\begin{array}{l}\text { Post-cryo } \\
\text { diff.direct }\end{array}$ & $\begin{array}{l}\text { Post-cryo } \\
\text { diff.regrown }\end{array}$ \\
\hline SAG 75.81 & Chlamydomonas meslinii & $\mathrm{Ch}$ & +++ & 254 & 0.8 & 0.0 & 0.0 \\
\hline SAG $11-32 b$ & Chlamydomonas reinhardtii* & $\mathrm{Ch}$ & +++ & $234^{\mathrm{C}}$ & 0.9 & 0.0 & 0.0 \\
\hline SAG 24.85 & Chlamydopodium. bulgar. & Ch & ++++ & 143 & 0.7 & 0.0 & 0.0 \\
\hline SAG 11.80 & Parachlorella kessleri & $\mathrm{T}$ & +++ & 277 & 1.1 & 0.0 & 0.0 \\
\hline SAG 211-11h & Parachlorella kessleri & $\mathrm{T}$ & ++++ & 283 & 0.0 & 0.0 & 0.0 \\
\hline SAG 211-11h/9 & Parachlorella kessleri & $\mathrm{T}$ & ++++ & 282 & 0.0 & 0.0 & 0.0 \\
\hline SAG 211-11b & Chlorella vulgaris* & $\mathrm{T}$ & ++++ & 132 & 3.0 & 0.0 & 0.0 \\
\hline SAG 612-10 & Cosmarium cucumis* & $\mathrm{Z}$ & +++ & $133^{c}$ & 1.5 & 0.0 & 0.0 \\
\hline SAG 1224-5/25 & Euglena gracilis* & $\mathrm{E}$ & +++ & $312^{\mathrm{C}}$ & 0.0 & 0.0 & 0.0 \\
\hline SAG 1090-1a & Phaeodactylum tricornutum* & B & ++++ & 167 & 0.6 & 0.0 & 0.0 \\
\hline$\overline{\text { SAG 216-1 }}$ & Coccomyxa arvernensis & $\mathrm{T}$ & ++ & 211 & 0.5 & 0.0 & 0.5 \\
\hline SAG 463-2 & Leptosira terrestris & $\mathrm{T}$ & ++ & 348 & 0.6 & 0.3 & 0.6 \\
\hline SAG 48.88 & Trebouxia asymmetrica & $\mathrm{T}$ & + & 329 & 0.0 & 0.3 & 0.6 \\
\hline SAG 249-1 & Muriella aurantiaca & $\mathrm{Ch}$ & ++ & 113 & 3.6 & 0.0 & 0.9 \\
\hline SAG 30.95 & Axilosphaera vegetata & $\mathrm{Ch}$ & ++ & 185 & 0.5 & 0.5 & 1.1 \\
\hline SAG 15.98 & Desmodesmus schnepfii & $\mathrm{Ch}$ & +++ & 274 & 0.7 & 0.0 & 1.1 \\
\hline SAG 213-2a & Macrochloris radiosa & Ch & +++ & $371^{\mathrm{C}}$ & 0.0 & 0.0 & 1.1 \\
\hline PCC 7120 & Nostoc sp.* & $\mathrm{Cy}$ & ++++ & 188 & 1.6 & 0.0 & 1.6 \\
\hline Hg 1999-5 & Coelastrum morum & Ch & +++ & 285 & 0.4 & 0.4 & 1.8 \\
\hline SAG 34.86 & Uronema belkae & $\mathrm{Ch}$ & ++++ & $193^{c}$ & 0.5 & 0.0 & 2.6 \\
\hline CCAP 216/1 & Coccomyxa arvernensis & $\mathrm{T}$ & ++ & 211 & 1.4 & 0.0 & 2.8 \\
\hline SAG 465-1 & Pseudendocloniopsis botry. & $\mathrm{Ch}$ & + & 339 & 0.3 & 0.3 & 3.2 \\
\hline SAG 11-61b & Chlamydomonas moewusii & $\mathrm{Ch}$ & ++ & 307 & 0.3 & 0.3 & 3.9 \\
\hline 98.006 C3 & Trebouxia sp. & $\mathrm{T}$ & +++ & $248^{\mathrm{C}}$ & 2.8 & 0.4 & 4.0 \\
\hline SAG 276-1 & Scenedesmus obliquus & Ch & ++++ & 315 & 0.6 & 0.0 & 6.3 \\
\hline Hg 1995-26 & Amphikrikos sp. & Ch & +++ & 129 & 8.5 & $1.6^{\mathrm{e}}$ & $10.1^{\mathrm{e}}$ \\
\hline SAG 51.72 & Chloromonas rosae & Ch & +++ & $198^{\mathrm{C}}$ & 3.0 & 3.0 & $14.6^{\mathrm{f}}$ \\
\hline$\overline{\text { SAG 31-1 }}$ & Chlamydocapsa maxima & $\mathrm{Ch}$ & n. r. & 155 & 0.7 & 0.7 & n.r. \\
\hline SAG 12-2a & Chlorogonium elongatum & Ch & n. r. & 370 & 0.0 & 0.0 & n.r. \\
\hline
\end{tabular}

*Strains used for three cycles of cryopreservation (see Table 5.2).

apost-thaw viability levels from very poor $(+)$ to very good $(++++)$, with intermediates for very poor $(+)$, poor $(++)$ and good regrowth $(+++)$. Two samples did not regrow (n.r.).

${ }^{\mathrm{b}}$ Absolute number of evaluated AFLP fragment positions using EcoRI+A/MseI+C,

EcoRI+C/MseI+C, EcoRI+G/MseI+C or ${ }^{\mathrm{c}}$ different primer combinations (see text).

${ }^{\mathrm{d}}$ Expressed as percentage.

${ }^{\mathrm{e}} \mathrm{AFLP}$ reaction was non-reliable.

${ }^{\mathrm{f}}$ Strain was not axenic after cryopreservation. 


\subsubsection{AFLP results after cryopreservation}

\section{AFLP results of the cultures directly after thawing}

After cryopreservation, cultures from each of the 29 strains were thawed, diluted with fresh medium and allowed to recover $12 \mathrm{~h}$ in darkness. DNA was then extracted directly and investigated with AFLP. The post-cryo patterns obtained were manually compared with the three pre-cryo patterns from each strain to ascertain whether there were differences in banding patterns before and after cryopreservation. Only fragments of reproducible fragment positions were counted. These post-cryo patterns were identical with the ones obtained before cryopreservation for 19 strains, as shown exemplarily in Fig. 5.1 for Macrochloris radiosa, in which no differences were obtained directly after thawing (electropherogram D). For the remaining ten samples, an average of $0.3 \%$ of all compared reproducible fragment positions (ranging from 0.0 to 3.0\%) were different (Table 5.1). Banding patterns of the later, nonregrown strains of Chlamydocapsa maxima and Chlorogonium elongatum showed $0.7 \%$ and no differences directly after thawing (not shown).

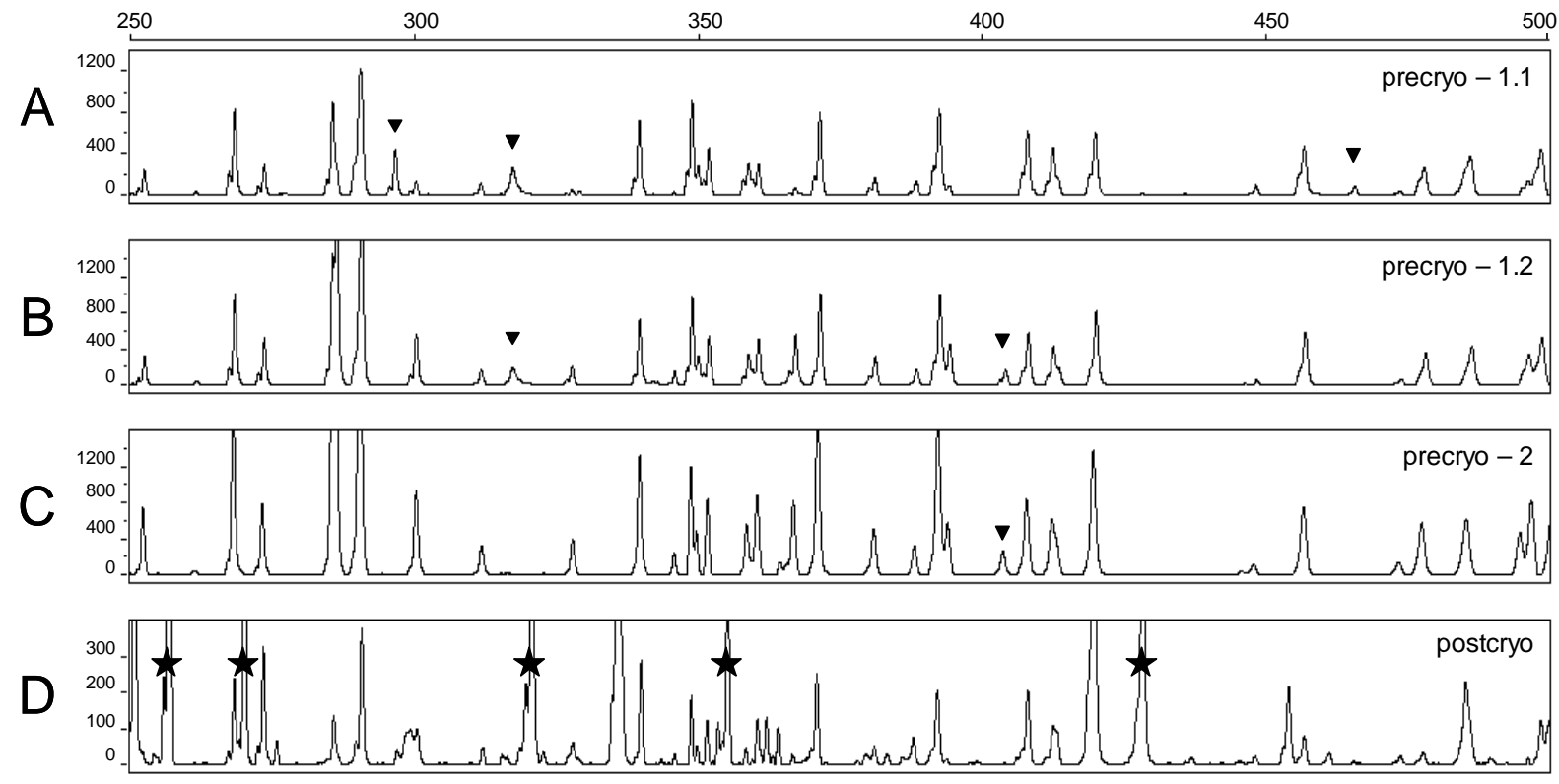

Fig 5.2 AFLP patterns of Chloromonas rosae SAG 51.72 before (A-C) and after cryopreservation (D). Arrowheads denote non-reproducible fragments within the three pre-cryo banding patterns $(A-C)$. Asterisks denote additional fragments in the post-cryo banding pattern of the regrown culture due to contaminants (D). Electropherograms from selective amplification with primer combination, EcoRI+C I Msel+CG. Vertical scales, relative fluorescent units; horizontal scales, size of fragment in nucleotides.

\section{AFLP results of the regrown cultures}

Two weeks after thawing and culturing under standard conditions, 27 of the 29 strains under study had survived and viability levels ranging from very poor $(+)$ to very good $(+++$ + ) were obtained (Table 5.1). DNA was extracted from each of these regrown cultures and 
subsequent AFLP analyses resulted in the post-cryo banding patterns of the regrown culture. These banding patterns were manually compared with the pattern obtained post-cryo directly after thawing and with the three pre-cryo patterns. Differing fragments were only counted if they were in reproducible fragment positions (see above). Additional post-cryo fragments of a regrown culture are shown for Macrochloris radiosa (Fig. 5.1, denoted with arrows) or for Nostoc sp. (Fig. 5.3, within boxes). Comparison of pre- and post-cryo patterns provided a total number of fragment positions that included both the number of all non-reproducible fragments before cryopreservation and the number of differing post-cryo fragments (directly after thawing and of the regrown culture). The quantity of differing post-cryo fragments, derived from the regrown cultures, was counted and calculated as a percentage of all fragment positions. No differences were found for ten strains (Table 5.1). For 17 strains, differences ranging from 0.5 to $14.6 \%$ (mean $1.3 \%$ ) were obtained. As within the pre-cryo banding pattern, the post-cryo banding patterns also exhibited fragments with low peak intensities (mean $0.4 \%$ ). In general, the differing post-cryo fragments directly after thawing were apparently identical to those found in the patterns of the regrown samples. In the following section of the results, post-cryo patterns accordingly refer only to the patterns of the regrown cultures.

Amphikrikos sp. with $10.1 \%$ differing fragments and Chloromonas rosae with $14.6 \%$ were excluded from the average value of post-cryo differing fragments. The high level of $14.6 \%$ post-cryo differences in Chloromonas rosae consisted of $13.1 \%$ additional and $1.5 \%$ missing fragments. It was assumed that these additional fragments were likely generated by contaminant organisms. This was subsequently supported by a positive contaminants test using an enriched culture medium, although the contaminants were not visible in the regrown culture. The influence of the contaminant on the banding pattern is shown in Fig. 5.2 where asterisks denote some of the fragments considered to be generated by contaminant DNA. Their influence on the patterns was obvious and resulted in an atypical pattern for this strain.

The second strain that had unusually high levels of post-cryo differences (8.5\%), was Amphikrikos sp. The post-cryo AFLP analysis was repeated and resulted in the same high level of differing fragments. This finding supported the result, also made before cryopreservation, of a non-reliable AFLP reaction. Failure in the AFLP reaction was almost certainly due to poor DNA quality (data not shown). 


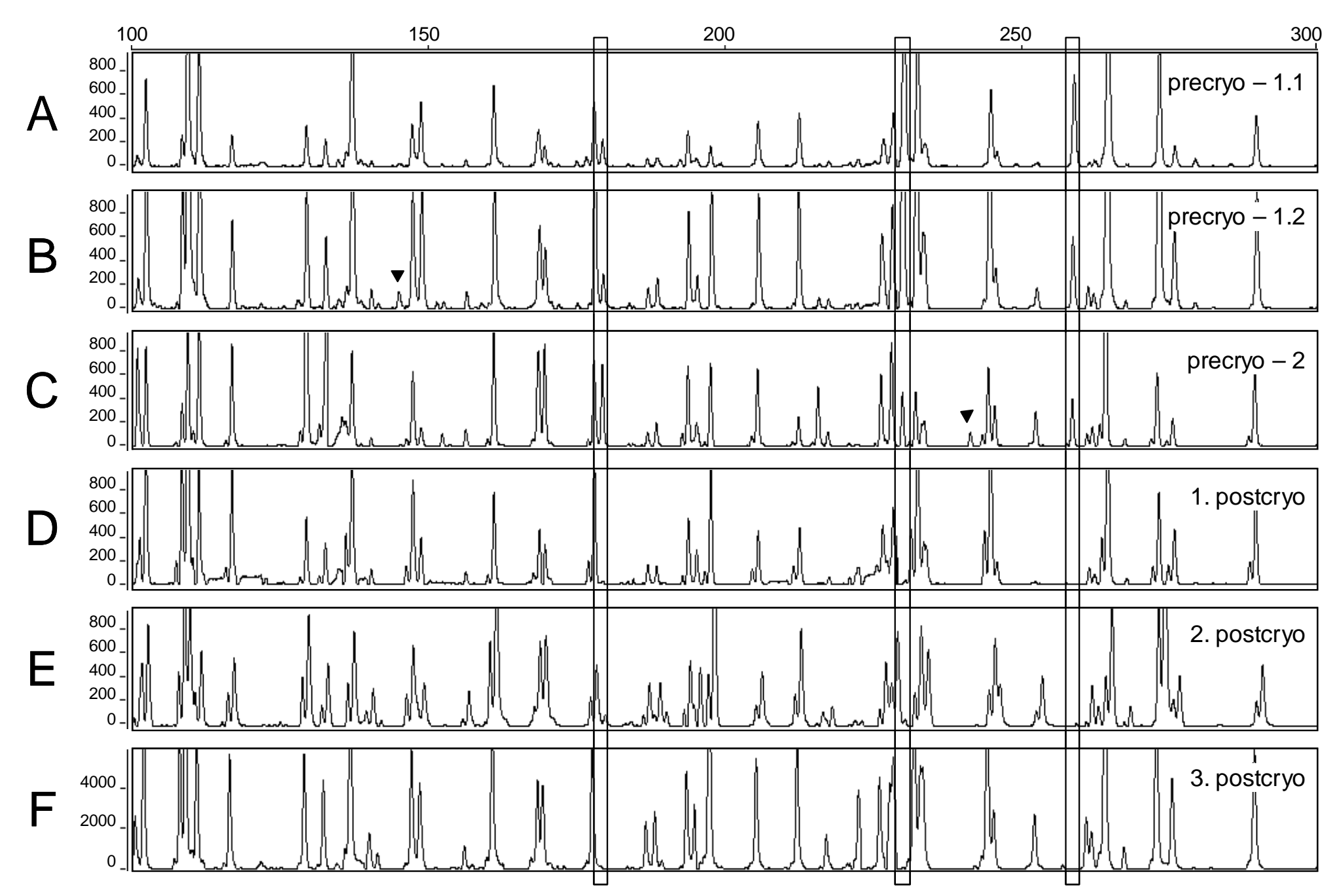

Fig. 5.3 AFLP banding patterns of Nostoc sp. PCC 7120 before (A-C) and after the first, second and third cycle of cryopreservation (D-F). All post-cryo patterns were obtained from the regrown culture. Arrowheads denote non-reproducible fragments. Fragment positions with post-cryo differences are within boxes. Electropherograms from selective amplification with one primer combination, EcoRI+A/Msel+C, Vertical scales, relative fluorescent units; horizontal scales, size of fragment in nucleotides. 


\subsubsection{Results for the three cycles of cryopreservation}

Stable AFLP banding patterns were obtained for three of the six strains after the third cycle of freezing and thawing (Table 5.2). It is worthy of note, that the DNA in this experiment was only investigated from regrown cultures and not directly after thawing. For Chlorella vulgaris, an identical additional fragment was obtained after the second and the third cycle. In Nostoc sp. three fragments were missing after the first cycle and they remained absent after the second and the third cycle. Furthermore, after the third cycle an additional fragment was encountered. The three missing fragments are shown in Fig. 5.3 within boxes. Five strains showed good or very good post-thaw viability (PTV) levels after each freezing and thawing cycle (Table 5.2). Euglena gracilis had poor and very poor viability levels after the first and second cycle and no viability was observed two weeks after thawing following the third cycle. However, E. gracilis cultures recovered after each of the three cycles after four to five weeks. AFLP banding patterns of Euglena gracilis were stable after the first and second cycle, but had a missing fragment after the third cycle for which the DNA was extracted from the four-week-old culture (Table 5.2).

\subsubsection{Comparison of genetic analyses and PTV levels}

In general, PTV levels obtained did not correlate with the amount of differing postcryo fragments (Table 5.1). Strains with very good PTV levels belonged to the group with and without differences in post-cryo patterns. However, it was apparent that the group of strains without post-cryo differing fragments consisted without exception of strains with good or very good PTV levels.

Table 5.2 Strains used for three cycles of freezing and thawing. Listed are the amount of nonreproducible fragments before cryopreservation and the PTV levels and AFLP results after each cycle, see text.

\begin{tabular}{lccccccc}
\hline \hline & \multicolumn{2}{c}{$1^{\text {st }}$ cycle } & \multicolumn{2}{c}{$2^{\text {nd }}$ cycle } & \multicolumn{2}{c}{$3^{\text {rd }}$ cycle } \\
\hline & $\begin{array}{c}\text { AFLP non- } \\
\text { repr. pre- } \\
\text { cryo }^{\mathrm{a}}\end{array}$ & PTV $^{\mathrm{b}}$ & $\begin{array}{c}\text { AFLP } \\
\text { diff. post- } \\
\text { cryo }^{\mathrm{a}}\end{array}$ & PTV $^{\mathrm{b}}$ & $\begin{array}{c}\text { AFLP } \\
\text { diff. post- } \\
\text { cryo }^{\mathrm{a}}\end{array}$ & $\begin{array}{c}\text { AFLP } \\
\text { PTV }^{\mathrm{b}}\end{array}$ & $\begin{array}{c}\text { diff. post- } \\
\text { cryo }^{\mathrm{a}}\end{array}$ \\
\hline Chlamy. reinhardtii & 0.9 & +++ & 0.0 & +++ & 0.0 & +++ & 0.0 \\
Chlorella vulgaris & 3.0 & ++++ & 0.0 & +++ & 0.8 & +++ & 0.8 \\
Cosmarium cucumis & 0.0 & ++ & 0.0 & ++ & 0.0 & ++ & 0.0 \\
Euglena gracilis & 0.0 & ++ & 0.0 & + & 0.0 & - & 0.3 \\
Phaeod. tricornutum & 0.6 & +++ & 0.0 & +++ & 0.0 & ++++ & 0.0 \\
Nostoc sp. & 1.6 & +++ & 1.6 & +++ & 1.6 & ++++ & 2.1 \\
\hline
\end{tabular}

bexpressed as percentage.

apost-thaw viability levels from very poor $(+)$ to very good $(++++)$, with intermediates for very poor $(+)$, poor $(++)$ and good regrowth $(+++)$. One sample did not regrow after two weeks (-). 


\subsection{Discussion}

\subsubsection{Reproducibility of AFLP patterns}

The level of non-reproducible fragments obtained here before cryopreservation, with a maximum of $3.6 \%$ and mean $0.9 \%$, was found to be consistent with previous reproducibility tests of AFLP in plants (Hansen et al. 1999), microalgae (John et al. 2004) and animals (Bonin et al. 2004) where rates below 5\% were obtained. In these publications different DNA extractions were also used for reproducibility testing and these were found to be essential for this purpose. This was supported by AFLP analyses of mutants of Parachlorella kessleri where four replications were performed per strain. The comparison of these replications proved to be an adequate and reliable tool to identify non-reproducible fragments with the objective of increasing the accuracy of the AFLP results (Chapter 3).

The reproducibility levels obtained here confirmed the successful application of AFLP to a variety of microalgal strains using an identical, standardized protocol with only slight variations in the choice of selective nucleotides for selective amplification. The number of fragments obtained using this standard protocol varied and differences were thus calculated as a percentage to obtain comparable results between strains. It was not the objective to test which primer combinations resulted in a high number of fragments per primer combination or to obtain as much data per combination as possible. The protocol used here provides the basis for future AFLP analyses in microalgae. For extended AFLP analyses within one species, it might be advisable to test more primer combinations to optimize the number of amplified fragments per primer combination in selective amplification.

\subsubsection{Reasons for non-reproducible fragments}

The amount of non-reproducible fragments found in this study was relatively low and in the same range as found in other publications (see above). The question what caused these non-reproducible fragments remains unanswered. It was assumed in this study that nonreproducible fragments can be due to technical differences (artifacts, errors during the AFLP procedure) or non-homogenous algal cultures (presence of contaminants or subpopulations). The presence of contaminants would lead to a high amount of differences, e.g., above $14 \%$ as was demonstrated for Amphikrikos sp., and can therefore be easily identified. Influence of subpopulations is more critical for the post-cryo investigations because the subpopulations can differ in their sensitivity to cryopreservation and cryo-selection can potentially occur (Pearson et al. 1990). An altered ratio of subpopulations would then lead to differences in post-cryo patterns. For pre-cryo analyses the influence of putative subpopulations on the 
patterns should be smaller; the aspect of subpopulations is discussed in Chapter 5.5.4. It is more likely that the non-reproducible fragments are primarily due to technical differences, which can be expected for a technique as complex as AFLP. In this study the amount of nonreproducible fragments before cyopreservation represents the error rate of the AFLP technique.

\section{Technical differences}

The level of non-reproducible fragments within the three pre-cryo replicates (mean $0.9 \%$ ) is comparable with the value of $1.3 \%$ of technical (artifactual) differences (e. g. differences in peak intensities, restriction anomalies) found by Bonin et al. (2004) within replicates of Betula nana samples. A certain error rate can be anticipated for any restriction or PCR-based technique owing to non-selective restriction or amplification (e.g. Koonjul et al. 1999). In the following, the influence of non-selective amplification and non-selective restriction due to poor DNA quality will be discussed.

The amount of differences produced by the amplification is difficult to determine, but an estimation may be given by the amount of fragments of low intensity close to the background that are likely generated by non-selective amplification (Bonin et al. 2004). In this study they represented $0.3 \%$ of all non-reproducible fragments. In addition, technical differences are affected by the purity and quality of DNA, which has been found to be the limiting factor in obtaining high quality AFLP fingerprints (McLenachan et al. 2000, Mannschreck et al. 2002, Bonin et al. 2004) and influences the quality of the digest. This finding is supported in this study by the observation that non-reproducible fragments were mainly found between patterns of the two different DNA extractions and not within the two patterns of the same DNA extraction. Often patterns of the same DNA are more similar to each other than to the pattern of another DNA extraction (Fig. 5.1). The influence of poor DNA quality, which results in weak and non-reliable selective amplification, was demonstrated for Amphikrikos sp. with 8.5\% differences (Table 5.1), and was clearly higher than the maximal amount of 3.6\% non-reproducible fragments for the other strains studied here. For this strain it was known that the DNA extraction is critical and sometimes yielded DNA that could not be amplified by PCR (Hepperle et al. 2000). The same problem seemed to have occurred in this study and resulted in a high level of non-reproducible fragments. 


\subsubsection{AFLP patterns after cryopreservation}

After cryopreservation an average of $1.3 \%$ differences were obtained, ranging from 0.5 to $6.3 \%$ (Table 5.1) and for three of the six strains subjected to three cycles of cryopreservation differences ranged from 0.3 to $2.4 \%$ (Table 5.2). These differences were only counted for reproducible fragment positions, i.e. the non-reproducible fragments (mean $0.9 \%$ ) were excluded. Only the differences of the regrown culture are discussed, since the differences found directly after thawing were identical with them and the differences of the regrown culture represented higher levels (Table 5.1). The post-cryo differences found in this study were higher than differences obtained in AFLP investigations after cryopreservation in higher plants. In Prunus $0.4 \%$ of the fragments were found to be variable, which was observed more often after cryopreservation than in non-cryopreserved samples (Helliot et al. 2002). In strawberry, one post-cryo missing fragment was observed in one of 16 investigated primer combinations (Hao et al. 2002). No post-cryo differences were found in the relatively high number of 433 fragments evaluated in the Asteraceae, nor in the smaller number of 95 AFLP fragments examined in the Haemodoraceae, or in the 196 fragments in apple shoots (Turner 2001, Wilkinson et al. 2003, Liu et al. 2004). In this study, between 127 and 371 fragments were obtained; this is in the same range as in the cited investigations for higher plants. The higher level of post-cryo differences observed in microalgae might be explained by the higher morphological and physiological diversity present in the investigated strains. In all studies of the higher plants mentioned above, samples of the same species or genus were analysed. The response to cryopreservation of samples of the same species or genus might be more uniform than the response of the different microalga strains. Reasons for these post-cryo differences are discussed in more detail below.

\subsubsection{Reasons for the post-cryo differences}

Post-cryo differences in banding patterns were observed for 15 strains tested in this study (Table 5.1 and 5.2). These differences were in addition to the error rate obtained prior to cryopreservations. Therefore these different fragments represent real, additional differences found after cryopreservation. Reasons for these differences include the possible presence of contaminants, subpopulations, DNA methylation or cryoinduced genetic changes (see below). 


\section{Contaminants}

Generation of post-cryo differences due to a contaminant can be excluded here because axenity of strains was tested and it was found that a contaminant would produce much higher amounts of differences than obtained here, with in average $0.9 \%$ and maximum of $6.3 \%$. The amount of differences produced by contaminants was demonstrated for Chloromonas rosae, with 14.6\% (Table 5.1, Fig. 5.2). The amount of artificial differences produced if the contaminants remain undetected is thus evident. In AFLP analyses of fungi, a bacterial contaminant was reported to have almost formed a 'unique genotype' by the presence of additional fragments and no missing fragments were found (Dyer and Leonard 2000). For Chloromonas rosae $13.1 \%$ of the post-cryo differing fragments were additional and $1.5 \%$ were missing. These missing fragments were probably due to a suppression of alga-specific fragments by DNA of contaminating organisms during DNA amplification, as it was found in Chapter 4 for strains of Dunaliella salina. The putative influence of small contaminations, e.g. single bacteria, on the banding pattern cannot be excluded completely, even if any precautions were taken during DNA extraction, restriction/ligation and PCR reactions to avoid contaminations (e.g., sterile conditions on a clean bench). Nonetheless, potential contamination influence is already taken into account in the reproducibility tests performed before cryopreservation and thus represents part of the error rate of the technique.

\section{Subpopulations and cryo-selection}

If an algal culture is genetically non-homogenous and consists of different subpopulations, these subpopulations can exhibit differing degrees of sensitivity to cryopreservation. It is possible that not all subpopulations will survive the cryopreservation process or that one subpopulation will dominate the post-cryo culture. Both cases result in cryo-selection and will lead to differences in post-cryo AFLP banding patterns.

With respect to the putative presence of subpopulations, all cultures in this study should have been derived from a single cell (Schlösser 1994) and thus represent a genetically homogenous culture. However, there is also potentially reason to expect the opposite. In a theoretical overview of rates of spontaneous mutations found in studies of a broad range of organisms, Drake et al. (1998) suggest that for asexual organisms the rate of recombination could be higher than is commonly supposed and so their mutation rates have evolved similar values to those of sexual species. Furthermore, in algal populations it is suggested that preselective mutations are sufficiently frequent to allow them to adapt to extreme natural environments (Flores-Moya et al. 2005). A third factor is the serial transfer in which all 
strains have been maintained for many decades, which also adds selective pressure and/or genetic drift on the organisms (Day and Brand 2005). These three considerations, taken together, make it not unlikely that the cultures involved are genetically heterogeneous and that subpopulations are present. In this study the influence of subpopulations on the AFLP banding patterns before cryopreservation is included in the error rate estimated for the nonreproducible fragments between DNA extractions (Chapter 5.5.2). In these DNA extractions the ratio of DNA derived from single subpopulations may vary and can lead to nonreproducible fragments. This was observed in a preliminary study on Chlorella vulgaris, in which non-reproducible fragments were found between DNA extractions of the same culture (cf. Chapter 2). Single cells of this culture were isolated, regrown and used for DNA extraction; subsequent AFLP analyses resulted in patterns in which the non-reproducible fragments were only present in a few or one of the clones (data not shown). This suggests the presence of subpopulations within the Chlorella vulgaris culture, although further investigations are necessary to verify this hypothesis.

A greater influence of subpopulation on the AFLP results can be expected after cryopreservation (see above). Subpopulation-specific differences could result in cryoselection of only a part of the putatively heterogeneous culture. It has been suggested that high PTV levels, i.e. above $60 \%$, are optimal to guarantee a representative, successfully cryopreserved and genetically stable culture (Day and Brand 2005). Two of the six strains used in this study for three successive cycles of cryopreservation did not reach this level, with only poor or very poor PTV (Table 5.2). For Cosmarium cucumis this was not correlated with differences in AFLP patterns. For Euglena gracilis one differing fragment was found after the third cycle and might represent a difference caused by cryo-selection of a dominant subpopulation after the third cycle. For E. gracilis it has previously been observed that cryopreservation success can differ, even when using an identical cryopreservation procedure (Day 1998). For the other five strains PTV levels were reproducible (Table 5.2). For the strains that were cryopreserved once, no correlation was found between the PTV levels and the post-cryo genetic differences, but it was obvious that all strains without post-cryo differences exhibited good or very good PTV (Table 5.1). This might support the hypothesis of possible cryo-selection. On the other hand, strains with very good PTV revealed differences in their post-cryo AFLP patterns, e.g. Uronema belkae or Scenedesmus obliquus (Table 5.1), indicating that cryo-selection is not the only explanation for post-cryo differences. 


\section{DNA Methylation}

Changes in DNA methylation status can be an adaptive response to high osmotic stress (e.g., Turner et al. 2001) and can therefore be influenced by the cryopreservation process of freezing, thawing and recovery. Post-cryo differences in restriction-enzyme-generated fingerprints have been explained to be likely due to a changed DNA methylation status: $0.3 \%$ fragment differences were reported by Helliot et al. (2002) and Harding (2004). They suggested that these differences were because restriction enzymes such as EcoRI cannot cleave methylated restriction sites. For strawberry and apple a higher level of DNA methylation was detected after cryopreservation, using a modified AFLP technique known as methylation-sensitive amplified polymorphism (MSAP, Hao et al. 2001, Hao et al. 2002). Since DNA methylation status can change over a short timescale and in response to environmental stimuli (Jarvis et al. 1992), the two weeks of recovery after cryopreservation were possibly not enough time for the microalgae to reach the DNA methylation status of prior to freezing. Little is known about this topic in microalgae. DNA methylation status of Chlorella vulgaris 211-11b two months after cryopreservation was measured by K. Harding (pers. comm.) and revealed 13\%. This was an unexpectedly high level, especially in comparison to different microalgae investigated by Jarvis et al. (1992), who detected levels of about $2 \%$ and maximal values of $11 \%$. Thus, DNA methylation is a likely explanation for at least a part of the post-cryo fragment differences identified in the this study. After cryopreservation the SAG strain of Coccomyxa arvernensis had an additional fragment in its post-cryo pattern (0.5\% differences, cf. Table 5.1). This additional fragment was also present in the $2.8 \%$ differing postcryo fragments of the CCAP strain, but it was not present in any of the precryo patterns obtained from these two duplicate strains (data not shown). Perhaps the corresponding post-cryo additional fragment found in both duplicate strains was caused by a corresponding change in DNA methylation status. However, future analyses of genetic stability using AFLP should include a parallel assessment of DNA methylation status.

\subsubsection{Cryoinduced genetic alterations}

Reasons for cryoinduced genetic alterations are manifold and complex in their interactions. They include the formation of free radicals, damaged enzyme functions and the influence of the cryoprotectant. In this study there was evidence that some of the post-cryo differences obtained were cryo-induced, because they were already observed in the culture directly after thawing (Table 5.1). These differences were only counted on reproducible fragment positions and may present a change in DNA methylation status within the twelve 
hours of recovery, or a mutation induced by the cryoprocess itself. Influence of cryo-selection can be excluded in the short time of recovery and in addition DNA was extracted from the whole culture including both live and dead cells. For the two samples that did not regrow, Chlamydocapsa maxima had one differing fragment $(0.7 \%)$ and Chlorogonium elongatum no differences. The reason why they did not survive the cryoprocess was thus unlikely to be due to lethal mutations directly introduced by the cryoprocess itself, but due to lethal freezeinduced injuries (Fleck et al. 1997). On average, 0.3\% differences were observed directly after thawing in ten of the 27 samples (Table 5.1) and all these differences were also present in the banding patterns of the regrown cultures. During the enormous stress of cryopreservation, mutations can be induced by free radicals, which are formed under normal conditions, but controlled by complex metabolic interactions; under stress the protection mechanisms become saturated (Benson 1990). Once produced, the free radicals can initiate the formation of further radical species and under certain circumstances DNA can come under free radical attack, and alterations to the genome may occur (for details see Benson 1990). In addition large temperature variations can influence enzyme functions, which can be lost or inactivated, and lead to inefficient DNA repair systems (Calcott and Gargett 1981). Cryoprotectants help to avoid these problems, but DMSO is for example known to be mutagenic (Vannini and Poli 1983). However, penetrating cryoprotectants, including DMSO, can also act as free radical scavengers and therefore protect the DNA (Benson 1990). The effect of background natural ionizing radiation may be negligible in the present study because the cultures were all investigated within a time period of one week. However, this may have a significant influence on long-term exposures. These few examples illustrate the complexity of all processes acting together and that not all influences could be addressed here.

\subsection{Conclusions with respect to the differences obtained after cryopreservation}

Genetic differences observed for 15 of the 27 strains in this study after cryopreservation do not represent errors of the AFLP technique itself. The error rate was already subtracted from these values by evaluation of non-reproducible fragments before cryopreservation in reproducibility tests. The post-cryo differences obtained were assumed to be genetic alterations after cryopreservation that were due to changed DNA methylation status, cryo-selection or cryoinduced mutations caused e.g. by free radicals or DMSO. Contaminants could be excluded as an explanation for these differences. For seven strains, namely Trebouxia sp., Chlamydomonas moewusii, Pseudendocloniopsis botryoides, 
Coccomyxa arvernensis, Uronema belkae, Coelastrum morum and Scenedesmus obliquus the level of post-cryo differences was in the same range as differences found between pigment mutants and wildtype of Parachlorella kessleri. These mutants were induced by UV light or radioisotopes and have clearly different phenotypic properties compared to their wildtype. Obviously, if these mutations would have appeared after cryopreservation, these strains could not be considered to be successfully cryopreserved. The genetic differences observed here should therefore be investigated by an extended use of physiological and biochemical characters, e.g. photosynthetic activity, vital staining, and fluorescence measurements (for review see Taylor and Fisher 2003) to establish whether the differences on the molecular level are correlated with differences on the phenotypic level. To assist in identifiying the influence of changes in DNA methylation status, DNA methylation measurements should be performed. Furthermore, it is worth noting that even the strains without detected post-cryo differences could potentially have small post-cryo differences in their genome. In any fingerprinting technique, the detection of mutations is governed by chance. However, AFLP is one of the best techniques available to investigate genetic stability. The genetic integrity after cryogenic storage can be investigated on the phenotypic, histological, cytological, biochemical and molecular levels (cf. Introduction, Harding 2004). In this context, molecular investigations are especially useful for microalgae, which due to their small size and lack of satisfactory morphological characters are often difficult to characterize on the other levels. The AFLP technique was found to be a reproducible method to investigate a wide range of microalgae, and this study has layed the basis for future AFLP analyses in this field. 


\subsection{AFLP reproducibility tests between European laboratories}

\section{Introduction}

An AFLP protocol was developed in this study to investigate the genetic stability of microalgae after cryopreservation. In course of the European Resarch project COBRA a validation of this protocol was performed within the consortium of different culture collections of algae (CCAP culture collection, abbreviation NERC and SAG culture collection, abbreviation Uni-Goe) and also external validation was undertaken by two European Biological Resource Centres (ALGOBANK, Caen, France \& CABI Bioscience, Egham, UK). The main objective was to investigate the usefulness of the AFLP technique in the context of cryopreservation and to report to the wider culture collection community the outcomes of the assessments. A high reproducibility (90-98\%) of AFLP fragments has been demonstrated between different laboratories (Jones et al. 1997), but it has been criticized that this study used the same original DNA sample in the replicates (Donaldson et al. 2000). Therefore a comparison of AFLP reactions performed by different laboratories starting with DNA extractions using the same strain was needed to provide more sound data on the reproducibility of the AFLP method. The results of such a reproducibility test are shown in this study.

\section{Validation procedure and preliminary results}

Seven strains of Chlorella vulgaris were selected with two additional duplicate strains from CCAP for validation of the AFLP genotypic stability testing method: SAG 211-11b, SAG 30.80, SAG 211-11j, SAG 211-12, SAG 211-11p, CCAP 211/11J and CCAP 211/12. These strains represented the five different AFLP patterns that were obtained for $C$. vulgaris (Chapter 2). For strain SAG 211-11b the AFLP procedure was performed before and after cryopreservation (two to three weeks after thawing and regrowth). For each sample two replicates were made using a first DNA extraction and the third using a second DNA extraction of the same culture, resulting in a total of 24 AFLP analyses. Replications were found to be essential to identify artefactual, non-reproducible fragments (Chapters 2 and 5.5), and in addition provided a reproducibility test of each partner. Electrophoresis of all AFLP reactions was performed on a capillary sequencer at SAG culture collection (Uni-Goe). Electrophoresis on the same sequencer was found to be essential to enable reliable comparison of banding patterns. Complete evaluation of banding patterns was part of this study. In May 2005 all 24 analyses were successfully conducted by partners CCAP culture 
collection (NERC), ALGOBANK and Uni-Goe. CABI sent some first samples and will send the remaining ones in the next couple of weeks.

In a questionnaire that was sent to all evaluators it was double-checked that all evaluators had used comparable AFLP procedures. The AFLP technique was found to be very reproducible within the replications of one partner as well as between partners. An example of the banding patterns obtained for SAG 30.80 from NERC, Uni-Goe and ALGOBANK is demonstrated in Fig. 5.4. In evaluating the patterns the height of the fragments is not informative, it is only counted if a fragment is present or absent. Denoted are additional fragment positions of NERC or ALGOBANK. All evaluated samples revealed as high reproducibility as shown for SAG 30.80. Patterns of the different isolates could clearly be identified. Banding patterns of all duplicate strains were found to be virtually identical. Comparison of patterns before and after cryopreservation resulted in no differences if nonreproducible fragments were excluded from the analyses (cf. Chapter 5.3.4). As an example, pre- and postcryo patterns from ALGOBANK are shown in Fig. 5.5. Two non-reproducible fragment positions are shown within boxes, this points highlights the importance of replications to identify non-reproducible fragments (cf. e.g. Chapter 3.4.2). Final evaluation of all AFLP samples has to be performed manually as soon as the samples from CABI are available. This evaluation will lead to percentage values and a statistic evaluation of the results. It is aimed for to publish this reproducibility test as soon as possible.

The use of reagents from different manufacturers had no influence on the reproducibility of patterns, e.g. only ALGOBANK and Uni-Goe used the same kit for DNA extraction and NERC tested enzymes from two different manufacturers. This is an important result, because the objective was to develop an AFLP protocol independent of manufacturers. Troubleshooting of the protocol had been undertaken by NERC, ALGOBANK and CABI and the final version of the AFLP protocol will be published together with other validation protocols by the COBRA consortium soon.

It was demonstrated during this validation exercise that the AFLP protocol developed was suitable to reveal reproducible banding patterns. It is particularly impressive that the exercise has been so successful considering that prior to the project molecular biology expertise/experience of the validators was very different. It ranged from no expertise at ALGOBANK to very good expertise of CABI and Uni-Goe, with intermediate levels of expertise and experience at NERC. The visit exchanges of partners were very helpful in initiating the program of work and insuring partners conformed to the protocols, this approach is recommended for future validation exercises. However, ALGOBANK established the 
AFLP technique in their lab without any visits and this was facilitated by close E-mail contact between ALGOBANK and Uni-Goe. In summary, the AFLP technique was found to be reliable and reproducible within individual labs and between validating labs. This was the first time that the reproducibility of the whole AFLP procedure from DNA extraction to selective amplification was tested between different laboratories.
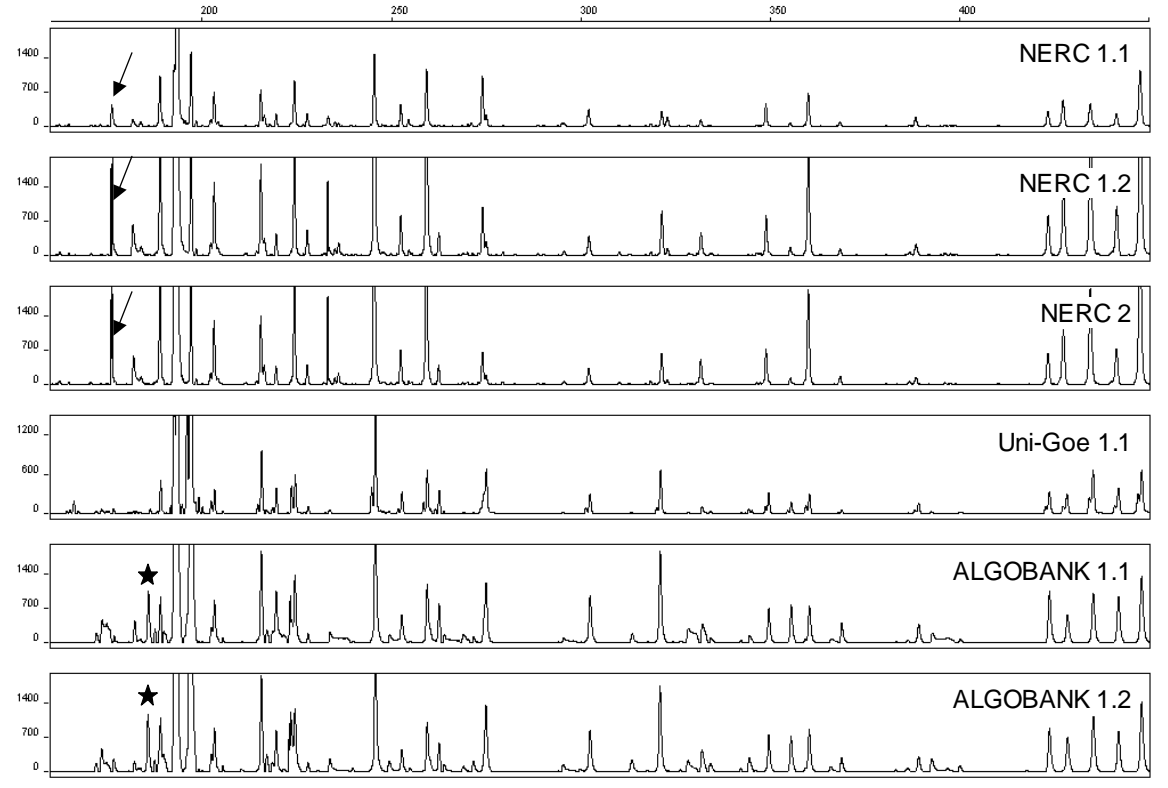

Fig. 5.4 AFLP electropherograms of SAG 30.80 performed by NERC, Uni- Goe and ALGOBANK. Arrows denote an additional fragment postion of NERC patterns. Asterisks denote an additional fragment postion of ALGOBANK patterns. Primer combination EcoRI+C/Msel+C. Vertical scales, relative fluorescent units; horizontal scales, size of fragment in nucleotides.

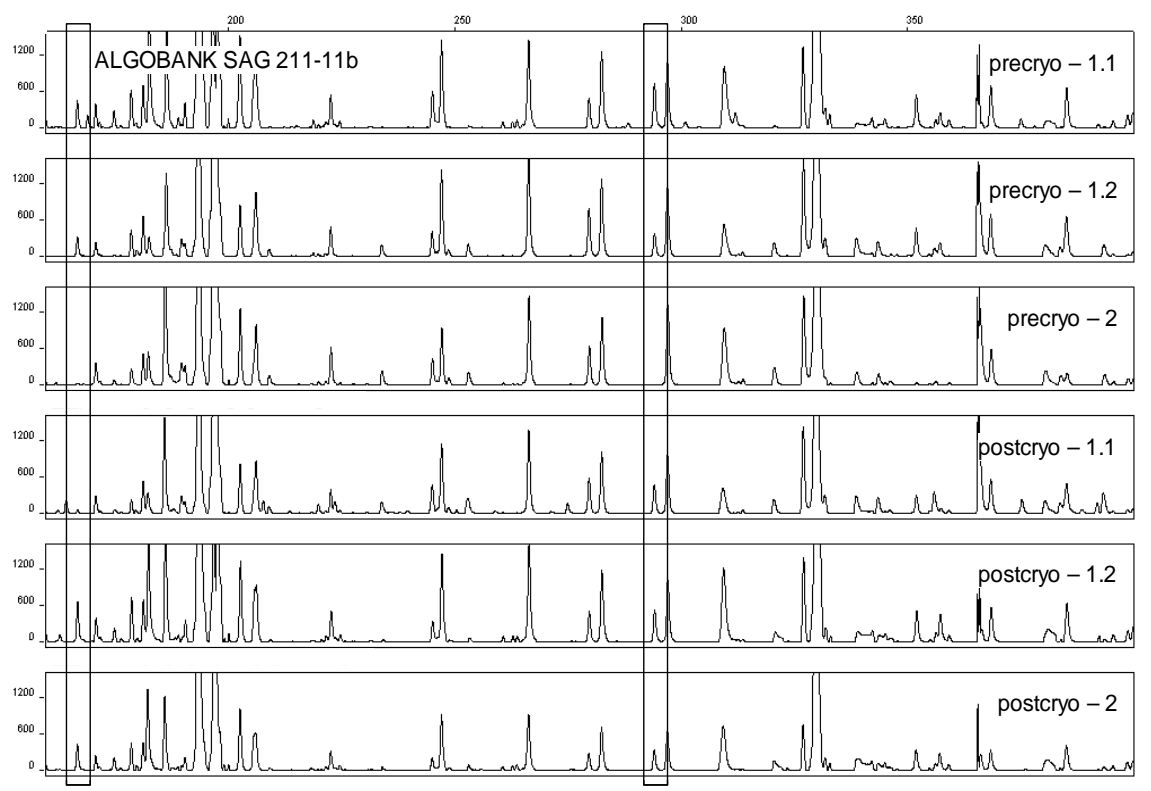

Fig. 5.5 AFLP electropherograms of SAG 211-11b before and after cryopreservation performed by ALGOBANK. Non-reproducible fragment postions are within boxes. Primercombination $\mathrm{EcoRI}+\mathrm{C} / \mathrm{Msel}+\mathrm{C}$ Vertical scales, relative fluorescent units; horizontal scales, size of fragment in nucleotides. 


\title{
6 Detection of a viral infection in Chlorella and analysis of host ranges using amplified fragment length polymorphism
}

\begin{abstract}
6.1 Abstract
Viral infections of microalgae are known for a considerable number of taxa. Infection can follow one of two basic cycles, i.e. being lytic or latent. The most common type is lytic infection, which results in loss of the culture. Owing to their small size of 20 to $400 \mathrm{~nm}$, viruses are difficult to detect; their genome size can be up to $510 \mathrm{kbp}$. In this study it was shown that the AFLP technique is well suited to detect viral infections in cultures of microalgae. AFLP patterns of any algal culture can be screened for the presence of virus-specific patterns. The method is not restricted to axenic cultures, does not require comparison with an uninfected culture and has the potential to detect novel viruses. As test organisms, Chlorella sp. NC64A and the virus PBCV-1 were investigated. NC64A was isolated from Paramecium bursaria collected in the United States ca. 45 years ago. Sequence analyses of the $18 \mathrm{~S}$ and ITS rDNA regions showed identical sequences for NC64A and two isolates that were isolated from P. bursaria collected in Japan. In addition, the Japanese isolates were also sensitive to PBCV-1. These findings support previous hypotheses that viral infection experiments provide a useful tool for classifying species of algae. However, using AFLP, differences were found between the American and the Japanese isolates. Consequently, with AFLP it was possible to distinguish isolates within the host range of the same virus.
\end{abstract}

\subsection{Introduction}

The existence of viruses in algae has been known since they were first reported in the 1960s and early 1970s (Van Etten et al. 1991). By 1991, at least 44 taxa of eukaryotic algae had been observed to host algal viruses (Van Etten et al. 1991), and since then the number of documented virus occurrences in eukaryotic algae and cyanobacteria has grown considerably (Lawrence 2005). Cyanophages are viruses that infect cyanobacteria. A total of three families are known and all contain double-stranded DNA (dsDNA). Currently, only one family of eukaryotic algal viruses is known, of which all members contain dsDNA. In addition, a smaller number of viruses are known that do not fit into this family and contain singlestranded RNA (ssRNA). Viral infection can follow one of two basic cycles, being lytic or latent, with lytic infections being the most common. The existence of chronic or persistent 
infections in algae is currently a matter of debate (Lawrence 2005). For extensive reviews on algal viruses, see Lawrence (2005) and Van Etten et al. (1991).

Viruses can lead to difficulties in algal culturing, particularly if the viruses cause culture lysis and the culture is lost (see Fig. 1.2d). Due to their small size of only 20 to 400 $\mathrm{nm}$, viruses are difficult to detect in algal cultures; their genome size can be up to $510 \mathrm{kbp}$. Non-lytic viruses can thus be problematic in molecular and genomic studies, because they contaminate the algal culture with their DNA. The latter point was investigated in this study using the fingerprinting technique amplified fragment length polymorphism (AFLP). It was previously shown that different genotypes within one species can be distinguished using this technique (Chlorella vulgaris, Chapter 2), and that atypical, artificial genotypes are obtained when a contamination of bacterial and/or fungal origin is present (Chapters 4 and 5). In contrast to bacterial/fungal contaminations, viral contaminations cannot be detected by microscopic inspection or tests like culturing on organically enriched culture media (e.g. Chapter 5.3.2). Non-lytic viruses go thus undetected in the daily work of algal culture collections. This becomes problematic when algal strains are to be identified on the basis of their genetic signatures (Chapter 2); additional AFLP fragments will be generated by viral DNA. Two otherwise identical strains may result in two different genotypes if one is infected and the other is not. The aim of this study was therefore to assess the influence of viral contamination on AFLP banding patterns. Theoretically, a viral genome with up to $510 \mathrm{kbp}$ should lead to a small number of additional fragments in an alga with a genome size of ca. $38,800 \mathrm{kbp}$, the size for Chlorella vulgaris (Yamamoto et al. 2001). This theoretical assumption was tested in the present study. The objective was in addition to establish whether AFLP is an adequate method to detect a viral contamination by screening banding patterns for the presence of virus-specific patterns.

As test organisms, the lytic Chlorella virus PBCV-1 (genome size about $330 \mathrm{kbp}$ ) and its host Chlorella sp. NC64A (genome size about 40,000 kbp) were chosen. The first Chlorella viruses were discovered in the early 1980s in Chlorella-like algae (zoochlorella) symbiotic with Hydra viridis (Meints et al. 1981, Van Etten et al. 1981), and subsequently in Chlorella-like algae in Paramecium bursaria (e.g. Van Etten et al. 1983, Reisser et al. 1986). PBCV-1 stands for "Paramecium bursaria Chlorella virus" and is the prototype of a virus isolated from a culture of Chlorella sp. NC64A. This alga was isolated almost 45 years ago from Paramecium bursaria collected in the United States. When the algae were isolated from Paramecium, large dsDNA containing virus-like particles (VLPs) appeared within $24 \mathrm{~h}$ of isolation of the symbiotic Chlorella. The algae from $P$. bursaria can be also grown 
independent of Paramecium and the cultured Chlorella strains NC64A serve as hosts for many viruses. Zoochlorella growing symbiotically inside the paramecia did not contain VLPs. In an infected culture, the algae are killed by the lytic virus within eight hours. All information provided here about PBCV-1 and NC64A is based on Van Etten et al. (1991). AFLP analyses were performed on the pure virus culture, the pure Chlorella culture and infected Chlorella cultures for which the infection was stopped after one to 72 hours.

Analysis of multiple characters, e.g. morphology, physiology, biochemical and chemical components or random PCR, suggests that the Chlorella-like algae from Paramecium bursaria are closely related to Chlorella vulgaris Beijernick, but that there is not a single identical species for all local populations of paramecia worldwide (Reisser 1984, Kessler and Huss 1990, Takeda 1995, Kvitko et al. 2001). In the present study a close relationship with Chlorella vulgaris was supported by rDNA sequence analyses of Chlorella sp. NC64A. Comparison of the sequences obtained for Chlorella sp. NC64A with sequences available in public databases resulted in identical sequences for six zoochlorella strains isolated from Paramecium bursaria collected from various regions in Japan (Hoshina et al. 2004). Two of these isolates (OK1-ZK and F36-ZK) were axenic and thus were included in this study for AFLP analyses. In addition, an attempt was made to infect the Japanese strains with PCBV-1. 


\subsection{Materials and Methods}

\subsubsection{Investigated strains and infection experiments}

Strain Chlorella sp. NC64A was obtained from Prof. Gerhard Thiel, Institute of Botany, Darmstadt University of Technology. Infection experiments of this algal strain with PBCV-1 were performed by Dr. Mario Mehmel who is a member of the group of Prof. Thiel. Infection of strains was stopped after 1, 2, 3, 6, 24 and 72 h by freezing. Paramecian symbionts OK1-ZK and F36-ZK were kindly provided by Dr. Ryo Hoshina, Department of Biosciences and Biotechnology, Ritsumeikan University, Japan. Infection experiments were performed on these strains with PBCV-1 by Gisela Marxs from the group of Prof. Thiel.

\subsubsection{AFLP analyses}

DNA extraction and AFLP reactions were carried out as described for Chlorella vulgaris in Chapter 2 and the following three primer combinations were used in selective amplification: $E c o \mathrm{RI}+\mathrm{A} / M s e \mathrm{I}+\mathrm{C}, \quad E c o \mathrm{RI}+\mathrm{C} / M s e \mathrm{I}+\mathrm{C}$ and $E c o \mathrm{RI}+\mathrm{A} / M s e \mathrm{I}+\mathrm{G}$. All AFLP banding patterns were manually evaluated using the software GenScan from 100 to 500 bp.

\subsection{3 rDNA sequence analyses}

The 18S (Accession number DQ057341), ITS1, 5.8S and ITS2 regions (Acc. no. DQ057340) of the rDNA were amplified, sequenced, evaluated and submitted to BLASTn database searches as described in Chapter 2 for Chlorella vulgaris. 


\subsection{Results}

\subsubsection{AFLP analyses of pure and infected Chlorella cultures}

The pure culture of Chlorella sp. NC64A resulted in 197 AFLP fragments and the pure virus culture in 27 fragments for the three investigated primer combinations. Parts of the patterns of both strains are shown in Fig. 6.1A and B. If both patterns are displayed in one electropherogram, it is evident that the virus-specific fragments (black fragments in Fig. 6.1C) have different sizes than the alga-specific fragments (grey fragments in Fig. 6.1C). A total of 224 fragment positions were obtained and $13.1 \%$ of these fragment positions were virusspecific. AFLP patterns of the infection experiment stopped after one and two hours showed a mixed banding pattern of all virus-specific fragments and a reduced number of alga-specific fragments (Fig. 6.1D, E). No alga-specific fragments were obtained when the infection time was stopped after three hours (Fig. 6.1F). Longer infection times of six, 24 or 72 hours resulted in a pattern identical with that of the pure virus culture (Fig. 6.1G). The fragment intensities of the alga-specific fragments decreased with increased time after infection, whereas fragment intensities of the virus-specific fragments increased with increased time after infection.

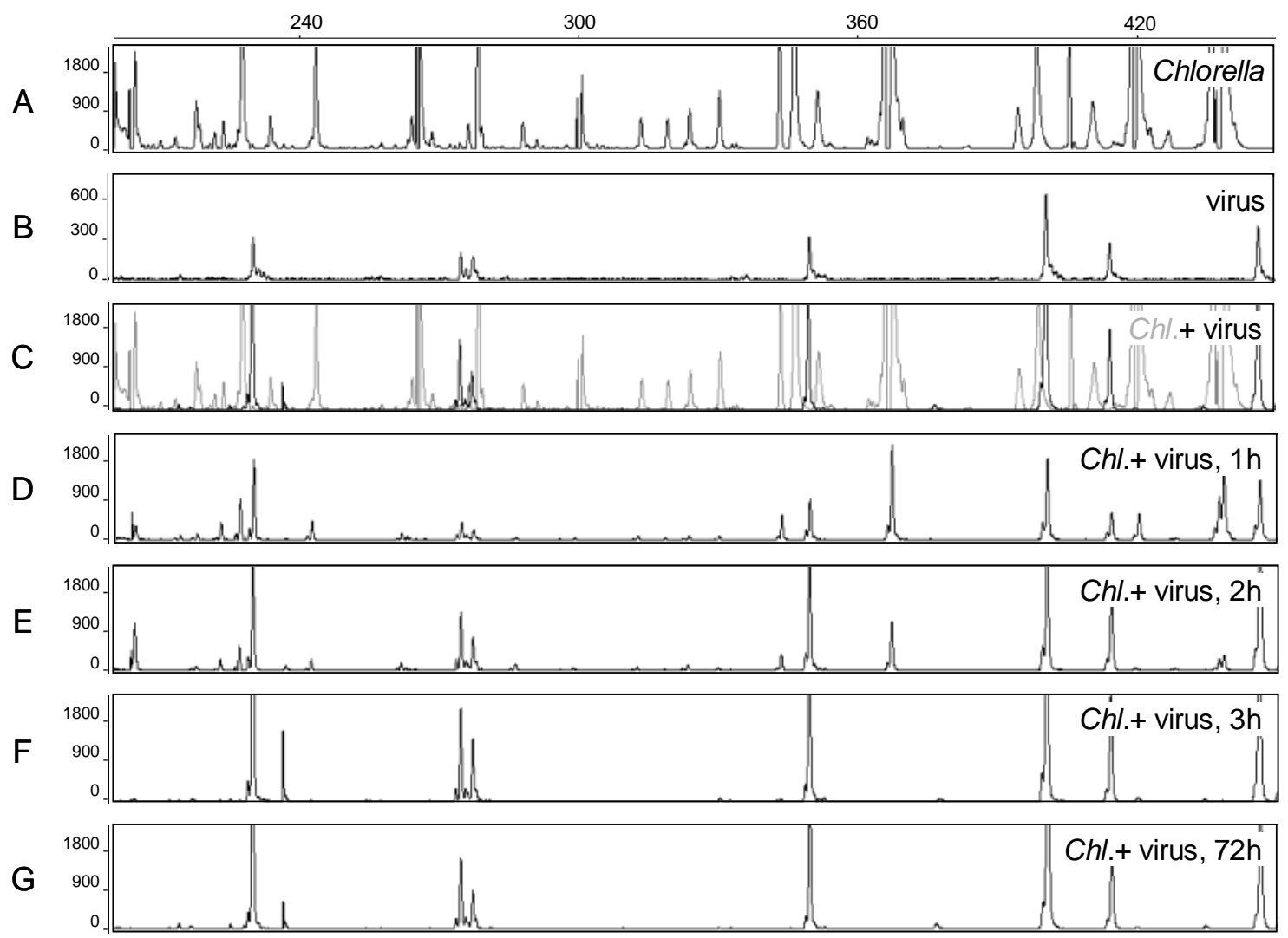

Fig. 6.1 AFLP banding patterns of Chlorella sp. NC64A pure culture (A), virus PBCV-1 pure culture (B), patterns of pure NC64A and PBCV-1 in one electropherogram and (C) of the infected cultures for which the infection was stopped after 1, 2, 3, or $72 \mathrm{~h}(\mathrm{D}-\mathrm{G})$. Electropherograms of EcoRI+G/Msel+C. Vertical scales, relative fluorescent units; horizontal scales, size of fragment in nucleotides. 


\subsubsection{Results of rDNA- and AFLP analyses and the infection experiment with the Japanese isolates}

The 18S rDNA gene of Chlorella sp. NC64A comprised 3250 bp including three introns of 327, 648 and 491 bp. The ITS1-5.8S-ITS2 rDNA comprised 275-159-242 bp. A blast search of the GenBank database revealed sequence identity for the 18S, ITS1, 5.8S and ITS2 regions with six strains of zoochlorella isolated from Paramecium bursaria collected in Japan (accession numbers AB162912-AB162917). NC64A also shared the three 18S rDNA introns at identical positions with the Japanese isolates. Two of the Japanese isolates were axenic and therefore investigated with AFLP.

The AFLP banding patterns of the two Japanese isolates, OK1-ZK and F36-ZK, were identical (Fig. 6.2). When compared with NC64A, eleven fragments (5.3\%) were found to be different. An example is shown in Fig. 6.2. The infection experiment of both Japanese strains using PBCV-1 was successful and resulted in the lysis of the strains.

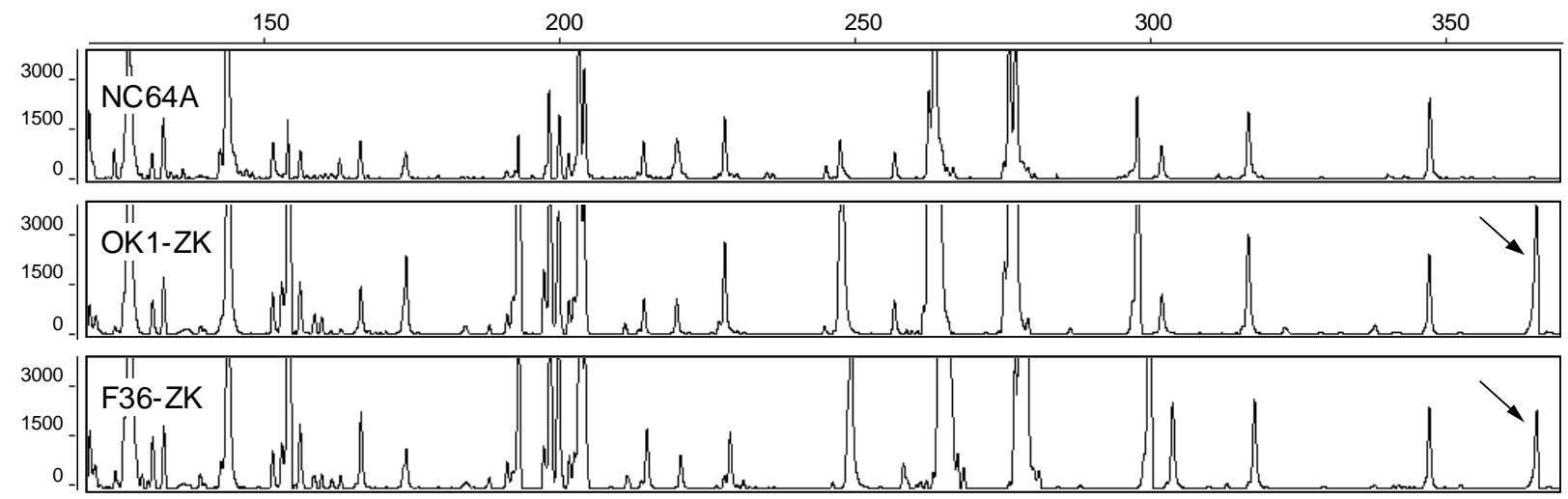

Fig. 6.2 AFLP banding patterns of Chlorella sp. NC64A and two Japanese algal isolates of Paramecium bursaria, OK1-ZK and F36-ZK. Arrows denote an additional fragment in the two Japanese isolates. Electropherograms of $E c o R I+A / M s e l+C$. Vertical scales, relative fluorescent units; horizontal scales, size of fragment in nucleotides. 


\subsection{Discussion}

\subsubsection{Detection of viral contaminations of algal strains using AFLP}

The detection of a viral contamination can be performed with AFLP by screening algal banding patterns for the presence of virus-specific patterns (black pattern in Fig. 6.1C). This AFLP-based detection method provides an interesting alternative to other available detection methods, because it is not restricted to axenic strains, does not require comparison with an uninfected culture and permits the detection of novel viruses.

\section{Detection of known and novel viruses}

The detection of known viruses is made possible by the fact that their AFLP fingerprints can be stored in a database and used for screening of banding patterns obtained of any algal culture. For this database, virus-specific fingerprints can be obtained by an AFLP reaction of a virus culture or can be calculated from all available complete genome sequences of viruses. In combination with a software-assisted screening mechanism, this database can represent a powerful detection method for viral infections not only in microalgae but also in any organism. Screening can be performed for both axenic (without bacteria or fungi) and non-axenic cultures. For investigations of non-axenic strains, a washing procedure is recommended before DNA extraction to reduce possible suppression in amplification of algaand virus-specific fragments by the DNA of the contaminating organisms (cf. Chapter 4).

For the detection of novel viruses with AFLP, only axenic cultures can be investigated and an uninfected culture is needed for comparison. If additional fragments in the range of around $13 \%$ (as found in the present study for PBCV-1) are found in the axenic putatively infected culture when compared to the uninfected culture, a viral infection is the only explanation for the additional fragments. Sequence analyses of excised additional fragments from a polyacrylamide gel can also help to determine the viral origin.

\subsubsection{Detection methods in algae}

Up to now, three different methods are used for viral detection in algae: visual inspection, bioassays and PCR-based methods (Lawrence 2005). For visual inspection, epifluorescence microscopy (EFM) or transmission electron microscopy (TEM) can be used, but examination of bacterialized cultures is problematic. In bioassays, characteristics of an uninfected culture (e.g. production of biomass) are compared with those of the potentially infected culture. On the molecular level, specific primers are available to amplify algal virus- 
specific gene fragments. Drawbacks of these three methods include the need for special equipment or an uninfected culture, and that primers are designed from known nucleic acid sequences, increasing the possibility that novel viruses may go undetected. The AFLP method therefore provides an interesting alternative solution for the detection of viral infections in microalgae.

\subsubsection{Detection of RNA containing viruses}

The drawback that no RNA-containing viruses can be detected with AFLP also applies to the other PCR-based detection methods. In the eukaryotic algae studied to date, three algal viruses have been isolated and characterized that contain RNA, while over 24 viruses are known that contain dsDNA. A detection method for DNA-containing viruses is therefore more important. However, the known RNA-containing viruses are all lytic and result in loss of the culture. Thus, if no reasons can be found why a culture was lost and AFLP- and PCRbased detection methods produced negative results, a visual inspection or a bioassay (see above) can be performed to establish whether a viral contamination is present.

\subsubsection{AFLP patterns show degradation of host DNA}

The disappearance of the genomic DNA of NC64A during PBCV-1 infection (Fig. 6.1) is consistent with some previous experiments (Van Etten et al. 1984, Burbank et al. 1990, McCluskey et al. 1992). In these experiments it was demonstrated that the host nuclear and chloroplast DNA is degraded, beginning at about one hour after infection and finishing in about three to four hours. This is in agreement with the disappearance of alga-specific fragments in AFLP patterns for infection times longer than three hours (Fig. 6.1F und G). It was assumed that the mitochondria would have to be reasonably stable to supply the tremendous energy requirement for the cells to replicate the virus (Van Etten, pers. comm.). However, it was not observed in this study that an alga-specific AFLP fragment possibly generated from mitochondrial DNA was still present after three hours of infection. This is due to the small genome size of the mitochondrium, which in Chlamydomonas reinhardtii (Accession number NC001638), for example, is only ca. $16 \mathrm{kbp}$. It was thus likely that no AFLP fragment could be generated from mitochondrial DNA. 


\subsubsection{Comparison of American and Japanese isolates to assess the host range of PBCV-1}

It was observed that AFLP is capable of detecting genetic differences between isolates that can be infected with the same virus (American and Japanese isolates, Fig. 6.2). From previous investigations it was known that viruses are limited with respect to the number of hosts they can infect, and are generally believed to infect hosts only within a single species (Lawrence 2005). This can be supported here for the American isolate and the two Japanese isolates which, based on the finding that their ITS rDNA sequences are identical, most likely represent the same species; all three Chlorella isolates are sensitive to PBCV-1. These findings support the assumption of Van Etten et al. (1991) that viral infection experiments provide a useful tool for classifying Chlorella species in the same way that bacteriophages are useful in bacterial classification. However, with AFLP a higher level of discrimination was achieved and differences were found between the American and the Japanese isolates. These results provide the basis for future analyses of a larger number of algae and virus isolates with the objective to correlate virus sensitivity with differences in AFLP patterns. Such a correlation is needed, because the few studies that have examined viruses of eukaryotic unicellular algae suggest that the host ranges of algal viruses are limited to within a species, but are complex at the strain level (Lawrence 2005). In microalgae, for example, it was shown that two viruses caused lysis of host strains only from the same geographical location (Tai et al. 2003). Cottrell and Suttle (1991), however, observed infection of host strains isolated from different geographical regions. In multicellular eukaryotic algae, by contrast, the situation is completely different, with available evidence suggesting that viruses can infect multiple species within a genus (Lawrence 2005). The present study shows that AFLP can provide additional data to better explain virus sensitivity patterns. 


\section{$7 \quad$ References}

Ahmadjian, V. 1967. A guide to the algae occurring as lichen symbionts: isolation, culture, cultural physiology and identification. Phycologia 6:127-60.

Álvarez, I. \& Wendel, J. F. 2003. Ribosomal ITS sequences and plant phylogenetic inference. Mol. Phylogenet. Evol. 29:417-34.

Arias, C. R., Welker, T. L., Shoemaker, C. A., Abernathy, J. W. \& Klesius, P. H. 2004. Genetic fingerprinting of Flavobacterium columnare isolates from cultured fish. $J$. Appl. Microbiol. 97:421-8.

Beaty, M. H. \& Parker, B. C. 1992. Cryopreservation of eukaryotic algae. Virgin. J. Sci. 43:403-10.

Behnke, A., Friedl, T., Chepurnov, V. A. \& Mann, D. G. 2004. Reproductive compatibility and rDNA sequence analyses in the Sellaphora pupula species complex (Bacillariophyta). J. Phycol. 40:193-208.

Beijernick, M. W. 1890. Culturversuche mit Zoochlorellen, Lichenengonidien und anderen niederen Algen. Bot. Z. 48:726-40.

Benson, E. E., 1990: Free radical damage in stored plant germplasm. Rome: International Board for Plant Genetic Resources, 128 pp.

Benson, E. E., 2004: Cryopreserving algal and plant diversity: historical perspectives and future challenges. In Fuller, B. J., Lane, N. \& Benson, E. E. [Eds.] Life in the frozen state. CRC Press LLC, Boca Raton.

Benson, E. E. \& Bremner, D. H., 2004: Oxidative stress in the frozen plant: a free radical point of view. In Fuller, B. J., Lane, N. \& Benson, E. E. [Eds.] Life in the frozen state. CRC Press LLC, Boca Raton, pp. 206-41.

Bodas, K., Brennig, C., Diller, K. R. \& Brand, J. J. 1995. Cryopreservation of blue-green and eukaryotic algae in the culture collection at the University of Texas in Austin. Cryo. Lett. 16:267-74.

Bonin, A., Bellemain, E., Bronken Eidesen, P., Pompanon, F., Brochmann, C. \& Taberlet, P. 2004. How to track and assess genotyping errors in population genetics studies. Mol. Ecol. 13:3261-73.

Boyer, S. L., Flechtner, V. R. \& Johansen, J. R. 2001. Is the 16S-23S rRNA internal transcribed spacer region a good tool for use in molecular systematics and population genetics? a case study in cyanobacteria. Mol. Biol. Evol. 18(6): 1057-1069.

Brand, J. J. \& Diller, K. R. 2004. Application and theory of algal cryopreservation. Nova Hedwigia 79:175-89.

Burbank, D. E., Shields, S. L., Schuster, A. M. \& Van Etten, J. L. 1990. 5-Azacytidineresistant mutants of Chlorella virus IL-3A. Virology 176:311-5.

Burke, S. A., Wright, J. D., Robinson, M. K., Bronk, B. V. \& Warren, R. L. 2004. Detection of molecular diversity in Bacillus atrophaeus by amplified fragment length polymorphism analysis. Appl. Environ. Microbiol. 70:2786-90.

Calcott, P. H. \& Gargett, A. M. 1981. Mutagenicity of freezing and thawing. FEMS Microbiology Letters 10:151-5.

Chavarriaga-Aguirre, P., Maya, M. M., Thome, J., Duque, C., Iglesias, C., Bonierbale, M. W., Kresovich, S. \& Kochert, G. 1999. Using microsatellites, isozymes and AFLPs to evaluate genetic diversity and redundancy in the cassava core collection and to assess the usefulness of DNA-based markers to maintain germplasm collections. Mol. Breed. 5:263-73.

Claes, H. 1954. Analyse der biochemischen Synthesekette für Carotinoide mit Hilfe von Chlorella-Mutanten. Z. Naturforschg. 9b:461-70.

Claes, H. 1956. Biosynthese von Carotinoiden bei Chlorella II. Tetrahydrolycopin und Lycopin. Z. Naturforschg. 11b:260-6. 
Claes, H. 1957. Biosynthese von Carotinoiden bei Chlorella III. Untersuchungen über die lichtabhängige Synthese von alpha- und beta- Carotin und Xanthophyllen bei der Chlorella Mutante 5/520. Z. Naturforschg. 12b:401-7.

Claes, H. 1958a. Biosynthese von Carotinoiden bei Chlorella IV. Die Carotinsynthese einer Chlorella Mutante bei anaerober Belichtung. Z. Naturforschg. 13b:222-4.

Claes, H. 1958b. Wie synthetisiert die Pflanze Carotinoide? Mitteilungen aus der MaxPlanck-Gesellschaft 5:276-84.

Claes, H. 1959. Biosynthese von Carotinoiden bei Chlorella V. Die Trennung von Licht- und Dunkelreaktion bei der lichtabhängigen Xanthophyllsynthese von Chlorella. Z. Naturforschg. 14b:4-7.

Claes, H. \& Nakayama, T. C. M. 1959. Isomerization of poly-cis-carotenes by chlorophyll in vivo and in vitro. Nature 183:1053.

Claes, H. 1960. Interaction between chlorophyll and carotenes with different chromophoric groups. Biochemical and Biophysical Research Communications 3:585-90.

Claes, H. 1961. Energieübertragung von angeregtem Chlorophyll auf $\mathrm{C}_{40}$-Polyene mit verschiedenen chromophoren Gruppen. Z. Naturforschg. 16b:445-54.

Claes, H. 1962. Untersuchungen an Chlorella-Mutanten: Die Energieübertragung von angeregtem Chlorophyll auf Carotinoide. Vorträge aus dem Gesamtgebiet der Botanik, herausgegeben von der Deutschen Botanischen Gesellschaft 1:109ff.

Claes, H. 1966. Maximal effectiveness of $670 \mathrm{~m} \mu$ in the light dependent carotinoid synthesis in Chlorella vulgaris. Photochemistry and Photobiology 5:515-21.

Coleman, A. W. \& Mai, J. C. 1997. Ribosomal DNA ITS-1 and ITS-2 sequence comparisons as a tool for predicting genetic relatedness. J. Mol. Evol. 45:168-77.

Coleman, A. W. 2000. The significance of a coincidence between evolutionary landmarks found in mating affinity and a DNA sequence. Protist 151:1-9.

Cottrell, M. T. \& Suttle, C. A. 1991. Wide-spread occurrence and clonal variation in viruses which cause lysis of a cosmopolitan, eukaryotic marine phytoplankter, Micromonas pusilla. Mar. Ecol. Prog. Ser. 79:1-9.

Crutchfield, A. L. M., Diller, K. R. \& Brand, J. J. 1999. Cryopreservation of Chlamydomonas reinhardtii (Chlorophyta). Eur. J. Phycol. 34:43-52.

Day, J. G. \& McLellan, M. R., 1995: Conservation of algae. In Grout, B. [Ed.] Genetic preservation of plant cells in vitro. Springer, Berlin, pp. 75-98.

Day, J. G., Watanabe, M. M., Morris, G. J., Fleck, R. A. \& McLellan, M. R. 1997. Long-term viability of preserved eukaryotic algae. J. Appl. Phycol. 9:121-7.

Day, J. G. 1998. Cryo-conservation of microalgae and cyanobacteria. Cryo Letters Supplement 1.

Day, J. G., Benson, E. E. \& Fleck, R. A. 1999. In vitro culture and conservation of microalgae: applications for aquaculture, biotechnology and environmental research. In Vitro Cell. Dev. Biol. Plant 35:127-36.

Day, J. G., Fleck, R. A. \& Benson, E. E. 2000. Cryopreservation-recalcitrance in microalgae: novel approaches to identify and avoid cryo-injury. J. Appl. Phycol. 12:369-77.

Day, J. G., Benson, E. E., Harding, K., Knowles, B., Idowu, M., Bremner, D. H., Watt, S., Santos, L., Friedl, T., Lorenz, M., Lukesova, A., Elster, J., Lukavsky, J., Herdman, M., Rippka, R., Hedoin, H. \& Hall, T., 2003: The use of cryopreservation to develop a European scientific and biotechnological resource: the COBRA project. In Lima, N. \& Smith, D. [Eds.] Biological Resource Centres and Use of Microbes. MUM, Minho, Portugal, pp. 307-20.

Day, J. G., Lukavsky, J., Friedl, T., Brand, J. J., Campbell, C. N., Lorenz, M. \& Elster, J. 2004. Pringsheim's living legacy: CCALA, CCAP, SAG and UTEX culture collections of algae. Nova Hedwigia 79:27-37. 
Day, J. G. \& Brand, J. J., 2005: Cryopreservation methods for maintaining cultures. In Andersen, R. A. [Ed.] Algal Culturing Techniques. Academic Press, New York, pp. 165-87.

De Bruin, A., Ibelings, B. W. \& van Donk, E. 2003. Molecular techniques in phytoplankton research: from allozyme electrophoresis to genomics. Hydrobiologia 491:47-63.

De Bruin, A., Ibelings, B. W., Rijkeboer, M., Brehm, M. \& van Donk, E. 2004. Genetic variation in Asterionella formosa (Bacillariophyceae): is it linked to frequent epidemics of host-specific parasitic fungi? J. Phycol. 40:823-30.

De Haan, L. R., Antonides, R., Belina, K. \& Ehlke, N. J. 2002. Peakmatcher: software for semi-automated fluorescence-based AFLP. Crop Sci. 42:1361-4.

De Varagas, C., Norris, R., Zaninetti, L., Gibb, S. W. \& Pawlowski, J. 1999. Molecular evidence of cryptic speciation in planktonic Foraminifers and their relation to oceanic provinces. Proc. Natl. Acad. Sci. USA 96:2864-8.

De-Bashan, L. E., Hernandez, J. P., Morey, T. \& Bashan, Y. 2004. Microalgae growthpromoting bacteria as "helpers" for microalgae: a novel approach for removing ammonium and phosphorus from municipal wastewater. Water Res. 38:466-74.

Després, L., Gielly, L., Redoutet, B. \& Taberlet, P. 2003. Using AFLP to resolve phylogenetic relationships in a morphologically diversified plant species complex when nuclear and chloroplast sequences fail to reveal variability. Mol. Phylogenet. Evol. 27:185-96.

Donaldson, S. L., Chopin, T. \& Saunders, G. W. 1998. Amplified fragment length polymorphism (AFLP) as a source of genetic markers for red algae. J. Appl. Phycol. 10:365-70.

Donaldson, S. L., Chopin, T. \& Saunders, G. W. 2000. An assessment of the AFLP method for investigating population structure in the red alga Chondrus crispus Stackhouse (Gigartinales, Florideophyceae ). J. Appl. Phycol. 12:25-35.

Dowling, T. E., Moritz, C., Palmer, J. D. \& Rieseberg, L. H., 1996: Nucleic acids III: analysis of fragments and restriction sites. In Hillis, D. M., Moritz, C. \& Mable, B. K. [Eds.] Molecular Systematics Second Edition. Sinauer Associates, Inc., Sunderland, pp. 249320.

Drake, J. W., Charlesworth, B., Charlesworth, D. \& Crow, J. F. 1998. Rates of spontaneous mutation. Genetics 148:1667-86.

Dyer, A. T. \& Leonard, K. J. 2000. Contamination, error, and nonspecific molecular tools. Phytopathology 90:565-7.

Erting, L., Daugbjerg, N. \& Moller Pedersen, P. 2004. Nucleotide diversity within and between four species of Laminaria (Phaeophyceae) analysed using partial LSU and ITS rDNA sequences and AFLP. Eur. J. Phycol. 39:24-256.

Fleck, R. A., Day, J. G., Rana, K. J. \& Benson, E. E. 1997. Visualisation of cryoinjury and freeze events in the coenocytic alga Vaucheria sessilis using cryomicroscopy. Cryo Letters 18:343-54.

Fleck, R. A., 1998: The assessment of cell damage and recovery in cryopreserved freshwater protists. PhD thesis, Universtiy Abertay Dundee, Dundee.

Fleck, R. A., Benson, E. E., Bremner, D. H. \& Day, J. G. 2000. Studies of free radicalmediated cryoinjury in the unicellular green alga Euglena gracilis using a nondestructive hydroxyl radical assay: a novel approach for developing protistan cryopreservation strategies. Free Radic Res 32:157-70.

Flores-Moya, A., Costas, E., Banares-Espana, E., Garcia-Villada, L., Altamirano, M. \& Lopez-Rodas, V. 2005. Adaptation of Spirogyra insignis (Chlorophyta) to an extreme natural environment (sulphureous waters) through preselective mutations. New Phytol. 166:655-61. 
Fott, B. \& Nováková, M., 1969: A monograph of the genus Chlorella. The fresh water species. In Fott, B. [Ed.] Studies in Phycology. Academia, Prague, pp. 10-74.

Friedl, T. 1995. Inferring taxonomic positions and testing genus level assignments in coccoid green lichen algae: a phylogenetic analysis of $18 \mathrm{~S}$ ribosomal RNA sequences from Dictyochloropsis reticulata and from members of the genus Myrmecia (Chlorophyta, Trebouxiophyceae cl. nov.). J. Phycol. 31:632-9.

Friedl, T. 1996. Evolution of the polyphyletic genus Pleurastrum (Chlorophyta): inferences from nuclear-encoded ribosomal DNA sequences and motile cell ultrastructure. Phycologia 35:456-69.

Friedl, T. \& O'Kelly, C. J. 2002. Phylogenetic relationships of green algae assigned to the genus Planophila (Chlorophyta): evidence from 18S rDNA sequence data and ultrastructure. Eur. J. Phycol. 3:373-84.

Gómez, P. I. \& González, M. A. 2004. Genetic variation among seven strains of Dunaliella salina (Chlorophyta) with industrial potential, based on RAPD banding patterns and on nuclear ITS rDNA sequences. Aquaculture 233:149-62.

González, M. A., Coleman, A. W., Gómez, P. I. \& Montoya, R. 2001. Phylogenetic relationship among various strains of Dunaliella (Chlorophyceae) based on nuclear ITS rDNA sequences. J. Phycol. 37:604-11.

Hall, T. A. 1999. BioEdit: a user-friendly biological sequence alignment editor and analysis program for Windows 95/98/NT. Nucl. Acids. Symp. Ser. 41:95-8.

Hansen, M., Kraft, T., Christiansson, M. \& Nilsson, N.-O. 1999. Evaluation of AFLP in Beta. Theor Appl Genet 98:845-52.

Hao, Y. J., Liu, Q.-L. \& Deng, X. X. 2001. Effect of cryopreservation on apple genetic resources at morphological, chromosomal, and molecular levels. Cryobiology 42:4653.

Hao, Y. J., You, C. X. \& Deng, X. X. 2002. Analysis of ploidy and the patterns of amplified fragment length polymorphism and methylation sensitive amplified polymorphism in strawberry plants recovered from cryopreservation. CryoLetters 23:37-46.

Harding, K. 2004. Genetic integrity of cryopreserved plant cells: a review. Cryo Letters 25:322.

Hauben, L., Vauterin, L., Moore, E. R. B., Hoste, B. \& Swings, J. 1999. Genomic diversity of the genus Stenotrophomonas. Int. J. Syst. Bacteriol. 49:1749-60.

Helliot, B., Madur, D., Dirlewanger, E. \& De Boucaud, M. T. 2002. Evaluation of genetic stability in cryopreserved Prunus. In Vitro Cell. Dev. Biol. Plant 38:493-500.

Hepperle, D., Hegewald, E. H. \& Krienitz, L. 2000. Phylogenetic position of the Oocystaceae (Chlorophyta). J. Phycol. 36:590-5.

Holm-Hansen, O. 1963. Viability of blue-green and green algae after freezing. Physiologia plantarum 16:530-40.

Hoshina, R., Kamako, S.-i. \& Imamura, N. 2004. Phylogenetic position of endosymbiotic green algae in Paramecium bursaria Ehrenberg from Japan. Plant Biology 6:447-53.

Huss, V. A. R., Scharpf, T. K. \& Kessler, E. 1989. Deoxyribonucleic acid reassociation in the taxonomy of the genus Chlorella. Arch. Microbiol. 152:512-4.

Huss, V. A. R. \& Sogin, M. L. 1990. Phylogenetic position of some Chlorella species within the Chlorococcales based upon complete small-subunit ribosomal RNA sequences. $J$. Mol. Evol. 31:432-42.

Huss, V. A. R., Frank, C., Hartmann, E. C., Hirmer, M., Kloboucek, A., Seidel, B. M., Wenzeler, P. \& Kessler, E. 1999. Biochemical taxonomy and molecular phylogeny of the genus Chlorella sensu lato (Chlorophyta) [Review]. J. Phycol. 35:587-98.

Iglesias-Rodriguez, M. D., Saez, A. G., Groben, R., Edwards, K. J., Batley, J., Medlin, L. K. \& Hayes, P. K. 2002. Polymorphic microsatellite loci in global populations of the marine coccolithophorid Emiliania huxleyi. Molecular Ecology Notes 2:495-7. 
Jarvis, E. E., Dunahay, T. G. \& Brown, L. M. 1992. DNA nucleoside composition and methylation in several species of microalgae. J. Phycol. 28:356-62.

Jin, E. S., Feth, B. \& Melis, A. 2002. A mutant of the green alga Dunaliella salina constitutively accumulates zeaxanthin under all growth conditions. Biotechnol. Bioeng. 81:115-24.

Jin, E. S. \& Melis, A. 2003. Microalgal biotechnology: carotenoid production by the green algae Dunaliella salina. Biotechnol. Bioproc. Eng. 8:331-7.

John, U., Groben, R., Beszteri, B. \& Medlin, L. 2004. Utility of amplified fragment length polymorphisms (AFLP) to analyse genetic structures within the Alexandrium tamarense species complex. Protist 155:169-79.

Jones, J., Edwards, A. W. F., Castaglione, S., Winfield, M. O., Sala, F., van de Wiel, C., Bredemeijer, K. J., Vosman, B., Matthes, M., Daly, A., Brettschneider, R., Bettini, P., Buiatti, M., Maestri, E., Malcevschi, A., Marmiroli, N., Aert, R., Volckaert, G., Rueda, J., Linacero, R., Vazquez, D. P. \& Karp, A. 1997. Reproducibility testing of RAPD, AFLP and SSR markers in plants by a network of European laboratories. Mol. Breed. 3:381-90.

Karlsson, J. O. M. \& Toner, M. 1996. Long term storage of tissues by cryopreservation: critical issues. Biomaterials 17:243-56.

Karsten, U., Friedl, T., Schumann, R., Hoyer, K. \& Lembcke, S. 2005. Mycosporine-like amino acids and phylogenies in green algae: Prasiola and its relatives from the Trebouxiophyceae (Chlorophyta). J. Phycol. in press.

Kessler, E. 1953. Über den Mechanismus der Nitratreduktion von Grünalgen II. Vergleichend-physiologische Untersuchungen. Arch. Mikrobiol. 19:438-57.

Kessler, E. 1976. Comparative physiology, biochemistry, and the taxonomy of Chlorella (Chlorophyceae). Plant Syst. Evol. 125:129-38.

Kessler, E. 1986. Limits of growth of five Chlorella species in the presence of toxic heavy metals. Arch. Hydrobiol. Suppl. 73:123-8.

Kessler, E. \& Huss, V. A. R. 1990. Biochemical taxonomy of symbiotic Chlorella strains from Paramecium and Acanthocystis. Bot. Acta 103:140-2.

Kessler, E., Kauer, G. \& Rahat, M. 1991. Excretion of sugars by Chlorella species capable and incapable of symbiosis with Hydra viridis. Bot. Acta 104:58-63.

Kooistra, W. H. C. F., Coppejans, G. G. \& Payri, C. 2002. Molecular systematics, historical ecology and phylogeography of Halimeda (Bryopsidales). Mol. Phylogenet. Evol. 24:121-38.

Koonjul, P. K., Brandt, W. F., Farrant, J. M. \& Lindsey, G. G. 1999. Inclusion of polyvinylpyrrolidone in the polymerase chain reaction reverses the inhibitory effects of polyphenolic contamination of RNA. Nucleic Acids Res. 27:915-6.

Krienitz, L., Hegewald, E. H., Hepperle, D., Huss, V. A. R., Rohr, T. \& Wolf, M. 2004. Phylogenetic relationship of Chlorella and Parachlorella gen. nov. (Chlorophyta, Trebouxiophyceae). Phycologia 43:529-42.

Kvitko, K. V., Migunova, A. V., Karelov, D. V. \& Prokosheva, M. J. 2001. Molecular taxonomy of virus-sensitive Chlorella sp.-symbionts of Paramecium bursaria. Protistology 2:96-104.

Lam, P. K. S., Wut, P. F., Chan, A. C. W. \& Wu, R. S. S. 1999. Individual and combined effects of cadmium and copper on the growth response of Chlorella vulgaris. Environ. Toxicol. 14:347-53.

Lawrence, C. W. 2002. Classical mutagenesis techniques. Methods Enzymol. 350:189-99.

Lawrence, J., 2005: Viral contamination of algal cultures. In Andersen, R. A. [Ed.] Algal Culturing Techniques. Academic Press, New York.

Lewis, L. A. \& Flechtner, V. R. 2004. Cryptic species of Scenedesmus (Chlorophyta) from desert soil communities of Western North America. J. Phycol. 40:1127-37. 
Liu, Y., Wang, X. \& Liu, L. 2004. Analysis of genetic variation in surviving apple shoots following cryopreservation by vitrification. Plant Science 166:677-85.

Lorenz, M., Friedl, T. \& Day, J. G., 2005: Perpetual maintenance of actively metabolizing microalgal cultures. In Andersen, R. A. [Ed.] Algal Culturing Techniques. Academic Press, New York, pp. 145-56.

Lyons-Weiler, J. \& Milinkovitch, M. C. 1997. A phylogenetic approach to the problem of differential lineage sorting. Mol. Bio. Evol. 14:968-75.

Mallick, N. 2002. Biotechnological potential of immobilized algae for wastewater N, P and metal removal: A review [Review]. BioMetals 15:377-90.

Mallick, N. 2003. Biotechnological potential of Chlorella vulgaris for accumulation of $\mathrm{Cu}$ and $\mathrm{Ni}$ from single and binary metal solutions. World J. Microbiol. Biotechnol. 19:695-701.

Manhart, J. R. \& McCourt, R. M. 1992. Molecular data and species concepts in the algae. $J$. Phycol. 28:730-7.

Mannschreck, B., Fink, T. \& Melzer, A. 2002. Biosystematics of selected Chara species (Charophyta) using amplified fragment lengths polymorphism (AFLP). Phycologia 41:657-66.

McCluskey, K., Graves, M. V., Mills, D. \& Meints, R. H. 1992. Replication of Chlorella virus PBCV-1 and host karyotype determination studied with pulsed-field gel electrophoresis. J. Phycol. 28:846-50.

McLenachan, P. A., Stöckler, K., Winkworth, R. C., McBreen, K., Zauner, S. \& Lockhart, P. J. 2000. Markers derived from amplified fragment length polymorphism gels for plant ecology and evolution studies. Mol. Ecol. 9:1899-903.

Meints, R. H., Van Etten, J. L., Kuczmarski, D., Lee, K. \& Ang, B. 1981. Viral infection of the symbiotic Chlorella-like alga present in Hydra viridis. Virology 113:698-703.

Misof, B., Rickert, A. M., Buckley, T. R., Fleck, G. \& Sauer, K. P. 2001. Phylogenetic signal and its decay in mitochondrial SSU and LSU rRNA gene fragments of Anisoptera. Mol. Bio. Evol. 18:27-37.

Mollenhauer, D. 2004. Historical aspects of culturing microalgae in Central Europe and the impact of Ernst Georg Pringsheim, a pioneer in algal culture collections. Nova Hedwigia 79:1-26.

Morris, G. J. 1978. Cryopreservation of 250 strains of Chlorococcales by the method of twostep cooling. Br. Phycol. J. 13:15-24.

Morris, G. J. \& Canning, C. E. 1978. The cryopreservation of Euglena gracilis. Journal of General Microbiology 108:27-31.

Mueller, U. G. \& LaReesa, W., L. 1999. Review AFLP genotyping and fingerprinting. Trends in Ecology and Evolution 14:389-94.

Murphy, N. E. \& Schaffelke, B. 2003. Use of amplified fragment length polymorphism (AFLP) as a new tool to explore the invasive green alga Caulerpa taxifolia in Australia. Mar. Ecol. Prog. Ser. 246:307-10.

Nei, M. \& Li, W. H. 1979. Mathematical model for studying genetic variation in terms of restriction endonucleases. Proc. Natl. Acad. Sci. USA 76:5269-73.

Pearson, B. M., Jackman, P. J. H., Painting, K. A. \& Morris, G. J. 1990. Stability of genetically manipulated yeast under different cryopreservation regimes. Cryo Letters 11:205-10.

Pelser, P. B., Gravendeel, B. \& van der Meijden, R. 2003. Phylogeny reconstruction in the gap between too little and too much divergence: the closest relatives of Senecio jacobaea (Asteraceae) according to DNA sequences and AFLPs. Mol. Phylogenet. Evol. 29:613-28. 
Penner, G. A., Bush, A., Wise, R., Kim, W., Domier, L., Kasha, K., Laroche, A., Scoles, G., Molnar, S. J. \& Fedak, G. 1993. Reproducibility of random amplified polymorphic DNA (RAPD) analysis among laboratories. PCR Methods Appl 2:341-5.

Pirson, A. 1937. Ernährungs- und stoffwechselphysiologische Untersuchungen an Fontinalis und Chlorella. Z. Bot. 31:193-267.

Pirson, A. 1994. Sixty years in algal physiology and photosynthesis. Photosyn. Res. 40:20921.

Preisig, H. R. \& Andersen, R. A., 2005: Historical review of algal culturing techniques. In Andersen, R. A. [Ed.] Algal Culturing Techniques. Academic Press, New York, pp. 112.

Pröschold, T., Marin, B., Schlosser, U. G. \& Melkonian, M. 2001. Molecular phylogeny and taxonomic revision of Chlamydomonas (Chlorophyta). I. Emendation of Chlamydomonas Ehrenberg and Chloromonas Gobi, and description of Oogamochlamys gen. nov. and Lobochlamys gen. nov. Protist 152:265-300.

Pulz, O. 2001. Photobioreactors: production systems for phototrophic microorganisms [Review]. Appl. Microbiol. Biotechnol. 57:287-93.

Reisser, W. 1984. The taxonomy of green algae endosymbiotic in Cilliates and Sponges. $\mathrm{Br}$. Phycol. J. 19:309-18.

Reisser, W., Becker, B. \& Klein, T. 1986. Studies on ultrastructure and host range of a Chlorella attacking virus. Protoplasma 135:162-5.

Rosenberg, N. A. 2002. The probability of topological concordance of gene trees and species trees. Theor. Popul. Biol. 61:225-47.

Sauer, K., 2004: DNA-Fragmentanalysen zur genetischen Charakterisierung von Cyanobakterien-Kulturen und Kryokonservierung zu ihrer Erhaltung, Experimental Phycology and Culture Collection of Algae, University of Göttingen, Göttingen.

Savelkoul, P. H. M., Aarts, H. J. M., De Haas, J., Dijkshoorn, B., Duim, B., Otsen, M., Rademaker, W., Schouls, L. \& Lenstra, J. A. 1999. Minireview amplified fragment length polymorphism analysis: the state of an art. J. Clin. Microbiol. 37:3083-91.

Schaeffer, T. N., Smith, G. J. \& Foster, M. S. 2002. Genetic differences between two growthforms of Lithophyllum margaritae (Rhodophyta) in Baja California Sur, Mexico. J. Phycol. 38:1090-8.

Schlösser, U. G. 1994. SAG - Sammlung von Algenkulturen at the University of Göttingen. Catalogue of strains 1994. Botanica Acta 107:113-86.

Schwarze, P. \& Frandsen, N. O. 1960. Herstellung von Chlorella-Farbmutanten mit Hilfe von radioaktiven Isotopen. Die Naturwissenschaften 47:47.

Schwarze, P. 1965a. Untersuchungen über die Förderung der Chlorophyllbildung durch Arginin und Glycin bei einer chlorophyllarmen Mutante von Chlorella vulgaris. Z. Bot. 52:510-25.

Schwarze, P. 1965b. Über das Vorkommen einer flüchtigen Säure in Nährlösungen von Chlorella-Kulturen. Z. Bot. 52:526-31.

Scragg, A. H. \& Bonnett, C. 2002. Inhibition of microalgal growth by silver nitrate. Biotechnol. Lett. 24:169-72.

Scragg, A. H., Morrison, J. \& Shales, S. W. 2003. The use of a fuel containing Chlorella vulgaris in a diesel engine. Enzyme Microb. Technol. 33:884-9.

Sneath, P. H. \& Sokal, R. R., 1973: Numerical Taxonomy: The Principles and Practice of Numerical Classification. San Francisco, CA.

Swofford, D. L. 2001. PAUP*. Phylogenetic analysis using Parsimony (*and other methods). Version 4.0b8. Sinauer, Sunderland MA.

Tai, V., Lawrence, J., Lang, A. S., Chan, A. M., Culley, A. I. \& Suttle, C. A. 2003. Characterization of HaRNAV, a single-stranded RNA virus causing lysis of Heterosigma akashiwo (Raphidophyceae). J. Phycol. 39:343-52. 
Takeda, H. 1988. Classification of Chlorella strains by cell wall sugar composition. Phytochemistry 27:3823-6.

Takeda, H. 1991. Sugar composition of the cell wall and the taxonomy of Chlorella (Chlorophyceae). J. Phycol. 27:224-32.

Takeda, H. 1993. Taxonomical assignment of Chlorococcal algae from their cell wall composition. Phytochemistry 34:1053-5.

Takeda, H. 1995. Cell wall composition and taxonomy of symbiotic Chlorella from Paramecium and Acanthocystis. Phytochemistry 40:457-9.

Tam, N. F. Y., Lau, P. S. \& Wong, Y. S. 1994. Wastewater inorganic N and P removal by immobilized Chlorella vulgaris. Water Sci. Technol. 30:369-74.

Tam, N. F. Y., Wong, Y. S. \& Simpson, C. G. 1998. Repeated removal of copper by alginate beads and the enhancement by microalgae. Biotechnol. Techn. 12:187-90.

Tamiya, H., Iwamura, T., Shibata, K., Hase, E. \& Nihei, T. 1953. Correlation between photosynthesis and light-independent metabolism in the growth of Chlorella. Biochim. Biophys. Acta 12:23-40.

Taylor, J. W., Jacobson, D. J., Kroken, S., Kasuga, T., Geiser, D. M., Hibbett, D. S. \& Fisher, M. C. 2000. Phylogenetic species recognition and species concepts in fungi. Fungal Genet. Biol. 31:21-32.

Taylor, J. W. \& Fisher, M. C. 2003. Fungal multilocus sequence typing - it's not just for bacteria. Curr. Opin. Microbiol. 6:351-6.

Taylor, R. \& Fletcher, R. L. 1999. Cryopreservation of eukaryotic algae - a review of methodologies. J. Appl. Phycol. 10:481-501.

Turner, S. e. a. 2001. Genetic fidelity and viability of Anigozanthos viridis following tissue culture, cold storage and cryopreservation. Plant Science 161:1099-106.

Van Etten, J. L., Meints, R. H., Burbank, D. E., Kuczmarski, D., Cupples, D. A. \& Lane, L. C. 1981. Isolation and characterization of a virus from the intracellular green alga symbiotic with Hydra viridis. Virology 113:704-11.

Van Etten, J. L., Burbank, D. E., Kuczmarski, D. \& Meints, R. H. 1983. Virus infection of culturable Chlorella-like algae and development of a plaque assay. Science 219:994-6.

Van Etten, J. L., Burbank, D. E., Joshi, J. \& Meints, R. H. 1984. DNA synthesis in a Chlorella-like alga following infection with the virus PBCV-1. Virology 134:443-9.

Van Etten, J. L., Lane, L. C. \& Meints, R. H. 1991. Viruses and viruslike particles of eukaryotic algae. Microbiol. Rev. 55:586-620.

Vannini, G. L. \& Poli, F. 1983. Binucleation and abnormal chromosome distribution in Euglena gracilis cells treated with dimethyl sulfoxide. Protoplasma 114:62-6.

Vos, P., Hogers, R., Bleeker, M., Reijans, M., van de Lee, T., Hornes, M. \& Frijters, A. 1995. AFLP: a new technique for DNA fingerprinting. Nucleic Acids Res. 23:4407-14.

Wakasugi, T., Nagai, T., Kapoor, M., Sugita, M., Ito, M., Ito, S., Tsudzuki, J., Nakashima, K., Tsudzuki, T., Suzuki, Y., Hamada, A., Ohta, T., Inamura, A., Yoshinaga, K. \& Sugiura, M. 1997. Complete nucleotide sequence of the chloroplast genome from the green algae Chlorella vulgaris: the existence of genes possibly involved in chloroplast division. Proc. Natl. Acad. Sci. USA 94:5967-72.

Warburg, O. 1919. Über die Geschwindigkeit der Kohlensäurezersetzung in lebenden Zellen. Biochem. Z. 100:230-70.

Werner, R., Olschewski, J. \& Mergenhagen, D. 2001. Identification and cloning of amplified fragment length polymorphism markers linked to the mating type locus of Chlamydomonas reinhardii (Chlorophyta). J. Phycol. 37:427-3.

Wilkinson, T., Wetten, A., Prychid, C. \& Fay, M. F. 2003. Suitability of cryopreservation for the long-term storage of rare and endangered plant species: a case history for Cosmos atrosanguineus. Annals of Botany 91:1-10. 
Wong, J. P. K., Wong, Y. S. \& Tam, N. F. Y. 2000. Nickel bioabsorption by two Chlorella species, C. vulgaris (a commercial species) and C. miniata (a local isolate). Bioresour. Technol. 73:133-7.

Yamamoto, M., Nozaki, H. \& Kawano, S. 2001. Evolutionary relationships among multiple modes of cell division in the genus Nannochloris (Chlorophyta) revealed by genome size, actin gene multiplicity, and phylogeny. J. Phycol. 37:106-20.

Zhang, F., Kabeya, H., Kitagawa, R., Hirotsu, T., Yamashita, M. \& Otsuki, T. 2000. An exploratory research of PVC-Chlorella composite material (PCCM) as effective utilization of Chlorella biologically fixing $\mathrm{CO}_{2}$. J. Materials Sci. 35:2603-9. 


\section{Danksagung}

Dass ich an jedem Morgen gerne ins Labor komme, liegt an der ganz großartigen EPSAGArbeitsgruppe. Deshalb möchte ich „Dankeschön” sagen an:

Thomas Friedl, der mich mit seiner Begeisterung für mein Thema immer wieder angesteckt hat, mich darin unterstützt hat, an Tagungen teilzunehmen und einige Wochen in anderen Laboren arbeiten zu können. Ich danke für die umfangreiche Betreuung meiner Arbeit, für immer neue Denkanstöße, Diskussionen und die Hilfe meine Arbeit in die vorliegende Form zu bringen. Herzlichen Dank!

Maike Lorenz, die meine Arbeit mit vielen Anregungen und konstruktiver Kritik unterstützt hat und immer Zeit für mich hatte. Dankeschön für die vielen guten Ideen und Diskussionen!

Elke Zufall-Roth, Hella Timmermann, Ilse Kunkel, Marlis Heinemann, Gaby Schauermann, Ruth Pilot und Birgit Olberg für die tolle Zusammenarbeit im Labor, fröhliche Frühstückspausen und vor allem dafür, dass ich immer auf Ihre Unterstützung zählen konnte.

Den „Mädels“ für die tolle Zeit im Diplomandenzimmer und/oder für viel schöne (Koch-) Abende: Anke Behnke (Danke auch fürs Korrigieren!), Maike Lorenz (ein zweites Dankeschön!), Michaela Kitschke, Jana Fredersdorf, Imke Lang, Viviane Kipp, Kerstin Holst, Kerstin Pick, Maria Siegesmund (Danke auch fürs Korrigieren!) und Eva Völler sowie Kristin Sauer und Steffi Gäbler (Danke auch für die Unterstützung im Labor!). Und natürlich auch Christian Opayi Mudimu, den ich ja nicht unter „Mädels“ nennen konnte.

Dominik Hepperle für seine Hilfe bei den AFLP-Analysen und für seine konstruktive Kritik an meiner Arbeit und meinen Vorträgen.

Dierk Mende für die unschätzbare Hilfe in Computerfragen.

Prof. Dr. Uwe G. Schlösser für die Bereitstellung der umfangreichen Informationen über die SAG-Stämme, die in der vorliegenden Arbeit untersucht wurden.

Aber auch außerhalb unserer Arbeitsgruppe bin ich auf Unterstützung gestoßen.

Deshalb geht ein weiteres „Dankeschön” an:

Prof. Dr. Ivo Feußner für die freundliche Übernahme des Korreferats.

Allen Partnern aus dem EU-Projekt „COBRA“ (QLRI-CT-2001-01645), vor allem John G. Day, Erica B. Benson und Keith Harding für ein erfolgreiches Projekt. Es hat mir viel Freude gemacht, ein Teil davon zu sein. Danke auch für die Finanzierung durch dieses Projekt.

Thomas Pröschold für viele anregende Diskussionen über Chlamy, AFLP und Co.

Prof. Dr. Gerhard Thiel, Dr. Mario Mehmel und Gisela Marx (Bot. Institut, TU Darmstadt) für die Durchführung der Vireninfektionsversuche sowie die Bereitstellung der Algen- und Virenkulturen. Prof. Dr. Van Etten für den sehr hilfreichen Email-Kontakt bezüglich PBCV-1.

Prof. Dr. João Varel (CCMar, Portugal), Dr. Jürgen Polle (DCCBC, USA) und Dr. Ryo Hoshina (Ritsumeikan Universität, Japan) für die Bereitstellung von Algenkulturen.

Beate Mannschreck für die Einweisung in die AFLP-Technik.

Mein ganz besonderer Dank geht an meinen Mann Torsten und meine Eltern sowie an Eicke, Karin und Christopher. Eure Zuversicht hat mir sehr geholfen! 


\section{Lebenslauf}

Julia Müller

geboren am 14. Dezember 1976

in Northeim

\section{Doktorarbeit}

seit 01.2002

in der Abteilung Experimentelle Phykologie und Sammlung von Algenkulturen,

Albrecht-von-Haller-Institut für Pflanzenwissenschaften, Biologische Fakultät,

Georg-August-Universität Göttingen

\section{Studium Lehramt Biologie und Chemie}

$10.1996-12.2001$

$12.2000-07.2001$

Schulbildung

$08.1993-05.1996$ für Gymnasien, an der Universität Göttingen

Abschluss: Erstes Staatsexamen

Staatsexamensarbeit („Methoden zur Erfassung der genetischen Diversität mikroskopischer Grünalgen“) in Biologie in der Abteilung Experimentelle Phykologie und Sammlung von Algenkulturen, Albrecht-von-Haller-Institut für Pflanzenwissenschaften, Biologische Fakultät, Georg-August-Universität Göttingen bei Prof. Dr. Thomas Friedl

Fachgymnasium Wirtschaft, Northeim

Abschluss: Abitur 